\title{
Multiple codings of self-similar sets with overlaps
}

\author{
Karma Dajani $^{a}$, Kan Jiang ${ }^{b, *}$, Derong Kong ${ }^{c}$, Wenxia Li $^{\mathrm{d}}$, \\ Lifeng $\mathrm{Xi}^{\mathrm{b}}$ \\ a Department of Mathematics, Utrecht University, Budapestlaan 6, P.O. Box \\ 80.000, 3508 TA Utrecht, the Netherlands \\ b Department of Mathematics, Ningbo University, Ningbo, Zhejiang, People's \\ Republic of China \\ c College of Mathematics and Statistics, Chongqing University, 401331, Chongqing, \\ People's Republic of China \\ d School of Mathematical Sciences, Shanghai Key Laboratory of PMMP, East \\ China Normal University, Shanghai 200062, People's Republic of China
}

\section{A R T I C L E I N F O}

\section{Article history:}

Received 21 April 2020

Received in revised form 24

November 2020

Accepted 1 December 2020

Available online 9 December 2020

\section{$M S C$ :}

primary 11A63

secondary 37B10, 28A78, 10K50, $11 \mathrm{~K} 55$

\section{Keywords:}

Unique expansion

Multiple expansions

Countable expansions

Hausdorff dimension
A B S T R A C T

In this paper we consider a general class $\mathcal{E}$ of self-similar sets with complete overlaps. Given a self-similar iterated function system $\Phi=\left(E,\left\{f_{i}\right\}_{i=1}^{m}\right) \in \mathcal{E}$ on the real line, for each point $x \in E$ we can find a sequence $\left(i_{k}\right)=i_{1} i_{2} \ldots \in\{1, \ldots, m\}^{\mathbb{N}}$, called a coding of $x$, such that

$$
x=\lim _{n \rightarrow \infty} f_{i_{1}} \circ f_{i_{2}} \circ \cdots \circ f_{i_{n}}(0) .
$$

For $k=1,2, \ldots, \aleph_{0}$ or $2^{\aleph_{0}}$ we investigate the subset $\mathcal{U}_{k}(\Phi)$ which consists of all $x \in E$ having precisely $k$ different codings. Among several equivalent characterizations we show that $\mathcal{U}_{1}(\Phi)$ is closed if and only if $\mathcal{U}_{\aleph_{0}}(\Phi)$ is an empty set. Furthermore, we give explicit formulae for the Hausdorff dimension of $\mathcal{U}_{k}(\Phi)$, and show that the corresponding Hausdorff measure of $\mathcal{U}_{k}(\Phi)$ is always infinite for any $k \geq 2$. Finally, we explicitly calculate the local

\footnotetext{
* Corresponding author.

E-mail addresses: k.dajani1@uu.nl (K. Dajani), kanjiangbunnik@yahoo.com (K. Jiang), derongkong@126.com (D. Kong), wxli@math.ecnu.edu.cn (W. Li), xilifengningbo@yahoo.com (L. Xi).
} 
dimension of the self-similar measure at each point in $\mathcal{U}_{k}(\Phi)$ and $\mathcal{U}_{\aleph_{0}}(\Phi)$.

\section{Contents}

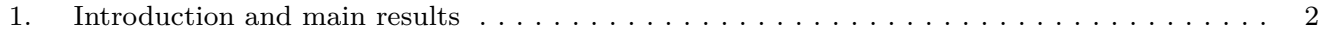

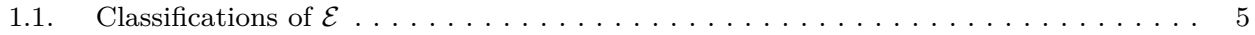

1.2. Hausdorff dimension and Hausdorff measure of $\mathcal{U}_{k}(E) \ldots \ldots \ldots \ldots$

1.3. Local dimension of a self-similar measure in $\mathcal{U}_{k}(\Phi)$ and $\mathcal{U}_{\aleph_{0}}(\Phi) \ldots \ldots \ldots$

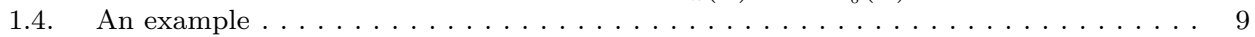

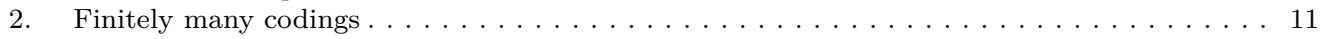

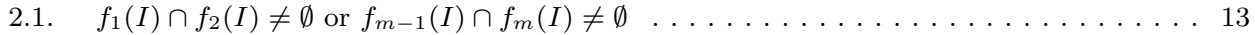

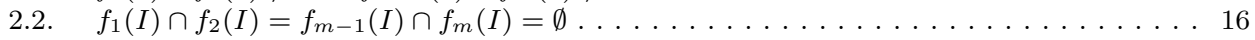

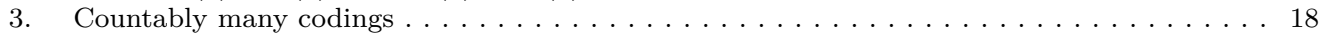

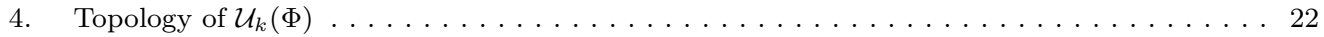

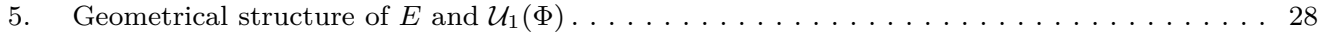

5.1. Geometrical structure of $\mathcal{U}_{1}(\Phi) \ldots \ldots \ldots \ldots \ldots \ldots \ldots$

5.2. Geometrical structure of $E \ldots \ldots \ldots \ldots \ldots \ldots \ldots$

6. Hausdorff dimensions and Hausdorff measures of $E$ and $\mathcal{U}_{k}(\Phi) \ldots \ldots \ldots$

6.1. Hausdorff dimensions of $E$ and $\mathcal{U}_{k}(\Phi) \ldots \ldots \ldots \ldots \ldots \ldots$

6.2. Hausdorff measure of $\mathcal{U}_{k}(\Phi) \ldots \ldots \ldots \ldots \ldots \ldots \ldots \ldots \ldots \ldots$

7. Local dimension of self-similar measure . . . . . . . . . . . . . . . . . . . . . . . . . . . 39

7.1. Local dimension of $\mu_{\mathrm{p}}$ at points in $\mathcal{U}_{k}(\Phi) \ldots \ldots \ldots \ldots \ldots \ldots$

7.2. Local dimension of $\mu_{\mathrm{p}}$ at points in $\mathcal{U}_{\aleph_{0}}(\Phi) \ldots \ldots \ldots \ldots \ldots \ldots$

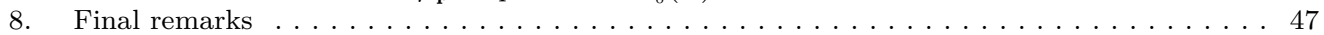

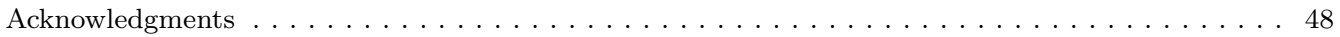

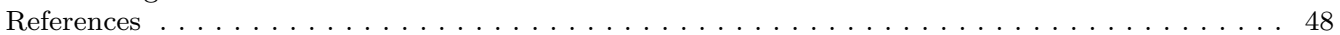

\section{Introduction and main results}

Given $\beta \in(1,2]$, for each $x \in I_{\beta}:=[0,1 /(\beta-1)]$ there exists a sequence $\left(d_{i}\right)$ of zeros and ones such that

$$
x=\sum_{i=1}^{\infty} \frac{d_{i}}{\beta^{i}},
$$

and the sequence $\left(d_{i}\right)$ is called a $\beta$-expansion of $x$. Non-integer base expansions of reals, as a natural extension of dyadic expansions, were pioneered by Rényi [30] and Parry [28]. In 1990s Erdős et al. [10-12] discovered that for each $k=1,2, \cdots$ or $\aleph_{0}$ there exist a base $\beta \in(1,2)$ and a number $x \in I_{\beta}$ such that $x$ has exactly $k$ different $\beta$-expansions. This turns out to be very different from the dyadic expansions, where each $x \in I_{2}$ has a unique dyadic expansion excluding countably many exceptions. After the exciting discovery of Erdős and his collaborators there was a great interest in the study of non-integer base expansions. By using ergodic theorem Sidorov showed in [31] that for $\beta \in(1,2)$ Lebesgue almost every $x \in I_{\beta}$ has a continuum of $\beta$-expansions (see also, [3]). In the past 30 years 
there has been a great progress on non-integer base expansions, especially on unique $\beta$-expansions (see example, $[1,6,15,22]$ ). For finite $\beta$-expansions, very little is known (see $[2,21,32])$. This motivates us to study the multiple expansions (codings) in a fractal setting. For more information on non-integer base expansions we refer the reader to the survey paper [20] and the survey chapter [7].

In this paper we consider multiple expansions for self-similar sets with overlaps. For $1 \leq i \leq m$ let $f_{i}(\cdot)$ be a similitude on $\mathbb{R}$ defined by

$$
f_{i}(x)=r_{i} x+b_{i}
$$

where $r_{i} \in(0,1)$ and $b_{i} \in \mathbb{R}$. Then there exists a unique non-empty compact set $E \subset \mathbb{R}$ satisfying (cf. [17])

$$
E=\bigcup_{i=1}^{m} f_{i}(E) .
$$

In this case, the couple $\Phi=\left(E,\left\{f_{i}\right\}_{i=1}^{m}\right)$ is called a self-similar iterated function system (SIFS), and the compact set $E$ is called a self-similar set generated by $\left\{f_{i}\right\}_{i=1}^{m}$.

Now we introduce a class $\mathcal{E}$ of $\operatorname{SIFS} \Phi=\left(E,\left\{f_{i}\right\}_{i=1}^{m}\right)$ on $\mathbb{R}$ which will be our object throughout the paper. Denote by $I=[a, b]$ the convex hull of the self-similar set $E$. We say that $\Phi \in \mathcal{E}$ if it satisfies the following conditions (A)-(D).

(A) $a=f_{1}(a)<f_{2}(a)<\cdots<f_{m}(a)<f_{m}(b)=b$.

(B) $f_{i}(I) \cap f_{i+2}(I)=\emptyset$ for any $i \in\{1, \ldots, m-2\}$.

(C) There exist $i, j \in\{1, \cdots, m-1\}$ such that

$$
f_{i}(I) \cap f_{i+1}(I) \neq \emptyset \quad \text { and } \quad f_{j}(I) \cap f_{j+1}(I)=\emptyset .
$$

(D) If $f_{i}(I) \cap f_{i+1}(I) \neq \emptyset$, then there exist positive integers $u_{i}$ and $v_{i}$ such that

$$
f_{i}(I) \cap f_{i+1}(I)=f_{i m^{u_{i}}}(I)=f_{(i+1) 1^{v_{i}}}(I),
$$

where $f_{i_{1} \cdots i_{k}}(\cdot):=f_{i_{1}} \circ \cdots \circ f_{i_{k}}(\cdot)$ denotes the compositions of the maps $f_{i_{1}}, \ldots, f_{i_{k}}$.

The intervals $f_{i}(I), i=1, \cdots, m$ are called the basic intervals of $\Phi=\left(E,\left\{f_{i}\right\}_{i=1}^{m}\right)$. Then by Conditions (A)-(D) it follows that $m \geq 3$, and the basic intervals are located from left to right in the following way (see, e.g., Fig. 1). The most left one is $f_{1}(I)$, and the second one is $f_{2}(I)$, and the most right one is $f_{m}(I)$. Furthermore, there exist two neighboring basic intervals having a non-empty intersection, and there also exist two neighboring basic intervals having an empty intersection. But any three basic intervals must have an empty intersection. By Condition (D) it follows that any basic interval cannot be included in another basic interval, and the intersection of basic intervals cannot be a singleton. 

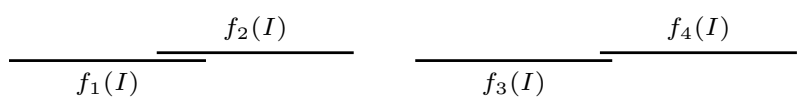

Fig. 1. The basic intervals $f_{1}(I), f_{2}(I), f_{3}(I)$ and $f_{4}(I)$ with $I=[0,1]$, and the maps $f_{i}, 1 \leq i \leq 4$ are defined as in (1.3) with $\beta=4, M=1, N=2$. Then $f_{1}(I) \cap f_{2}(I)=f_{14}(I)=f_{21}(I)$ and $f_{3}(\bar{I}) \cap \bar{f}_{4}(I)=$ $f_{344}(I)=f_{411}(I)$. So the $\operatorname{SIFS} \Phi=\left(E,\left\{f_{i}\right\}_{i=1}^{4}\right) \in \mathcal{E}$. Then $\operatorname{dim}_{H} \mathcal{U}_{k}(\Phi)=\operatorname{dim}_{H} \mathcal{U}_{1}(\Phi) \approx 0.0943436$ and $\operatorname{dim}_{H} \mathcal{U}_{2^{\aleph_{0}}}(\Phi)=\operatorname{dim}_{H} E \approx 0.934164$. See Example 1.4 for more explanations.

Here we mention that Condition (D) is always associated with the complete overlap condition (cf. [16]). This class of complete overlapping SIFSs has been studied by many people from different aspects. Ngai and Wang [26] calculated the Hausdorff dimension of the overlapping self-similar sets. Rao and Wen [29] considered the topology of a special class of overlapping self-similar sets $E_{\lambda}$ generated by $f_{1}(x)=\frac{x}{3}$, $f_{2}(x)=\frac{x+\lambda}{3}$ and $f_{3}(x)=\frac{x+2}{3}$, where $\lambda \in[0,1]$. In particular, they determined for which rational numbers $\lambda$ the self-similar set $E_{\lambda}$ contains interior points (Kenyon [19] proved a similar result). The generating IFSs for $E_{\lambda}$ was recently investigated by Dajani et al. [5]. Let $\Phi=\left(E,\left\{f_{i}\right\}_{i=1}^{m}\right) \in \mathcal{E}$. Then for any $x \in E$ there exists a sequence $\left(d_{i}\right)=d_{1} d_{2} \cdots \in\{1,2, \cdots, m\}^{\mathbb{N}}$ such that (cf. [13])

$$
x=\lim _{n \rightarrow \infty} f_{d_{1} \cdots d_{n}}(0)=: \pi\left(\left(d_{i}\right)\right) .
$$

The sequence $\left(d_{i}\right)$ is called a coding of $x$ with respect to the alphabet $\{1, \ldots, m\}$. We point out that a point $x \in E$ may have multiple codings by Conditions (C) and (D). For $k=1,2, \cdots, \aleph_{0}$ or $2^{\aleph_{0}}$ we set

$$
\mathcal{U}_{k}(\Phi):=\{x \in E: x \text { has exactly } k \text { different codings }\}
$$

Recently, the first four authors [4] considered a special candidate $\Phi_{0}=\left(E,\left\{f_{i}\right\}_{i=1}^{3}\right)$ of $\mathcal{E}$ which was first introduced by Ngai and Wang [26], where

$$
f_{1}(x)=\frac{x}{\beta}, \quad f_{2}(x)=\frac{x+1}{\beta}, \quad f_{3}(x)=\frac{x+\beta}{\beta}
$$

with $\beta>(3+\sqrt{5}) / 2$. Based on the characterization of $\mathcal{U}_{1}\left(\Phi_{0}\right)$ given by Zou et al. [33] they showed that the Hausdorff dimensions of $\mathcal{U}_{k}\left(\Phi_{0}\right)$ are the same for all integers $k \geq 1$.

Inspired by the work of [4] we investigate the multiple codings of the SIFSs in $\mathcal{E}$ from different aspects. (I) We classify the collection $\mathcal{E}$ via the multiple codings set $\mathcal{U}_{k}(\Phi)$, see Theorem 1; (II) We calculate in Theorem 2 the Hausdorff dimension of $\mathcal{U}_{k}(\Phi)$, and 
show that $\mathcal{U}_{k}(\Phi)$ has infinite Hausdorff measure for any $k \geq 2$ assuming $\mathcal{U}_{k}(\Phi) \neq \emptyset$; (III) We determine in Theorem 3 the local dimension of the self-similar measure at points in $\mathcal{U}_{k}(\Phi)$ and $\mathcal{U}_{\aleph_{0}}(\Phi)$.

\subsection{Classifications of $\mathcal{E}$}

Our first result focuses on the classification of $\mathcal{E}$. For a set $X$ we denote by $|X|$ its cardinality.

Theorem 1. Let $\Phi=\left(E,\left\{f_{i}\right\}_{i=1}^{m}\right) \in \mathcal{E}$. Denote by $I=[a, b]$ the convex hull of $E$.

(A). The following statements are equivalent.

(i) $f_{1}(I) \cap f_{2}(I) \neq \emptyset$ or $f_{m-1}(I) \cap f_{m}(I) \neq \emptyset$.

(ii) $\operatorname{dim}_{H} \mathcal{U}_{k}(\Phi)=\operatorname{dim}_{H} \mathcal{U}_{1}(\Phi)$ for all integers $k \geq 1$.

(iii) $f_{1}(b) \in \mathcal{U}_{\aleph_{0}}(\Phi)$ or $f_{m}(a) \in \mathcal{U}_{\aleph_{0}}(\Phi)$.

(iv) $\left|\mathcal{U}_{\aleph_{0}}(\Phi)\right|=\aleph_{0}$.

(v) $\mathcal{U}_{1}(\Phi)$ is not closed.

(B). The following statements are also equivalent.

(i) $f_{1}(I) \cap f_{2}(I)=f_{m-1}(I) \cap f_{m}(I)=\emptyset$.

(ii) $\operatorname{dim}_{H} \mathcal{U}_{k}(\Phi)=\operatorname{dim}_{H} \mathcal{U}_{1}(\Phi)$ if $k=2^{\ell}$ with $\ell \in \mathbb{N} \cup\{0\}$, and $\mathcal{U}_{k}(\Phi)=\emptyset$ otherwise.

(iii) $f_{1}(b) \notin \mathcal{U}_{\aleph_{0}}(\Phi)$ and $f_{m}(a) \notin \mathcal{U}_{\aleph_{0}}(\Phi)$.

(iv) $\mathcal{U}_{\aleph_{0}}(\Phi)=\emptyset$.

(v) $\mathcal{U}_{1}(\Phi)$ is closed.

\section{Remark 1.1.}

- In the next result Theorem 2 we will show that for any integer $k \geq 1$ with $\mathcal{U}_{k}(\Phi) \neq \emptyset$ we must have $\operatorname{dim}_{H} \mathcal{U}_{k}(\Phi)>0$.

- Theorem 1 classifies the collection $\mathcal{E}$ and gives several dichotomies. For example, for any $\Phi \in \mathcal{E}$, either $\left|\mathcal{U}_{\aleph_{0}}(\Phi)\right|=\aleph_{0}$ or $\mathcal{U}_{\aleph_{0}}(\Phi)=\emptyset$.

- Although the closedness of $\mathcal{U}_{1}(\Phi)$ can be used to classify the elements of $\mathcal{E}$. This does not mean the same holds for $\mathcal{U}_{k}(\Phi)$ with $k \geq 2$. In fact, we show in Propositions 4.2 and 4.5 that for any $k \geq 2$ with $\mathcal{U}_{k}(\Phi) \neq \emptyset$, the set $\mathcal{U}_{k}(\Phi)$ is not closed.

\subsection{Hausdorff dimension and Hausdorff measure of $\mathcal{U}_{k}(E)$}

In general, without the complete overlap condition $(D)$ it is hard to calculate the Hausdorff dimensions of $E$ and $\mathcal{U}_{k}(\Phi)$. In our class $\mathcal{E}$ we are able to explicitly determine the Hausdorff dimensions of the attractor $E$ and the set $\mathcal{U}_{k}(\Phi)$. Furthermore, we show that for $k \geq 2$ with $\mathcal{U}_{k}(\Phi) \neq \emptyset$ the corresponding Hausdorff measure of $\mathcal{U}_{k}(\Phi)$ is always infinite. 
Definition 1.2. Given $\Phi=\left(E,\left\{f_{i}\right\}_{i=1}^{m}\right) \in \mathcal{E}$, the overlapping vectors $\mathbf{u}=\left(u_{1}, \ldots, u_{m}\right), \mathbf{v}=$ $\left(v_{1}, \ldots, v_{m}\right)$ of $\Phi$ are defined by

$$
\left\{\begin{array}{lll}
u_{i}=u, v_{i}=v & \text { if } & f_{i}(I) \cap f_{i+1}(I)=f_{i m^{u}}(I)=f_{(i+1) 1^{v}}(I) \\
u_{i}=v_{i}=\infty & \text { if } & f_{i}(I) \cap f_{i+1}(I)=\emptyset
\end{array}\right.
$$

Then by Definition $1.2 u_{m}$ and $v_{m}$ are always equal to $\infty$. Observe by Theorem 1 that $\mathcal{U}_{\aleph_{0}}(\Phi)$ is either a countable set or the empty set. So it suffices to consider the Hausdroff dimension and Hausdorff measure of $\mathcal{U}_{k}(\Phi)$ for $k=1,2, \ldots$ or $k=2^{\aleph_{0}}$.

Theorem 2. Let $\Phi=\left(E,\left\{f_{i}\right\}_{i=1}^{m}\right) \in \mathcal{E}$ with $f_{i}(x)=r_{i} x+b_{i}$ for $1 \leq i \leq m$, and let $\mathbf{u}=\left(u_{1}, \ldots, u_{m}\right), \mathbf{v}=\left(v_{1}, \ldots, v_{m}\right)$ be the overlapping vectors defined as in Definition 1.2.

(i) The Hausdorff dimensions of $\mathcal{U}_{2^{\aleph_{0}}}$ and $E$ are given by

$$
\operatorname{dim}_{H} \mathcal{U}_{2^{\aleph_{0}}}(\Phi)=\operatorname{dim}_{H} E=t
$$

where $t \in(0,1)$ is the root of

$$
\sum_{i=1}^{m} r_{i}^{t}\left(1-r_{m}^{u_{i} t}\right)=1
$$

Furthermore, the corresponding Hausdorff measures are positive and finite, i.e.,

$$
\mathcal{H}^{t}\left(\mathcal{U}_{2^{\aleph_{0}}}(\Phi)\right)=\mathcal{H}^{t}(E) \in(0, \infty)
$$

(ii) For any finite integer $k \geq 1$ satisfying $\mathcal{U}_{k}(\Phi) \neq \emptyset$, the Hausdorff dimension of $\mathcal{U}_{k}(\Phi)$ is given by

$$
\operatorname{dim}_{H} \mathcal{U}_{k}(\Phi)=\operatorname{dim}_{H} \mathcal{U}_{1}(\Phi)=s
$$

where $s \in(0,1)$ is the root of

$$
\sum_{i=1}^{m} r_{i}^{s}\left(1-\frac{r_{m}^{u_{i} s}\left(2-r_{1}^{v_{m-1} s}-r_{m}^{u_{1} s}\right)}{1-r_{1}^{v_{m-1} s} r_{m}^{u_{1} s}}\right)=1
$$

Furthermore, the corresponding Hausdorff measure of $\mathcal{U}_{k}(\Phi)$ admits

$$
\mathcal{H}^{s}\left(\mathcal{U}_{1}(\Phi)\right) \in(0, \infty)
$$

and

$$
\mathcal{H}^{s}\left(\mathcal{U}_{k}(\Phi)\right)=\infty \quad \text { for any } k \geq 2 \text { satisfying } \mathcal{U}_{k}(\Phi) \neq \emptyset
$$




\section{Remark 1.3.}

- By Propositions 5.3 and 5.5 the sets $\mathcal{U}_{1}(\Phi)$ and $E$ are both identical to strongly connected graph-directed sets satisfying the open set condition (OSC). So by [25] the results obtained in Theorem 2 also hold for packing dimension and corresponding packing measure.

- After the paper was finished we noticed that Theorem 2 (i) for the Hausdorff dimension of $E$ was also studied by Deng et al. [8, Theorem 8]. However, our method is different from theirs, where they determined the dimension of $E$ by considering an infinite iterated function system satisfying the OSC.

- The Hausdorff dimension formula for $\mathcal{U}_{k}(\Phi)$ described in Theorem 2 (ii) is in a compact form. If $\Phi=\left(E,\left\{f_{i}\right\}_{i=1}^{m}\right) \in \mathcal{E}$ with $f_{1}(I) \cap f_{2}(I)=f_{m-1}(I) \cap f_{m}(I)=\emptyset$, then $u_{1}=v_{m-1}=\infty$. By Theorem 2 (ii) the Hausdorff dimension of $\mathcal{U}_{k}(\Phi)$ can be simplified as $\operatorname{dim}_{H} \mathcal{U}_{k}(\Phi)=s$, where $s \in(0,1)$ is the root of

$$
1=\sum_{i=1}^{m} r_{i}^{s}\left(1-2 r_{m}^{u_{i} s}\right)
$$

Similarly, if $\Phi=\left(E,\left\{f_{i}\right\}_{i=1}^{m}\right) \in \mathcal{E}$ with $f_{1}(I) \cap f_{2}(I) \neq \emptyset$ and $f_{m-1}(I) \cap f_{m}(I)=\emptyset$, then $u_{1} \in \mathbb{N}$ and $v_{m-1}=\infty$. Again by Theorem 2 (ii) it follows that $\operatorname{dim}_{H} \mathcal{U}_{k}(\Phi)=s$ satisfies

$$
1=\sum_{i=1}^{m} r_{i}^{s}\left(1-2 r_{m}^{u_{i} s}+r_{m}^{\left(u_{i}+u_{1}\right) s}\right)
$$

Also, if $\Phi=\left(E,\left\{f_{i}\right\}_{i=1}^{m}\right) \in \mathcal{E}$ with $f_{1}(I) \cap f_{2}(I)=\emptyset$ and $f_{m-1}(I) \cap f_{m}(I) \neq \emptyset$, then $u_{1}=\infty$ and $v_{m-1} \in \mathbb{N}$. By Theorem 2 (ii) the Hausdorff dimension of $\mathcal{U}_{k}(\Phi)$ is given by $\operatorname{dim}_{H} \mathcal{U}_{k}(\Phi)=s$, where $s \in(0,1)$ is the root of

$$
1=\sum_{i=1}^{m} r_{i}^{s}\left(1-2 r_{m}^{u_{i} s}+r_{m}^{u_{i} s} r_{1}^{v_{m-1} s}\right)
$$

\subsection{Local dimension of a self-similar measure in $\mathcal{U}_{k}(\Phi)$ and $\mathcal{U}_{\aleph_{0}}(\Phi)$}

Given a probability vector $\mathbf{p}=\left(p_{1}, p_{2}, \ldots, p_{m}\right)$ with each $p_{i}>0$, let $\mu_{\mathbf{p}}$ be the selfsimilar measure defined on $\Phi=\left(E,\left\{f_{i}\right\}_{i=1}^{m}\right) \in \mathcal{E}$ (cf. [17]). Then

$$
\mu_{\mathbf{p}}=\sum_{i=1}^{m} p_{i} \mu_{\mathbf{p}} \circ f_{i}^{-1} .
$$

The measure $\mu_{\mathbf{p}}$ can also be deduced from the projection of Bernoulli measure on the symbolic space $\{1, \ldots, m\}^{\mathbb{N}}$. More precisely, let $\nu_{\mathbf{p}}$ be the Bernoulli measure on $\{1, \ldots, m\}^{\mathbb{N}}$ defined by 


$$
\nu_{\mathbf{p}}([i])=p_{i} \quad \text { for } \quad i=1, \ldots, m,
$$

where $[i]:=\left\{\left(j_{\ell}\right) \in\{1, \ldots, m\}^{\mathbb{N}}: j_{1}=i\right\}$ is a cylinder set. Then

$$
\mu_{\mathbf{p}}=\nu_{\mathbf{p}} \circ \pi^{-1}
$$

where $\pi$ is the projection map defined in (1.1).

Given $x \in E$, we define the lower and upper local dimensions of $\mu_{\mathbf{p}}$ at $x$ by

$$
\underline{\operatorname{dim}}_{l o c} \mu_{\mathbf{p}}(x):=\liminf _{r \rightarrow 0} \frac{\log \mu_{\mathbf{p}}(B(x, r))}{\log r}, \quad \overline{\operatorname{dim}}_{l o c} \mu_{\mathbf{p}}(x):=\limsup _{r \rightarrow 0} \frac{\log \mu_{\mathbf{p}}(B(x, r))}{\log r}
$$

where $B(x, r)=(x-r, x+r)$ is the open ball in $\mathbb{R}$ with center at $x$ and radius $r$. If the lower and upper local dimensions coincide, then the common value, denoted by $\operatorname{dim}_{l o c} \mu_{\mathbf{p}}(x)$, is called the local dimension of $\mu_{\mathbf{p}}$ at $x$.

To determine the local dimension of $\mu_{\mathbf{p}}$ is a central topic of multifractal analysis. Recently, Ngai and Xie [27] calculated the Hausdorff dimension of the self-similar measure $\mu_{\mathbf{p}}$, which provides a typical value of $\operatorname{dim}_{H} \mu_{\mathbf{p}}(x)$. For more results on the multifractal analysis of $\mu_{\mathbf{p}}$ we refer to the papers of Lau and Ngai [23], Feng and Lau [14], and references therein.

As a compensation of [27] we explicitly calculate the local dimension of $\mu_{\mathbf{p}}$ at points in $\mathcal{U}_{k}(\Phi)$ and $\mathcal{U}_{\aleph_{0}}(\Phi)$. For $n \in \mathbb{N}$ let $\{1, \ldots, m\}^{n}$ be the set of all length $n$ words over the alphabet $\{1, \ldots, m\}$. Denote by $\{1, \ldots, m\}^{*}=\bigcup_{n=0}^{\infty}\{1, \ldots, m\}^{n}$ the set of all finite words, where for $n=0$ we set $\{1, \ldots, m\}^{0}=\{\epsilon\}$ with $\epsilon$ the empty word.

Theorem 3. Let $\Phi=\left(E,\left\{f_{i}\right\}_{i=1}^{m}\right) \in \mathcal{E}$ with the convex hull conv $(E)=[a, b]$, and let $\mathbf{u}=\left(u_{1}, \ldots, u_{m}\right), \mathbf{v}=\left(v_{1}, \ldots, v_{m}\right)$ be the overlapping vector defined as in Definition 1.2.

(i) If $x \in \mathcal{U}_{k}(\Phi)$, then there exist a word $\mathbf{i} \in\{1, \ldots, m\}^{*}$ and a unique $y \in \mathcal{U}_{1}(\Phi)$ such that $x=f_{\mathbf{i}}(y)$. Furthermore,

$$
\begin{aligned}
& \underline{\operatorname{dim}}_{l o c} \mu_{\mathbf{p}}(x)=\underline{\operatorname{dim}}_{l o c} \mu_{\mathbf{p}}(y)=\liminf _{n \rightarrow \infty} \frac{\sum_{k=1}^{n} \log p_{j_{k}}}{\sum_{k=1}^{n} \log r_{j_{k}}}, \\
& \overline{\operatorname{dim}}_{l o c} \mu_{\mathbf{p}}(x)=\overline{\operatorname{dim}}_{l o c} \mu_{\mathbf{p}}(y)=\limsup _{n \rightarrow \infty} \frac{\sum_{k=1}^{n} \log p_{j_{k}}}{\sum_{k=1}^{n} \log r_{j_{k}}},
\end{aligned}
$$

where $j_{1} j_{2} \ldots$ is the unique coding of $y$.

(ii) If $x \in \mathcal{U}_{\aleph_{0}}(\Phi)$, then either $x=f_{\mathbf{i}}\left(f_{1}(b)\right)$ for some $\mathbf{i} \in\{1, \ldots, m\}^{*}$ and then

$$
\operatorname{dim}_{l o c} \mu_{\mathbf{p}}(x)=\min \left\{\frac{\log p_{m}}{\log r_{m}}, \frac{\log p_{2}+\left(v_{1}-1\right) \log p_{1}}{u_{1} \log r_{m}}\right\},
$$

or $x=f_{\mathbf{i}}\left(f_{m}(a)\right)$ for some $\mathbf{i} \in\{1, \ldots, m\}^{*}$ and thus 


$$
\operatorname{dim}_{l o c} \mu_{\mathbf{p}}(x)=\min \left\{\frac{\log p_{1}}{\log r_{1}}, \frac{\log p_{m-1}+\left(u_{m-1}-1\right) \log p_{m}}{v_{m-1} \log r_{1}}\right\}
$$

\subsection{An example}

At the end of this section we give an example to illustrate the main results Theorems $1-3$.

Example 1.4. Given two integers $M, N \geq 1$, let $\beta \in(1,4]$ satisfy

$$
\frac{4}{\beta}<1+\frac{1}{\beta^{M}}+\frac{1}{\beta^{N}}
$$

Define the maps

$$
\begin{array}{ll}
f_{1}(x)=\frac{x}{\beta}, & f_{2}(x)=\frac{x}{\beta}+\frac{1}{\beta}-\frac{1}{\beta^{M+1}}, \\
f_{3}(x)=\frac{x}{\beta}+1-\frac{2}{\beta}+\frac{1}{\beta^{N+1}}, & f_{4}(x)=\frac{x}{\beta}+1-\frac{1}{\beta} .
\end{array}
$$

Then there exists a unique non-empty compact set $E$ satisfying $E=\bigcup_{i=1}^{4} f_{i}(E)$. It is easy to check that the convex hull of $E$ is the unit interval $I=[0,1]$. The basic intervals $f_{i}(I)$ with $1 \leq i \leq 4$ are plotted as in Fig. 1. The inequality in (1.2) guarantees that $f_{2}(I) \cap f_{3}(I)=\emptyset$. Furthermore, one can verify that

$$
\begin{aligned}
& f_{1}(I) \cap f_{2}(I)=f_{14^{M}}(I)=f_{21^{M}}(I), \\
& f_{3}(I) \cap f_{4}(I)=f_{34^{N}}(I)=f_{41^{N}}(I) .
\end{aligned}
$$

So, $\Phi=\left(E,\left\{f_{i}\right\}_{i=1}^{4}\right) \in \mathcal{E}$ for any integers $M, N \geq 1$.

First, by Theorems 1 and 2 it follows that for any $k \in \mathbb{N}$,

$$
\operatorname{dim}_{H} \mathcal{U}_{k}(\Phi)=\operatorname{dim}_{H} \mathcal{U}_{1}(\Phi)=s
$$

where $s \in(0,1)$ satisfies

$$
\beta^{s}+2 \beta^{N s}+2 \beta^{M s}=4+\beta^{(N-M) s}+\beta^{(M-N) s} .
$$

Moreover, the corresponding Hausdorff measures satisfy

$$
\mathcal{H}^{s}\left(\mathcal{U}_{1}(\Phi)\right) \in(0, \infty), \quad \text { and } \quad \mathcal{H}^{s}\left(\mathcal{U}_{k}(\Phi)\right)=\infty \quad \forall k \geq 2
$$

Second, by Proposition 3.4 it follows that $\mathcal{U}_{\aleph_{0}}(\Phi)$ is a countable set consisting of all points with a coding ending with either $14^{\infty}$ or $41^{\infty}$. Furthermore, by Theorem 2 it follows that the Hausdorff dimensions of $\mathcal{U}_{2^{\aleph_{0}}}(\Phi)$ and $E$ are given by 


$$
\operatorname{dim}_{H} \mathcal{U}_{2^{\aleph_{0}}}(\Phi)=\operatorname{dim}_{H} E=t
$$

where $t \in(0,1)$ satisfies

$$
\beta^{t}+\beta^{-M t}+\beta^{-N t}=4
$$

And the corresponding Hausdorff measures admit

$$
\mathcal{H}^{t}\left(\mathcal{U}_{2^{\aleph_{0}}}(\Phi)\right)=\mathcal{H}^{t}(E) \in(0, \infty)
$$

Third, for a given probability vector $\mathbf{p}=\left(p_{1}, p_{2}, p_{3}, p_{4}\right)$ with each $p_{i}>0$ we define the corresponding self-similar measure $\mu_{\mathbf{p}}$ by

$$
\mu_{\mathbf{p}}=\sum_{i=1}^{4} p_{i} \mu_{\mathbf{p}} \circ f_{i}^{-1}
$$

Then by Theorem 3 it follows that for any $x \in \mathcal{U}_{k}(\Phi)$ there exists a unique $y \in \mathcal{U}_{1}(\Phi)$ such that

$$
\begin{aligned}
& \underline{\operatorname{dim}}_{l o c} \mu_{\mathbf{p}}(x)=\underline{\operatorname{dim}}_{l o c} \mu_{\mathbf{p}}(y)=\frac{-1}{\log \beta} \limsup _{n \rightarrow \infty} \frac{\sum_{k=1}^{n} \log p_{j_{k}}}{n}, \\
& \overline{\operatorname{dim}}_{l o c} \mu_{\mathbf{p}}(x)=\overline{\operatorname{dim}}_{l o c} \mu_{\mathbf{p}}(y)=\frac{-1}{\log \beta} \liminf _{n \rightarrow \infty} \frac{\sum_{k=1}^{n} \log p_{j_{k}}}{n},
\end{aligned}
$$

where $j_{1} j_{2} \ldots$ is the unique coding of $y$. Moreover, for any $x \in \mathcal{U}_{\aleph_{0}}(\Phi)$ we have the following two cases:

$$
x=f_{\mathbf{i}}\left(\frac{1}{\beta}\right) \quad \text { or } \quad x=f_{\mathbf{i}}\left(1-\frac{1}{\beta}\right)
$$

for some $\mathbf{i} \in\{1,2,3,4\}^{*}$. If $x=f_{\mathbf{i}}\left(\frac{1}{\beta}\right)$, then

$$
\operatorname{dim}_{l o c} \mu_{\mathbf{p}}(x)=\min \left\{\frac{\log p_{4}}{-\log \beta}, \frac{\log p_{2}+(M-1) \log p_{1}}{-M \log \beta}\right\} .
$$

If $x=f_{\mathbf{i}}\left(1-\frac{1}{\beta}\right)$, then

$$
\operatorname{dim}_{l o c} \mu_{\mathbf{p}}(x)=\min \left\{\frac{\log p_{1}}{-\log \beta}, \frac{\log p_{3}+(N-1) \log p_{4}}{-N \log \beta}\right\} .
$$

In particular, if $p_{i}=\frac{1}{4}$ for all $1 \leq i \leq 4$, then

$$
\operatorname{dim}_{l o c} \mu_{\mathbf{p}}(x)=\frac{\log 4}{\log \beta} \quad \text { for all } x \in \mathcal{U}_{\aleph_{0}}(\Phi) .
$$


The rest of the paper is organized as follows. The proof of Theorem 1 is given in Sections 2-4. First in Section 2 we characterize the set of points in $E$ with finitely many codings, and prove the equivalence (i) $\Leftrightarrow$ (ii) in Theorem 1 (A) and (B). Second in Section 3 we describe the set of points in $E$ with countably many codings, and establish the equivalence (i) $\Leftrightarrow$ (iii) $\Leftrightarrow$ (iv). Finally in Section 4 we investigate the topology of $\mathcal{U}_{k}(\Phi)$, and deduce the equivalence $(\mathrm{i}) \Leftrightarrow(\mathrm{v})$. In Section 5 we show that the univoque set $\mathcal{U}_{1}(\Phi)$ and the self-similar set $E$ are both identical to the strongly connected graphdirected sets satisfying the OSC. Based on this we prove Theorem 2 in Section 6 for the Hausdorff dimensions and Hausdorff measures of $E$ and $\mathcal{U}_{k}(\Phi)$. In particular, we show that the corresponding Hausdorff measure of $\mathcal{U}_{k}(\Phi)$ is always infinite for any $k \geq 2$ satisfying $\mathcal{U}_{k}(\Phi) \neq \emptyset$. In Section 7 we prove Theorem 3 for the local dimension of the self-similar measure $\mu_{\mathbf{p}}$ at points in $\mathcal{U}_{k}(\Phi)$ and $\mathcal{U}_{\aleph_{0}}(\Phi)$. Finally, in Section 8 we pose some remarks on a possible extension of our class $\mathcal{E}$.

\section{Finitely many codings}

Let $\Phi=\left(E,\left\{f_{i}\right\}_{i=1}^{m}\right) \in \mathcal{E}$ and $k \in \mathbb{N}$. In this section we will consider the set $\mathcal{U}_{k}(\Phi)$ consisting of all $x \in E$ having precisely $k$ different codings with respect to $\left\{f_{i}\right\}_{i=1}^{m}$, and prove the equivalence $(i) \Leftrightarrow(i i)$ in Theorems 1 (A) and (B), respectively.

Let $\{1, \ldots, m\}^{\mathbb{N}}$ be the set of sequences $\left(d_{i}\right)$ with each $d_{i} \in\{1, \ldots, m\}$, and let $\{1, \ldots, m\}^{*}$ be the set of all finite words over the alphabet $\{1, \ldots, m\}$. For any two words $\mathbf{c}=c_{1} \ldots c_{m}, \mathbf{d}=d_{1} \ldots d_{n} \in\{1, \ldots, m\}^{*}$ we let $\mathbf{c d}=c_{1} \ldots c_{m} d_{1} \ldots d_{n}$ denote their concatenation. Moreover, for $k \in \mathbb{N}$ we write $\mathbf{c}^{k}=\mathbf{c c} \cdots \mathbf{c}$ the $k$ times concatenation of $\mathbf{c}$ with itself, and let $\mathbf{c}^{\infty}$ denote the infinite concatenation of $\mathbf{c}$ with itself. Recall that $\left\{f_{i}\right\}_{i=1}^{m}$ is the collection of contractive similitudes. For a word $\mathbf{d}=d_{1} d_{2} \ldots d_{n} \in\{1, \ldots, m\}^{*}$ we let $f_{\mathbf{d}}=f_{d_{1}} \circ f_{d_{2}} \circ \cdots \circ f_{d_{n}}$ denote the compositions of the maps $f_{d_{1}}, \ldots, f_{d_{n}}$. In particular, for the empty word $\epsilon$ we set $f_{\epsilon}$ the identity map.

For a set $F \subset \mathbb{R}$ we denote by $\operatorname{conv}(F)$ the convex hull of $F$.

Lemma 2.1. Let $\left(E,\left\{f_{i}\right\}_{i=1}^{m}\right) \in \mathcal{E}$ with $I=\operatorname{conv}(E)$. If $f_{i^{u}}(I)=f_{(i+1) 1^{v}}(I)$ for some $i \in\{1, \ldots, m-1\}$ and $u, v \in \mathbb{N}$, then $f_{i m^{u}}(\cdot)=f_{(i+1) 1^{v}}(\cdot)$.

Proof. Note that for any $x \in \mathbb{R}$ we can write

$$
f_{i m^{u}}(x)=r x+t, \quad f_{(i+1) 1^{v}}(x)=r^{\prime} x+t^{\prime},
$$

for some $r, r^{\prime} \in(0,1)$ and $t, t^{\prime} \in \mathbb{R}$. Suppose that $I=[a, b]$. Then by using $f_{i m^{u}}(I)=$ $f_{(i+1) 1^{v}}(I)$ it follows that

$$
\begin{aligned}
& r a+t=f_{i m^{u}}(a)=f_{(i+1) 1^{v}}(a)=r^{\prime} a+t^{\prime}, \\
& r b+t=f_{i m^{u}}(b)=f_{(i+1) 1^{v}}(b)=r^{\prime} b+t^{\prime} .
\end{aligned}
$$

This implies $r=r^{\prime}$ and $t=t^{\prime}$. By $(2.1)$ we have $f_{i m^{u}}(\cdot)=f_{(i+1) 1^{v}}(\cdot)$. 
Lemma 2.2. Let $\left(E,\left\{f_{i}\right\}_{i=1}^{m}\right) \in \mathcal{E}$ with $I=\operatorname{conv}(E)$. If

$$
f_{i}(I) \cap f_{i+1}(I)=f_{i m^{u}}(I)=f_{(i+1) 1^{v}}(I)
$$

for some $i \in\{1, \cdots, m-1\}$ and $u, v \in \mathbb{N}$, then for any $x \in f_{i}(I) \cap f_{i+1}(I) \cap E$ all of its codings either begin with $i^{u-1}$ or begin with $(i+1) 1^{v-1}$.

Proof. Let $\left(d_{i}\right)$ be a coding of $x$ with respect to $\left\{f_{i}\right\}_{i=1}^{m}$. Note that $\pi\left(d_{1} d_{2} \ldots\right)=x \in$ $f_{i}(I) \cap f_{i+1}(I) \cap E$ and that any three basic intervals have an empty intersection. Then

$$
d_{1}=i \quad \text { or } \quad d_{1}=i+1
$$

If $d_{1}=i$ with $u=1$ or $d_{1}=i+1$ with $v=1$, then we are done. So, we will finish the proof by considering the following two cases.

Case (I). $d_{1}=i$ and $u>1$. Note that $x=\pi\left(i d_{2} d_{3} \cdots\right) \in f_{i m^{u}}(I)$. Then

$$
\pi\left(d_{2} d_{3} \cdots\right) \in f_{m^{u}}(I)
$$

and we claim that $d_{2}=m$.

Suppose on the contrary that $d_{2} \neq m$. Then in view of the location of these basic intervals we have $d_{2}=m-1$. So, by (2.2) and Condition (D) it follows that

$$
\pi\left(d_{2} d_{3} \cdots\right) \in f_{m^{u}}(I) \cap\left(f_{m-1}(I) \cap f_{m}(I)\right) \subseteq f_{m^{u}}(I) \cap f_{m 1}(I),
$$

leading to a contradiction with $f_{1}(I) \cap f_{m}(I)=\emptyset$. Therefore, $d_{2}=m$. Iterating the above procedure it follows that $d_{2} \cdots d_{u}=m^{u-1}$.

Case (II). $d_{1}=i+1$ and $v>1$. Note that $x=\pi\left((i+1) d_{2} d_{3} \cdots\right) \in f_{(i+1) 1^{v}}(I)$. Then

$$
\pi\left(d_{2} d_{3} \cdots\right) \in f_{1^{v}}(I)
$$

and we will prove that $d_{2}=1$. If $d_{2} \neq 1$, then $d_{2}=2$. So, by (2.3) and Condition (D) it follows that

$$
\pi\left(d_{2} d_{3} \cdots\right) \in f_{1^{v}}(I) \cap\left(f_{1}(I) \cap f_{2}(I)\right) \subseteq f_{1^{v}}(I) \cap f_{1 m}(I)
$$

leading to a contradiction with $f_{1}(I) \cap f_{m}(I)=\emptyset$. Hence $d_{2}=1$. By iteration we conclude that $d_{2} \cdots d_{v}=1^{v-1}$.

Now we turn to show that the Hausdorff dimensions of $\mathcal{U}_{k}(\Phi)$ and $\mathcal{U}_{1}(\Phi)$ coincide for any $k \geq 2$ satisfying $\mathcal{U}_{k}(\Phi) \neq \emptyset$. Later on in Section 6 we will explicitly calculate the Hausdorff dimension of $\mathcal{U}_{1}(\Phi)$. In particular, we have $\operatorname{dim}_{H} \mathcal{U}_{1}(\Phi)>0$ for any $\Phi \in \mathcal{E}$. The upper bound of $\operatorname{dim}_{H} \mathcal{U}_{k}(\Phi)$ follows directly. 
Lemma 2.3. Let $\Phi=\left(E,\left\{f_{i}\right\}_{i=1}^{m}\right) \in \mathcal{E}$. Then for any $k \in \mathbb{N}$ we have

$$
\operatorname{dim}_{H} \mathcal{U}_{k}(\Phi) \leq \operatorname{dim}_{H} \mathcal{U}_{1}(\Phi)
$$

Proof. Take $x \in \mathcal{U}_{k}(\Phi)$. Then all codings of $x$ will eventually end in

$$
\pi^{-1}\left(\mathcal{U}_{1}(\Phi)\right)=\left\{\left(c_{i}\right) \in\{1, \cdots, m\}^{\infty}: \pi\left(\left(c_{i}\right)\right) \in \mathcal{U}_{1}(\Phi)\right\}
$$

In other words,

$$
\mathcal{U}_{k}(\Phi) \subseteq \underset{d_{1} \cdots d_{n} \in\{1,2, \cdots, m\}^{*}}{\bigcup_{d_{1} \cdots d_{n}}\left(\mathcal{U}_{1}(\Phi)\right)}
$$

Therefore, the lemma follows by the countable stability of Hausdorff dimension (cf. [13]).

For the lower bound of $\operatorname{dim}_{H} \mathcal{U}_{k}(E)$ we split the proof into the following two subsections.

(i) $f_{1}(I) \cap f_{2}(I) \neq \emptyset$ or $f_{m-1}(I) \cap f_{m}(I) \neq \emptyset ; \quad(i i) f_{1}(I) \cap f_{2}(I)=f_{m-1}(I) \cap f_{m}(I)=\emptyset$.

\section{1. $f_{1}(I) \cap f_{2}(I) \neq \emptyset$ or $f_{m-1}(I) \cap f_{m}(I) \neq \emptyset$}

Let $\Phi=\left(E,\left\{f_{i}\right\}_{i=1}^{m}\right) \in \mathcal{E}$ with convex hull $I=\operatorname{conv}(E)$. By Condition $(\mathrm{C})$ there exists $i_{0} \in\{1, \ldots, m-1\}$ such that $f_{i_{0}}(I) \cap f_{i_{0}+1}(I)=\emptyset$. In the following lemma we show that the Hausdorff dimension of $\mathcal{U}_{1}(\Phi)$ is dominated by that of the subset consisting of all $x \in \mathcal{U}_{1}(E)$ whose unique coding starts at digit $i_{0}$ or $i_{0}+1$.

Lemma 2.4. Let $\Phi=\left(E,\left\{f_{i}\right\}_{i=1}^{m}\right) \in \mathcal{E}$ with $I=\operatorname{conv}(E)$. If $f_{i_{0}}(I) \cap f_{i_{0}+1}(I)=\emptyset$ for some $i_{0} \in\{1, \cdots, m-1\}$, then

$$
\operatorname{dim}_{H} \mathcal{U}_{1}(\Phi)=\operatorname{dim}_{H}\left(f_{i_{0}}(E) \cap \mathcal{U}_{1}(\Phi)\right)=\operatorname{dim}_{H}\left(f_{i_{0}+1}(E) \cap \mathcal{U}_{1}(\Phi)\right)
$$

Proof. Note that $\mathcal{U}_{1}(\Phi)=\bigcup_{j=1}^{m}\left(f_{j}(E) \cap \mathcal{U}_{1}(\Phi)\right)$. It suffices to prove

$$
\operatorname{dim}_{H}\left(f_{i_{0}}(E) \cap \mathcal{U}_{1}(\Phi)\right) \geq \operatorname{dim}_{H}\left(\bigcup_{j=i_{0}+1}^{m} f_{j}(E) \cap \mathcal{U}_{1}(\Phi)\right)
$$

and

$$
\operatorname{dim}_{H}\left(f_{i_{0}+1}(E) \cap \mathcal{U}_{1}(\Phi)\right) \geq \operatorname{dim}_{H}\left(\bigcup_{j=1}^{i_{0}} f_{j}(E) \cap \mathcal{U}_{1}(\Phi)\right)
$$


Without loss of generality we only prove (2.4). Let

$$
\begin{aligned}
\varphi: \bigcup_{j=i_{0}+1}^{m} f_{j}(E) \cap \mathcal{U}_{1}(\Phi) & \longrightarrow f_{i_{0}}(E) \cap \mathcal{U}_{1}(\Phi) \\
x & \mapsto f_{i_{0}}(x) .
\end{aligned}
$$

First we prove that $\varphi$ is well-defined. Take $x \in \bigcup_{j=i_{0}+1}^{m} f_{j}(E) \cap \mathcal{U}_{1}(\Phi)$. It suffices to prove that $f_{i_{0}}(x) \in \mathcal{U}_{1}(\Phi)$. Suppose on the contrary that $f_{i_{0}}(x) \notin \mathcal{U}_{1}(\Phi)$. Note that $f_{i_{0}}(I) \cap f_{i_{0}+1}(I)=\emptyset$. Then by the locations of the fundamental intervals it follows that

$$
f_{i_{0}}(x) \in f_{i_{0}-1}(I) \cap f_{i_{0}}(I) \subseteq f_{i_{0} 1}(I) .
$$

This implies that $x \in f_{1}(I)$, leading to a contradiction with $f_{1}(I) \cap \bigcup_{j=i_{0}+1}^{m} f_{j}(I)=\emptyset$.

Therefore, $\varphi$ is well-defined. Note that $\varphi$ is a similitude. Hence, by [13, Proposition 3.3] it follows that

$$
\begin{aligned}
\operatorname{dim}_{H}\left(f_{i_{0}}(E) \cap \mathcal{U}_{1}(\Phi)\right) & \geq \operatorname{dim}_{H} \varphi\left(\bigcup_{j=i_{0}+1}^{m} f_{j}(E) \cap \mathcal{U}_{1}(\Phi)\right) \\
& =\operatorname{dim}_{H}\left(\bigcup_{j=i_{0}+1}^{m} f_{j}(E) \cap \mathcal{U}_{1}(\Phi)\right)
\end{aligned}
$$

This establishes (2.4).

Lemma 2.5. Let $\Phi=\left(E,\left\{f_{i}\right\}_{i=1}^{m}\right) \in \mathcal{E}$ with $I=\operatorname{conv}(E)$, and let $k \in \mathbb{N}$.

(i) If $f_{1}(I) \cap f_{2}(I)=f_{1 m^{u}}(I)=f_{21^{v}}(I)$ and $f_{i_{0}}(I) \cap f_{i_{0}+1}(I)=\emptyset$ for some $i_{0} \in$ $\{2, \ldots, m-1\}$, then

$$
\pi\left(1 m^{u(k-1)} \mathbf{c}\right) \in \mathcal{U}_{k}(\Phi)
$$

for any $\pi(\mathbf{c}) \in f_{i_{0}}(E) \cap \mathcal{U}_{1}(\Phi)$.

(ii) If $f_{m-1}(I) \cap f_{m}(I)=f_{(m-1) m^{u}}(I)=f_{m 1^{v}}(I)$ and $f_{i_{0}}(I) \cap f_{i_{0}+1}(I)=\emptyset$ for some $i_{0} \in\{1, \ldots, m-2\}$, then

$$
\pi\left(m 1^{v(k-1)} \mathbf{c}\right) \in \mathcal{U}_{k}(\Phi)
$$

for any $\pi(\mathbf{c}) \in f_{i_{0}+1}(E) \cap \mathcal{U}_{1}(\Phi)$.

Proof. Since the proof of (ii) is similar, we only prove (i).

Take $\pi(\boldsymbol{c})=\pi\left(\left(c_{i}\right)\right) \in f_{i_{0}}(E) \cap \mathcal{U}_{1}(\Phi)$. Then $c_{1}=i_{0}$. It suffices to prove that for any integer $k \geq 0$, 


$$
z_{k}:=\pi\left(1 m^{u k} \boldsymbol{c}\right)
$$

has exactly $k+1$ different codings. We will prove this by induction on $k$. Suppose $k=0$. Then $z_{0}=\pi(1 \boldsymbol{c})$. Note that $f_{i_{0}}(I) \cap f_{i_{0}+1}(I)=\emptyset$ for some $i_{0} \in\{2, \cdots, m-1\}$. Denote by $I=[a, b]$. Then by Condition (A) it follows that

$$
z_{0}=\pi\left(1 i_{0} c_{2} c_{3} \cdots\right) \leq f_{1 i_{0}}(b)<f_{1 m^{u}}(a)=f_{21^{v}}(a)=f_{2}(a)
$$

where the equality $f_{1 m^{u}}(a)=f_{21^{v}}(a)$ follows by Lemma 2.1. This together with $\pi(\boldsymbol{c})=$ $\pi\left(i_{0} c_{2} c_{3} \cdots\right) \in \mathcal{U}_{1}(\Phi)$ implies that $z_{0} \in \mathcal{U}_{1}(\Phi)$.

Now suppose that $z_{k}$ has $k+1$ different codings for some $k \geq 0$. We will prove that $z_{k+1}$ has exactly $k+2$ different codings. Note that

$$
z_{k+1}=f_{1 m^{u}}\left(\pi\left(m^{u k} \boldsymbol{c}\right)\right)=f_{21^{v}}\left(\pi\left(m^{u k} \boldsymbol{c}\right)\right)=f_{21^{v-1}}\left(z_{k}\right)
$$

By the induction hypothesis this implies that $z_{k+1}$ has at least $k+2$ different codings: one is $1 m^{u(k+1)} \boldsymbol{c}$, and the others start at $21^{v-1}$. In the following we show that $z_{k+1}$ has precisely $k+2$ different codings. Suppose $\left(d_{i}\right)$ is a coding of $z_{k+1}$. Then by $(2.5)$ and Lemma 2.2 it follows that

$$
d_{1} \cdots d_{u}=1 m^{u-1} \quad \text { or } \quad d_{1} \cdots d_{v}=21^{v-1}
$$

By the induction hypothesis it suffices to prove that $d_{1} \cdots d_{u}=1 m^{u-1}$ implies $\left(d_{i}\right)=$ $1 m^{u(k+1)}$ c.

Suppose $d_{1} \cdots d_{u}=1 m^{u-1}$. We claim that $d_{u+1} \cdots d_{u(k+1)+1}=m^{u k+1}$. Let $j \in$ $\{1, \ldots, u k+1\}$ be the smallest integer such that $d_{u+j} \neq m$. Then by (2.5) we have

$$
\pi\left(d_{u+j} d_{u+j+1} \cdots\right)=f_{m^{u k+2-j}}(\pi(\boldsymbol{c}))
$$

In view of the locations of the basic intervals we have $d_{u+j}=m-1$. Therefore, by (2.6) and Condition (D) it follows that

$$
f_{m^{u k+2-j}}(\pi(\boldsymbol{c})) \in f_{m-1}(I) \cap f_{m}(I) \subseteq f_{m 1}(I) .
$$

This implies that $f_{m^{u k+1-j}}(\pi(\boldsymbol{c})) \in f_{1}(I)$. If $j<u k+1$, then we obtain $f_{m}(I) \cap f_{1}(I) \neq \emptyset$, leading to a contradiction with $f_{i_{0}}(I) \cap f_{i_{0}+1}(I)=\emptyset$. If $j=u k+1$, then we get $\pi(\boldsymbol{c}) \in$ $f_{1}(I)$, leading to a contradiction with $\pi(\boldsymbol{c})=\pi\left(i_{0} c_{2} c_{3} \cdots\right) \in \mathcal{U}_{1}(\Phi)$ and $i_{0} \geq 2$. Thus, $d_{u+1} \ldots d_{u(k+1)+1}=m^{u k+1}$. Since $\pi(\mathbf{c}) \in \mathcal{U}_{1}(\Phi)$, we conclude that $\left(d_{i}\right)=1 m^{u(k+1)} \boldsymbol{c}$.

Hence, by induction it follows that $z_{k}$ has exactly $k+1$ different codings for any integer $k \geq 0$. This establishes (i).

Lemma 2.6. Let $\Phi=\left(E,\left\{f_{i}\right\}_{i=1}^{m}\right) \in \mathcal{E}$ with $I=\operatorname{conv}(E)$. If $f_{1}(I) \cap f_{2}(I) \neq \emptyset$ or $f_{m-1}(I) \cap$ $f_{m}(I) \neq \emptyset$, then 


$$
\operatorname{dim}_{H} \mathcal{U}_{k}(E) \geq \operatorname{dim}_{H} \mathcal{U}_{1}(E) \quad \forall k \in \mathbb{N}
$$

Proof. Without loss of generality we assume $f_{1}(I) \cap f_{2}(I) \neq \emptyset$. By Condition (C) there exists $i_{0} \in\{2, \ldots, m-1\}$ such that $f_{i_{0}}(I) \cap f_{i_{0}+1}(I)=\emptyset$. Then by Lemma 2.4 it follows that

$$
\operatorname{dim}_{H} \mathcal{U}_{1}(\Phi)=\operatorname{dim}_{H}\left(f_{i_{0}}(E) \cap \mathcal{U}_{1}(\Phi)\right)
$$

Note that $f_{1}(I) \cap f_{2}(I) \neq \emptyset$. Then by Condition (D) there exist $u=u_{1}, v=v_{1} \in \mathbb{N}$ such that $f_{1}(I) \cap f_{2}(I)=f_{1 m^{u}}(I)=f_{21^{v}}(I)$. So by Lemma 2.5 (i) it follows that

$$
\left\{\pi\left(1 m^{u(k-1)} \boldsymbol{c}\right): \pi(\boldsymbol{c}) \in f_{i_{0}}(E) \cap \mathcal{U}_{1}(\Phi)\right\} \subseteq \mathcal{U}_{k}(\Phi)
$$

Hence, by (2.7) we conclude that

$$
\operatorname{dim}_{H} \mathcal{U}_{k}(\Phi) \geq \operatorname{dim}_{H}\left(f_{i_{0}}(E) \cap \mathcal{U}_{1}(\Phi)\right)=\operatorname{dim}_{H} \mathcal{U}_{1}(\Phi)
$$

2.2. $f_{1}(I) \cap f_{2}(I)=f_{m-1}(I) \cap f_{m}(I)=\emptyset$

Let $\Phi=\left(E,\left\{f_{i}\right\}_{i=1}^{m}\right) \in \mathcal{E}$. Different from the previous subsection it happens in this case that $\mathcal{U}_{k}(\Phi)=\emptyset$ for some $k \in \mathbb{N}$.

Lemma 2.7. Let $\Phi=\left(E,\left\{f_{i}\right\}_{i=1}^{m}\right) \in \mathcal{E}$ with $I=\operatorname{conv}(E)$. If $f_{1}(I) \cap f_{2}(I)=f_{m-1}(I) \cap$ $f_{m}(I)=\emptyset$, then

$$
\mathcal{U}_{k}(\Phi)=\emptyset \quad \text { for any } k \neq 2^{\ell} \text { with } \ell \in \mathbb{N} \cup\{0\}
$$

Proof. For $x \in E$ we denote by $N(x)$ the number of different codings of $x$ with respect to $\left\{f_{i}\right\}_{i=1}^{m}$. Let $k \geq 2$ and take $x \in \mathcal{U}_{k}(\Phi)$. Then $N(x)=k$. So, there exists

$$
x_{1} \in \mathcal{U}_{k}(\Phi) \cap f_{i}(I) \cap f_{i+1}(I)
$$

for some $i \in\{2, \ldots, m-2\}$ such that $N(x)=N\left(x_{1}\right)$. Note that $f_{i}(I) \cap f_{i+1}(I) \neq \emptyset$. Then by Condition (D) there exist $u=u_{i}, v=v_{i} \in \mathbb{N}$ such that

$$
f_{i}(I) \cap f_{i+1}(I)=f_{i m^{u}}(I)=f_{(i+1) 1^{v}}(I)
$$

Observe that $f_{1}(I) \cap f_{2}(I)=f_{m-1}(I) \cap f_{m}(I)=\emptyset$. Then in view of the locations of these basic intervals we obtain

$$
f_{1}(I) \cap f_{i}(I)=\emptyset \quad \forall i \neq 1 \quad \text { and } \quad f_{j}(I) \cap f_{m}(I)=\emptyset \quad \forall j \neq m
$$

Therefore, by (2.8)-(2.10) and an argument similar to the one in the proof of Lemma 2.2 it follows that all codings of $x_{1}$ either begin with $i m^{u}$ or begin with $(i+1) 1^{v}$. 
By (2.9) and Lemma 2.1 it gives that $f_{i m^{u}}(\cdot)=f_{(i+1) 1^{v}}(\cdot)$. Then there exists a unique $y \in E$ such that $x_{1}=f_{i m^{u}}(y)=f_{(i+1) 1^{v}}(y)$. Furthermore,

$$
N\left(x_{1}\right)=N\left(f_{m^{u}}(y)\right)+N\left(f_{1^{v}}(y)\right)=2 N(y),
$$

where the last equality holds by (2.10) that

$$
N\left(f_{m^{u}}(y)\right)=N(y)=N\left(f_{1^{v}}(y)\right) .
$$

Hence, we conclude that $N(x)=N\left(x_{1}\right)=2 N(y)$.

By iteration of the above arguments it follows that $N(x)$ must be of the form $2^{\ell}$ for some $\ell \in \mathbb{N} \cup\{0\}$. This completes the proof.

In fact the condition $k \neq 2^{\ell}$ in the above lemma is also a necessary condition for $\mathcal{U}_{k}(\Phi)=\emptyset$.

Lemma 2.8. Let $\Phi=\left(E,\left\{f_{i}\right\}_{i=1}^{m}\right) \in \mathcal{E}$ with $I=\operatorname{conv}(E)$. If $f_{1}(I) \cap f_{2}(I)=f_{m-1}(I) \cap$ $f_{m}(I)=\emptyset$, then

$$
\operatorname{dim}_{H} \mathcal{U}_{2^{\ell}}(\Phi) \geq \operatorname{dim}_{H} \mathcal{U}_{1}(\Phi) \quad \forall \ell \in \mathbb{N} \cup\{0\}
$$

Proof. We will prove the inequality $\operatorname{dim}_{H} \mathcal{U}_{2}(\Phi) \geq \operatorname{dim}_{H} \mathcal{U}_{1}(\Phi)$ by induction on $\ell$. Trivially this inequality holds if $\ell=0$. Now we assume this inequality holds for some $\ell \geq 0$. It suffices to prove

$$
\operatorname{dim}_{H} \mathcal{U}_{2^{\ell+1}}(\Phi) \geq \operatorname{dim}_{H} \mathcal{U}_{2^{\ell}}(\Phi)
$$

By Condition (C) there exists $i \in\{2, \cdots, m-2\}$ such that $f_{i}(I) \cap f_{i+1}(I) \neq \emptyset$. Then Condition (D) gives two indexes $u=u_{i}, v=v_{i} \in \mathbb{N}$ such that

$$
f_{i}(I) \cap f_{i+1}(I)=f_{i m^{u}}(I)=f_{(i+1) 1^{v}}(I) .
$$

By Lemma 2.1 this yields that $f_{i m^{u}}(\cdot)=f_{(i+1) 1^{v}}(\cdot)$. Note that any three basic intervals have an empty intersection. So, by (2.10) it follows that

$$
\left\{f_{i m^{u}}(x)=f_{(i+1) 1^{v}}(x): x \in \mathcal{U}_{2^{\ell}}(\Phi)\right\} \subseteq \mathcal{U}_{2^{\ell+1}}(\Phi)
$$

which implies $\operatorname{dim}_{H} \mathcal{U}_{2^{\ell+1}}(\Phi) \geq \operatorname{dim}_{H} \mathcal{U}_{2^{\ell}}(\Phi)$.

By induction we conclude that $\operatorname{dim}_{H} \mathcal{U}_{2}(\Phi) \geq \operatorname{dim}_{H} \mathcal{U}_{1}(\Phi)$ for any $\ell \in \mathbb{N}$.

Proof of $(i) \Leftrightarrow(i i)$ in Theorems 1 (A) and (B). Let $\Phi=\left(E,\left\{f_{i}\right\}_{i=1}^{m}\right) \in \mathcal{E}$ with $I=$ $\operatorname{conv}(E)$. By Lemmas 2.3 and 2.6 it follows that if $f_{1}(I) \cap f_{2}(I) \neq \emptyset$ or $f_{m-1}(I) \cap f_{m}(I) \neq$ $\emptyset$, then 


$$
\operatorname{dim}_{H} \mathcal{U}_{k}(\Phi)=\operatorname{dim}_{H} \mathcal{U}_{1}(\Phi) \quad \text { for all } \quad k \in \mathbb{N}
$$

On the other hand, if $f_{1}(I) \cap f_{2}(I)=f_{m-1}(I) \cap f_{m}(I)=\emptyset$, then by Lemmas 2.3, 2.7 and 2.8 it follows that

$$
\begin{cases}\operatorname{dim}_{H} \mathcal{U}_{k}(\Phi)=\operatorname{dim}_{H} \mathcal{U}_{1}(\Phi) & \text { if } k=2^{\ell} \text { with } \ell \in \mathbb{N} \cup\{0\} \\ \mathcal{U}_{k}(\Phi)=\emptyset & \text { otherwise }\end{cases}
$$

Therefore, the equivalence (i) $\Leftrightarrow$ (ii) holds true.

\section{Countably many codings}

Given $\Phi=\left(E,\left\{f_{i}\right\}_{i=1}^{m}\right) \in \mathcal{E}$, we will consider in this section the set $\mathcal{U}_{\aleph_{0}}(\Phi)$ of points having countably many codings, and prove the equivalences $(i) \Leftrightarrow(i i i) \Leftrightarrow(i v)$ in Theorem 1 (A) and (B), respectively. The equivalence $(i) \Leftrightarrow($ iii $)$ follows from the following result.

Proposition 3.1. Let $\Phi=\left(E,\left\{f_{i}\right\}_{i=1}^{m}\right) \in \mathcal{E}$ with $I=\operatorname{conv}(E)=[a, b]$.

(i) $f_{1}(I) \cap f_{2}(I) \neq \emptyset$ if and only if $f_{1}(b) \in \mathcal{U}_{\aleph_{0}}(\Phi)$.

(ii) $f_{m-1}(I) \cap f_{m}(I) \neq \emptyset$ if and only if $f_{m}(a) \in \mathcal{U}_{\aleph_{0}}(\Phi)$.

Proof. Since the proofs of (i) and (ii) are similar, we only prove (i).

Suppose $f_{1}(I) \cap f_{2}(I)=\emptyset$. Then in view of the locations of these basic intervals we have

$$
f_{1}(I) \cap f_{i}(I)=\emptyset \quad \text { for any } \quad i \in\{2, \ldots, m\} .
$$

Note that $b=\pi\left(m^{\infty}\right) \in \mathcal{U}_{1}(\Phi)$. Then by (3.1) it follows that $f_{1}(b) \in \mathcal{U}_{1}(\Phi)$. This proves the sufficiency in (i).

For the necessity in (i) we assume $f_{1}(I) \cap f_{2}(I) \neq \emptyset$. Then by Condition (D) there exist $u=u_{1}, v=v_{1} \in \mathbb{N}$ such that $f_{1}(I) \cap f_{2}(I)=f_{1 m^{u}}(I)=f_{21^{v}}(I)$, which gives $f_{1 m^{u}}(\cdot)=f_{21^{v}}(\cdot)$ by Lemma 2.1. Then

$$
f_{1}(b)=\pi\left(1 m^{\infty}\right)=\pi\left(21^{v-1} 1 m^{\infty}\right)=\cdots=\pi\left(\left(21^{v-1}\right)^{k} 1 m^{\infty}\right)=\cdots
$$

for $k=1,2, \ldots$. This implies that $f_{1}(b)$ has at least countably many codings.

In the following we show that $f_{1}(b)$ indeed has countably many codings. Suppose that $\left(d_{i}\right)$ is a coding of $f_{1}(b)$. Note that $f_{1}(b) \in E$ and $f_{1}(b) \in f_{1}(I) \cap f_{2}(I)=f_{1 m^{u}}(I)=$ $f_{21^{v}}(I)$. Then by Lemma 2.2 it follows that $d_{1} \cdots d_{u}=1 m^{u-1}$ or $d_{1} \cdots d_{v}=21^{v-1}$.

- If $d_{1} \cdots d_{u}=1 m^{u-1}$, then by (3.2) we have

$$
\pi\left(d_{u+1} d_{u+2} \cdots\right)=\pi\left(m^{\infty}\right) \in \mathcal{U}_{1}(\Phi)
$$


This implies that $\left(d_{i}\right)=1 m^{\infty}$.

- If $d_{1} \cdots d_{v}=21^{v-1}$, then by (3.2) it yields that

$$
\pi\left(d_{v+1} d_{v+2} \cdots\right)=\pi\left(1 m^{\infty}\right)=f_{1}(b) .
$$

By iteration of the above arguments it follows that all codings of $f_{1}(b)$ are of the form

$$
\left(21^{v-1}\right)^{k} 1 m^{\infty}, \quad k \geq 0 .
$$

Hence, $f_{1}(b) \in \mathcal{U}_{\aleph_{0}}(\Phi)$. This proves the necessity in (i).

Proof of (i) $\Leftrightarrow$ (iii) in Theorem 1 (A) and (B). This follows directly from Proposition 3.1.

In the following it remains to prove the equivalence (i) $\Leftrightarrow$ (iv) in Theorem 1 (A) and (B).

Lemma 3.2. Let $\Phi=\left(E,\left\{f_{i}\right\}_{i=1}^{m}\right) \in \mathcal{E}$ with $I=\operatorname{conv}(E)$. If $f_{1}(I) \cap f_{2}(I)=f_{m-1}(I) \cap$ $f_{m}(I)=\emptyset$, then $\mathcal{U}_{\aleph_{0}}(\Phi)=\emptyset$.

Proof. Suppose on the contrary that $\mathcal{U}_{\aleph_{0}}(\Phi) \neq \emptyset$, and take $x \in \mathcal{U}_{\aleph_{0}}(\Phi)$. Then $x$ must have a coding $\left(d_{i}\right)$ satisfying

$$
x_{n}:=\pi\left(d_{n+1} d_{n+2} \cdots\right) \in E \cap \bigcup_{i=2}^{m-2}\left(f_{i}(I) \cap f_{i+1}(I)\right)
$$

for infinitely many $n \geq 1$.

Take $n$ satisfying (3.3) and assume that $x_{n} \in E \cap f_{i}(I) \cap f_{i+1}(I)$ for some $i \in$ $\{2, \ldots, m-2\}$. By Condition (D) there exist $u=u_{i}, v=v_{i} \in \mathbb{N}$ such that

$$
x_{n}=\pi\left(d_{n+1} d_{n+2} \ldots\right) \in f_{i}(I) \cap f_{i+1}(I)=f_{i m^{u}}(I)=f_{(i+1) 1^{v}}(I) .
$$

Note that $f_{1}(I) \cap f_{2}(I)=f_{m-1}(I) \cap f_{m}(I)=\emptyset$. Then in view of the locations of the basic intervals it follows that

$$
f_{1}(I) \cap f_{j}(I)=f_{\ell}(I) \cap f_{m}(I)=\emptyset
$$

for any $j \neq 1$ and any $\ell \neq m$. Therefore, by (3.4) and Lemma 2.2, and using (3.5) it follows that

$$
d_{n+1} \cdots d_{n+u+1}=i m^{u} \quad \text { or } \quad d_{n+1} \cdots d_{n+v+1}=(i+1) 1^{v} .
$$


Note by (3.4) and Lemma 2.1 that $f_{i m^{u}}(\cdot)=f_{(i+1) 1^{v}}(\cdot)$. Therefore, we have a substitution $i m^{u} \sim(i+1) 1^{v}$ in $d_{n+1} d_{n+2} \cdots$. In other words, there exists a unique $y \in E$ such that $x_{n}=f_{i m^{u}}(y)=f_{(i+1) 1^{v}}(y)$.

Note that (3.3) holds for infinitely many $n \in \mathbb{N}$. Then by an argument similar to the above it follows that there exist infinitely many independent substitutions in $\left(d_{i}\right)$. This implies that $x$ has a continuum of codings, leading to a contradiction with $x \in$ $\mathcal{U}_{\aleph_{0}}(\Phi)$.

Lemma 3.3. Let $\Phi=\left(E,\left\{f_{i}\right\}_{i=1}^{m}\right) \in \mathcal{E}$ with $I=\operatorname{conv}(E)$.

(i) If $f_{1}(I) \cap f_{2}(I)=f_{1 m^{u}}(I)=f_{21^{v}}(I)$ and $f_{m-1}(I) \cap f_{m}(I)=\emptyset$, then $\left|\mathcal{U}_{\aleph_{0}}(\Phi)\right|=$ $\aleph_{0}$. Furthermore, any point in $\mathcal{U}_{\aleph_{0}}(\Phi)$ must have a coding ending with $1 \mathrm{~m}^{\infty} \sim$ $\left(21^{v-1}\right)^{\infty}$

(ii) If $f_{1}(I) \cap f_{2}(I)=\emptyset$ and $f_{m-1}(I) \cap f_{m}(I)=f_{(m-1) m^{p}}(I)=f_{m 1^{q}}(I)$, then $\left|\mathcal{U}_{\aleph_{0}}(\Phi)\right|=$ $\aleph_{0}$. Furthermore, any point in $\mathcal{U}_{\aleph_{0}}(\Phi)$ must have a coding ending with $m 1^{\infty} \sim$ $\left((m-1) m^{p-1}\right)^{\infty}$

(iii) If $f_{1}(I) \cap f_{2}(I)=f_{1 m^{u}}(I)=f_{21^{v}}(I)$ and $f_{m-1}(I) \cap f_{m}(I)=f_{(m-1) m^{p}}(I)=f_{m 1^{q}}(I)$, then $\left|\mathcal{U}_{\aleph_{0}}(\Phi)\right|=\aleph_{0}$. Furthermore, any point in $\mathcal{U}_{\aleph_{0}}(\Phi)$ must have a coding ending with $1 m^{\infty} \sim\left(21^{v-1}\right)^{\infty}$ or $m 1^{\infty} \sim\left((m-1) m^{p-1}\right)^{\infty}$.

Proof. Since the proofs of (ii) and (iii) are similar, we only prove (i). Suppose $f_{1}(I) \cap$ $f_{2}(I) \neq \emptyset$ but $f_{m-1}(I) \cap f_{m}(I)=\emptyset$. By Condition (D) there exist $u=u_{1}, v=v_{1} \in \mathbb{N}$ such that

$$
f_{1}(I) \cap f_{2}(I)=f_{1 m^{u}}(I)=f_{21^{v}}(I)
$$

which yields $f_{1 m^{u}}(\cdot)=f_{21^{v}}(\cdot)$ by Lemma 2.1 .

Denote by $I=[a, b]$ the convex hull of $E$. We claim that $f_{1^{n}}(b) \in \mathcal{U}_{\aleph_{0}}(\Phi)$ for any $n \geq 1$. We will prove this by induction on $n$. Clearly, for $n=1$ we have by Lemma 3.1 that $f_{1}(b) \in \mathcal{U}_{\aleph_{0}}(\Phi)$. Now suppose $f_{1^{n}}(b) \in \mathcal{U}_{\aleph_{0}}(\Phi)$ for some $n \geq 1$, and consider $f_{1^{n+1}}(b)$. If $f_{1^{n+1}}(b) \in f_{2}(I)$, then by Condition (D) it follows that

$$
f_{1^{n+1}}(b) \in f_{1}(I) \cap f_{2}(I) \subseteq f_{1 m}(I)
$$

which implies $f_{1^{n}}(b) \in f_{m}(I)$, leading to a contradiction with $f_{1}(I) \cap f_{m}(I)=\emptyset$. By the induction hypothesis this proves $f_{1^{n+1}}(b) \in \mathcal{U}_{\aleph_{0}}(\Phi)$. Hence, by induction it follows that $\left\{f_{1^{n}}(b): n \geq 1\right\} \subseteq \mathcal{U}_{\aleph_{0}}(\Phi)$.

In the following it suffices to prove that any point in $\mathcal{U}_{\aleph_{0}}(\Phi)$ must have a coding ending with $1 m^{\infty} \sim\left(21^{v-1}\right)^{\infty}$. Take $x \in \mathcal{U}_{\aleph_{0}}(\Phi)$. Suppose on the contrary that $x$ has a coding $\left(d_{i}\right)$ ending with neither $1 m^{\infty}$ nor $\left(21^{v-1}\right)^{\infty}$. Observe that $\left(d_{i}\right)$ satisfies

$$
\pi\left(d_{n+1} d_{n+2} \cdots\right) \in E \cap \bigcup_{i=1}^{m-2}\left(f_{i}(I) \cap f_{i+1}(I)\right)
$$


for infinitely many $n \geq 1$.

Take $n$ satisfying (3.7), and assume $\pi\left(d_{n+1} d_{n+2} \cdots\right) \in f_{i}(I) \cap f_{i+1}(I)$ for some $i \in$ $\{1, \cdots, m-2\}$. Then by Condition (D) there exist $p=u_{i}, q=v_{i} \in \mathbb{N}$ such that

$$
\pi\left(d_{n+1} d_{n+2} \cdots\right) \in f_{i}(I) \cap f_{i+1}(I)=f_{i m^{p}}(I)=f_{(i+1) 1^{q}}(I) .
$$

By Lemma 2.1 it follows that $f_{i m^{p}}(\cdot)=f_{(i+1) 1^{q}}(\cdot)$, and then by Lemma 2.2 we have

$$
d_{n+1} \cdots d_{n+p}=i m^{p-1} \quad \text { or } \quad d_{n+1} \cdots d_{n+q}=(i+1) 1^{q-1} .
$$

Now we split the proof into the following two cases.

Case (I). $d_{n+1} \cdots d_{n+p}=i m^{p-1}$. Then by (3.8) and using $f_{m-1}(I) \cap f_{m}(I)=\emptyset$ it follows that $d_{n+p+1}=m$. Therefore, we have a substitution $d_{n+1} \cdots d_{n+p+1}=i m^{p} \sim$ $(i+1) 1^{q}$.

Case (II). $d_{n+1} \cdots d_{n+q}=(i+1) 1^{q-1}$. Then by (3.8) it follows that $d_{n+q+1}=1$ or 2 .

- If $d_{n+q+1}=1$, then we have a substitution $d_{n+1} \cdots d_{n+q+1}=(i+1) 1^{q} \sim i m^{p}$.

- If $d_{n+q+1}=2$, then by (3.6) and (3.8) it yields that

$$
\pi\left(d_{n+q+1} d_{n+q+2} \cdots\right) \in f_{1}(I) \cap f_{2}(I)=f_{1 m^{u}}(I)=f_{21^{v}}(I) .
$$

So, by Lemma 2.2 it follows that $d_{n+q+1} \cdots d_{n+q+v}=21^{v-1}$ and $d_{n+q+v+1}=1$ or 2 . Note by the assumption that $\left(d_{i}\right)$ does not end with $\left(21^{v-1}\right)^{\infty}$. Then by iteration it follows that there exists $N \geq n+q$ such that

$$
d_{N+1} \cdots d_{N+v+1}=21^{v}
$$

Again, we have a substitution $21^{v} \sim 1 m^{u}$.

By Cases (I)-(II) and (3.7) it follows that there exist infinitely many independent substitutions in $\left(d_{i}\right)$. This implies that $x$ has a continuum of codings, leading to a contradiction with $x \in \mathcal{U}_{\aleph_{0}}(\Phi)$. We complete the proof of (i).

By the proof of Lemma 3.3 it follows that if $f_{1}(I) \cap f_{2}(I) \neq \emptyset$, then any $x \in \mathcal{U}_{\aleph_{0}}(\Phi)$ must have a coding ending with $1 \mathrm{~m}^{\infty}$, which is a coding of $f_{1}(b)$. Similarly, if $f_{m-1}(I) \cap$ $f_{m}(I) \neq \emptyset$, then any $x \in \mathcal{U}_{\aleph_{0}}(\Phi)$ must have a coding ending with $m 1^{\infty}$, which is a coding of $f_{m}(a)$. As a corollary of Lemma 3.3 we have a complete characterization of $\mathcal{U}_{\aleph_{0}}(\Phi)$.

Proposition 3.4. Let $\Phi=\left(E,\left\{f_{i}\right\}_{i=1}^{m}\right) \in \mathcal{E}$ with $I=\operatorname{conv}(E)$. Then

$$
\begin{aligned}
& \mathcal{U}_{\aleph_{0}}(\Phi) \\
& = \begin{cases}\bigcup_{\mathbf{i} \in\{1, \ldots, m\}^{*}}\left\{f_{\mathbf{i}}\left(f_{1}(b)\right)\right\} & \text { if } f_{1}(I) \cap f_{2}(I) \neq \emptyset, f_{m-1}(I) \cap f_{m}(I)=\emptyset ; \\
\bigcup_{\mathbf{i} \in\{1, \ldots, m\}^{*}}\left\{f_{\mathbf{i}}\left(f_{m}(a)\right)\right\} & \text { if } f_{1}(I) \cap f_{2}(I)=\emptyset, f_{m-1}(I) \cap f_{m}(I) \neq \emptyset ; \\
\bigcup_{\mathbf{i} \in\{1, \ldots, m\}^{*}}\left\{f_{\mathbf{i}}\left(f_{1}(b)\right), f_{\mathbf{i}}\left(f_{m}(a)\right)\right\} & \text { if } f_{1}(I) \cap f_{2}(I) \neq \emptyset, f_{m-1}(I) \cap f_{m}(I) \neq \emptyset .\end{cases}
\end{aligned}
$$


Proof of (i) $\Leftrightarrow$ (iv) in Theorem 1 (A) and (B). This follows directly from Lemmas 3.2 and 3.3.

\section{Topology of $\mathcal{U}_{k}(\Phi)$}

In this section we investigate the topology of $\mathcal{U}_{k}(\Phi)$, and prove the equivalence (i) $\Leftrightarrow$ (v) in Theorem 1 (A) and (B) respectively. First we prove the following useful lemma.

Lemma 4.1. Let $\Phi=\left(E,\left\{f_{i}\right\}_{i=1}^{m}\right) \in \mathcal{E}$ with $I=\operatorname{conv}(E)$.

(i) If $f_{1}(I) \cap f_{2}(I)=f_{1 m^{u}}(I)=f_{21^{v}}(I)$ and $f_{i_{0}}(I) \cap f_{i_{0}+1}(I)=\emptyset$ for some $i_{0} \in$ $\{2, \ldots, m-1\}$, then for any integer $\ell \geq 0$

$$
\pi\left(i_{0} m\left(21^{v-1}\right)^{\ell} \mathbf{c}\right) \in \mathcal{U}_{1}(\Phi)
$$

where $\pi(\mathbf{c}) \in f_{i_{0}+1}(E) \cap \mathcal{U}_{1}(\Phi)$.

(ii) If $f_{m-1}(I) \cap f_{m}(I)=f_{(m-1) m^{u}}(I)=f_{m 1^{v}}(I)$ and $f_{i_{0}}(I) \cap f_{i_{0}+1}(I)=\emptyset$ for some $i_{0} \in\{1, \ldots, m-2\}$, then for any integer $\ell \geq 0$

$$
\pi\left(\left(i_{0}+1\right) 1\left((m-1) m^{u-1}\right)^{\ell} \mathbf{c}\right) \in \mathcal{U}_{1}(\Phi)
$$

where $\pi(\mathbf{c}) \in f_{i_{0}}(E) \cap \mathcal{U}_{1}(\Phi)$.

Proof. Since the proof of (ii) is similar, we only prove (i).

Let $x_{\ell}:=\pi\left(i_{0} m\left(21^{v-1}\right)^{\ell} \mathbf{c}\right)$. We will prove $x_{\ell} \in \mathcal{U}_{1}(\Phi)$ by induction on $\ell$. First we prove $x_{0}=\pi\left(i_{0} m \mathbf{c}\right) \in \mathcal{U}_{1}(\Phi)$. Note that $f_{i_{0}}(I) \cap f_{i_{0}+1}(I)=\emptyset$. Then in view of the location of the basic intervals it follows that

$$
f_{i}(I) \cap f_{j}(I)=\emptyset \quad \forall i \leq i_{0} \text { and } j \geq i_{0}+1
$$

Let $\left(d_{i}\right)$ be a coding of $x_{0}$. We will prove $\left(d_{i}\right)=i_{0} m \mathbf{c}$, where $\mathbf{c} \in \pi^{-1}\left(f_{i_{0}+1}(E) \cap \mathcal{U}_{1}(\Phi)\right)$ has a prefix $i_{0}+1$. Since $\pi(\mathbf{c}) \in \mathcal{U}_{1}(\Phi)$, it suffices to prove $d_{1}=i_{0}$ and $d_{2}=m$.

If $d_{1} \neq i_{0}$, then by (4.1) we must have $d_{1}=i_{0}-1$. By Condition (D) this implies

$$
\pi\left(i_{0} m \mathbf{c}\right) \in f_{i_{0}-1}(I) \cap f_{i_{0}}(I) \subset f_{i_{0} 1}(I),
$$

which gives $\pi(m \mathbf{c}) \in f_{1}(I) \cap f_{m}(I)$, leading to a contradiction with (4.1). So $d_{1}=i_{0}$. To determine $d_{2}$ suppose on the contrary $d_{2} \neq m$. Then $d_{2}$ must be $m-1$. By Condition (D) it follows that

$$
\pi(m \mathbf{c}) \in f_{m-1}(I) \cap f_{m}(I) \subset f_{m 1}(I) .
$$


This together with $\pi(\mathbf{c}) \in f_{i_{0}+1}(E)$ yields $\pi(\mathbf{c}) \in f_{1}(I) \cap f_{i_{0}+1}(I)$, again leading to a contradiction with (4.1). Therefore, $\left(d_{i}\right)=i_{0} m \mathbf{c}$ is the unique coding of $x_{0}$, and then $x_{0} \in \mathcal{U}_{1}(\Phi)$.

We proceed with the induction hypothesis that $x_{\ell}=\pi\left(i_{0} m\left(21^{v-1}\right)^{\ell} \mathbf{c}\right) \in \mathcal{U}_{1}(\Phi)$ for some $\ell \geq 0$, and we consider $x_{\ell+1}$. By the induction hypothesis it follows that

$$
\pi\left(\left(21^{v-1}\right)^{\ell} \mathbf{c}\right) \in \mathcal{U}_{1}(\Phi)
$$

Let $\left(d_{i}\right)$ be a coding of $x_{\ell+1}$. By (4.2) it suffices to prove $d_{1} \ldots d_{v+2}=i_{0} m 21^{v-1}$. By an argument similar to the one in the proof of $x_{0} \in \mathcal{U}_{1}(\Phi)$ it follows that $d_{1}=i_{0}$. To determine the second digit $d_{2}$ we assume on the contrary that $d_{2} \neq m$. Then $d_{2}=m-1$, which implies $\pi\left(m\left(21^{v-1}\right)^{\ell+1} \mathbf{c}\right) \in f_{m-1}(I) \cap f_{m}(I) \subset f_{m 1}(I)$. So

$$
\pi\left(\left(21^{v-1}\right)^{\ell+1} \mathbf{c}\right) \in f_{1}(I) \cap f_{2}(I)=f_{21^{v}}(I) .
$$

If $\ell \geq 1$, then (4.3) implies

$$
\pi\left(\left(21^{v-1}\right)^{\ell} \mathbf{c}\right) \in f_{1}(I) \cap f_{2}(I)
$$

leading to a contradiction with (4.2). If $\ell=0$, then (4.3) implies $\pi(\mathbf{c}) \in f_{1}(I) \cap f_{i_{0}+1}(I)$, leading to a contradiction with (4.1). Therefore, $d_{2}=m$.

To prove $d_{3}=2$ we consider the following two cases.

- If $d_{3}=1$, then $\pi\left(\left(21^{v-1}\right)^{\ell+1} \mathbf{c}\right) \in f_{1}(I) \cap f_{2}(I)=f_{21^{v}}(I)$. By the above arguments this will lead to a contradiction.

- If $d_{3}=3$, then $\pi\left(\left(21^{v-1}\right)^{\ell+1} \mathbf{c}\right) \in f_{2}(I) \cap f_{3}(I) \subset f_{2 m}(I)$, which gives $\pi\left(1^{v-1}\left(21^{v-1}\right)^{\ell} \mathbf{c}\right)$ $\in f_{m}(I)$. Again this leads to a contradiction with (4.1).

Therefore, $d_{3}=2$. If $v=1$, then by the induction hypothesis we are done.

If $v>1$, then we claim $d_{4} \ldots d_{v+2}=1^{v-1}$. Suppose $d_{4} \neq 1$. Then $\pi\left(1^{v-1}\left(21^{v-1}\right)^{\ell} \mathbf{c}\right) \in$ $f_{1}(I) \cap f_{2}(I) \subset f_{1 m}(I)$, which leads to a contradiction with (4.1). By iteration of these arguments we can deduce that $d_{4}=\cdots=d_{v+2}=1$.

Hence, $d_{1} \ldots d_{v+2}=i_{0} m 21^{v-1}$. By the induction hypothesis we conclude that $\left(d_{i}\right)=$ $i m\left(21^{v-1}\right)^{\ell+1} \mathbf{c}$, and thus $x_{\ell+1} \in \mathcal{U}_{1}(\Phi)$, completing the proof.

Proposition 4.2. Let $\Phi=\left(E,\left\{f_{i}\right\}_{i=1}^{m}\right) \in \mathcal{E}$ with $I=\operatorname{conv}(E)$. If $f_{1}(I) \cap f_{2}(I) \neq \emptyset$ or $f_{m-1}(I) \cap f_{m}(I) \neq \emptyset$, then $\mathcal{U}_{k}(\Phi)$ is not closed for any $k \in \mathbb{N}$.

Proof. Take $k \in \mathbb{N}$. We will prove that $\mathcal{U}_{k}(\Phi)$ is not closed by considering the following two cases.

(I). $f_{1}(I) \cap f_{2}(I) \neq \emptyset$. By Condition (D) there exist $u=u_{1}, v=v_{1} \in \mathbb{N}$ such that $f_{1}(I) \cap f_{2}(I)=f_{1 m^{u}}(I)=f_{21^{v}}(I)$. Furthermore, by Condition $(\mathrm{C})$ there exists $i_{0} \in\{2, \ldots, m-1\}$ such that $f_{i_{0}}(I) \cap f_{i_{0}+1}(I)=\emptyset$. We claim that for any $\ell \in \mathbb{N}$ 


$$
x_{k, \ell}:=\pi\left(1 m^{u(k-1)} i_{0} m\left(21^{v-1}\right)^{\ell} \mathbf{c}\right) \in \mathcal{U}_{k}(\Phi)
$$

where $\pi(\mathbf{c}) \in f_{i_{0}+1}(E) \cap \mathcal{U}_{1}(\Phi)$. By Lemma 4.1 (i) it follows that $\pi\left(i_{0} m\left(21^{v-1}\right)^{\ell} \mathbf{c}\right) \in$ $f_{i_{0}}(E) \cap \mathcal{U}_{1}(\Phi)$. Then the claim follows by Lemma 2.5 (i).

Observe that $x_{k, \ell} \in \mathcal{U}_{k}(\Phi)$ converges to

$$
x_{k}=\lim _{\ell \rightarrow \infty} z_{k, \ell}=\pi\left(1 m^{u(k-1)} i_{0} m\left(21^{v-1}\right)^{\infty}\right),
$$

which has a coding ending with $\left(21^{v-1}\right)^{\infty}$. So by Proposition 3.4 it follows that $x_{k} \in$ $\mathcal{U}_{\aleph_{0}}(\Phi)$. This implies that $\mathcal{U}_{k}(\Phi)$ is not closed.

(II). $f_{m-1}(I) \cap f_{m}(I) \neq \emptyset$. Then there exist $u=u_{m-1}, v=v_{m-1} \in \mathbb{N}$ such that $f_{m-1}(I) \cap f_{m}(I)=f_{(m-1) m^{u}}(I)=f_{m 1^{v}}(I)$. Furthermore, by Condition (C) there exists $i_{0} \in\{1, \ldots, m-2\}$ such that $f_{i_{0}}(I) \cap f_{i_{0}+1}(I)=\emptyset$. By Lemmas 2.5 (ii) and 4.1 (ii) it follows that

$$
y_{k, \ell}:=\pi\left(m 1^{v(k-1)}\left(i_{0}+1\right) 1\left((m-1) m^{u-1}\right)^{\ell} \mathbf{c}\right) \in \mathcal{U}_{k}(\Phi)
$$

where $\pi(\mathbf{c}) \in f_{i_{0}}(E) \cap \mathcal{U}_{1}(\Phi)$. However, the limit

$$
y_{k}=\lim _{\ell \rightarrow \infty} y_{k, \ell}=\pi\left(m 1^{v(k-1)}\left(i_{0}+1\right) 1\left((m-1) m^{u-1}\right)^{\infty}\right)
$$

has a coding ending with $\left((m-1) m^{u-1}\right)^{\infty} \sim m 1^{\infty}$. So by Proposition 3.4 it follows that $y_{k} \in \mathcal{U}_{\aleph_{0}}(\Phi)$. Therefore, $\mathcal{U}_{k}(\Phi)$ is not closed.

Now we consider the closeness of $\mathcal{U}_{k}(\Phi)$ by assuming $f_{1}(I) \cap f_{2}(I)=f_{m-1}(I) \cap f_{m}(I)=$ $\emptyset$. Note by Lemma 2.7 that $\mathcal{U}_{k}(\Phi)=\emptyset$ if $k \neq 2^{\ell}$ with $\ell \in \mathbb{N} \cup\{0\}$.

Lemma 4.3. Let $\Phi=\left(E,\left\{f_{i}\right\}_{i=1}^{m}\right) \in \mathcal{E}$ with $I=\operatorname{conv}(E)$. If $f_{1}(I) \cap f_{2}(I)=f_{m-1}(I) \cap$ $f_{m}(I)=\emptyset$ and $f_{i}(I) \cap f_{i+1}(I)=f_{i m^{u}}(I)=f_{(i+1) 1^{v}}(I)$ for some $i \in\{2, \ldots, m-2\}$, then

$$
\pi\left(1^{n}\left(i m^{u}\right)^{\ell} 1^{\infty}\right) \in \mathcal{U}_{2^{\ell}}(\Phi)
$$

for any integers $n, \ell \geq 0$.

Proof. Since $f_{1}(I) \cap f_{2}(I)=\emptyset$, it suffices to prove

$$
x_{\ell}:=\pi\left(1\left(i m^{u}\right)^{\ell} 1^{\infty}\right) \in \mathcal{U}_{2^{\ell}}(\Phi) .
$$

We prove this by induction on $\ell$. Clearly, for $\ell=0$ we have $x_{0}=\pi\left(1^{\infty}\right)=a \in \mathcal{U}_{1}(\Phi)$. Now suppose $x_{\ell} \in \mathcal{U}_{2^{\ell}}(\Phi)$ for some $\ell \geq 0$, and we will prove $x_{\ell+1} \in \mathcal{U}_{2^{\ell+1}}(\Phi)$.

Since $f_{1}(I) \cap f_{2}(I)=\emptyset$, by using $\pi\left(1\left(\mathrm{im}^{u}\right)^{\ell} 1^{\infty}\right)=x_{\ell} \in \mathcal{U}_{2^{\ell}}(\Phi)$ it follows that $\pi\left(\left(i m^{u}\right)^{\ell} 1^{\infty}\right) \in \mathcal{U}_{2^{\ell}}(\Phi)$, and then by using $f_{m-1}(I) \cap f_{m}(I)=\emptyset$ we have 


$$
\pi\left(m\left(i m^{u}\right)^{\ell} 1^{\infty}\right) \in \mathcal{U}_{2^{\ell}}(\Phi)
$$

Let $\left(d_{i}\right)$ be a coding of $x_{\ell+1}=\pi\left(1\left(i m^{u}\right)^{\ell+1} 1^{\infty}\right)$. Since $f_{1}(I) \cap f_{2}(I)=\emptyset$, it follows that $d_{1}=1$. For the second digit $d_{2}$ we claim that $d_{2} \in\{i, i+1\}$.

If $d_{2} \notin\{i, i+1\}$, then by using $\pi\left(d_{2} d_{3} \ldots\right) \in f_{i}(I)$ we must have $d_{2}=i-1$. So $\pi\left(\left(i m^{u}\right)^{\ell+1} 1^{\infty}\right) \in f_{i-1}(I) \cap f_{i}(I) \subset f_{i 1}(I)$, which implies

$$
\pi\left(m^{u}\left(i m^{u}\right)^{\ell} 1^{\infty}\right) \in f_{1}(I)
$$

leading to a contradiction with $f_{1}(I) \cap f_{m}(I)=\emptyset$. Therefore, $d_{2}=i$ or $d_{2}=i+1$.

By the claim it follows that

$$
\pi\left(d_{2} d_{3} \ldots\right)=\pi\left(\left(i m^{u}\right)^{\ell+1} 1^{\infty}\right) \in f_{i}(I) \cap f_{i+1}(I)=f_{i m^{u}}(I)=f_{(i+1) 1^{v}}(I) .
$$

This together with Lemma 2.2 implies that either $d_{2} \ldots d_{u+1}=i m^{u-1}$ or $d_{2} \ldots d_{v+1}=$ $(i+1) 1^{v-1}$. Observe that

$$
\pi\left(i m^{u-1} m\left(i m^{u}\right)^{\ell} 1^{\infty}\right)=\pi\left((i+1) 1^{v-1} 1\left(i m^{u}\right)^{\ell} 1^{\infty}\right)
$$

where we have used $f_{i m^{u}}=f_{(i+1) 1^{v}}$ by Lemma 2.1. Then by (4.4) and the induction hypothesis $\pi\left(1\left(\mathrm{im}^{u}\right)^{\ell} 1^{\infty}\right)=x_{\ell} \in \mathcal{U}_{2^{\ell}}(\Phi)$ it follows that $\pi\left(d_{2} d_{3} \ldots\right)=\pi\left(\left(\mathrm{im}^{u}\right)^{\ell+1} 1^{\infty}\right)$ has precisely $2^{\ell}+2^{\ell}=2^{\ell+1}$ different codings. So $x_{\ell+1} \in \mathcal{U}_{2^{\ell+1}}(\Phi)$. By induction this completes the proof.

Given $\delta>0$, for a subset $F$ of $\mathbb{R}$ we define its $\delta$-neighborhood by

$$
F_{\delta}:=\{x \in \mathbb{R}:|x-y|<\delta \text { for some } y \in F\}
$$

In the following proposition we show that $\mathcal{U}_{1}(\Phi)$ is closed by assuming $f_{1}(I) \cap f_{2}(I)=$ $f_{m-1}(I) \cap f_{m}(I)=\emptyset$. To prove this we need the following lemma.

Lemma 4.4. Let $\Phi=\left(E,\left\{f_{i}\right\}_{i=1}^{m}\right) \in \mathcal{E}$ with $I=\operatorname{conv}(E)$. If $f_{i}(I) \cap f_{i+1}(I)=f_{i m^{u}}(I)=$ $f_{(i+1) 1^{v}}(E)$ for some $i \in\{1, \ldots, m-1\}$, then

$$
f_{i}(I) \cap f_{i+1}(I) \cap E=f_{i}(E) \cap f_{i+1}(E)=f_{i m^{u}}(E)=f_{(i+1) 1^{v}}(E) .
$$

Proof. By Lemma 2.1 we have $f_{i m^{u}}=f_{(i+1) 1^{v}}$, and then $f_{i m^{u}}(E)=f_{(i+1) 1^{v}}(E)$. Clearly, $f_{i m^{u}}(E)=f_{(i+1) 1^{v}}(E) \subset f_{i}(E) \cap f_{i+1}(E) \subset f_{i}(I) \cap f_{i+1}(I) \cap E$. So it suffices to prove

$$
f_{i}(I) \cap f_{i+1}(I) \cap E \subset f_{i m^{u}}(E) .
$$

Take $x \in f_{i}(I) \cap f_{i+1}(I) \cap E$. By Lemma 2.3 it follows that $x$ has a coding $\left(d_{i}\right)$ beginning either with $i m^{u-1}$ or with $(i+1) 1^{v-1}$. Since $f_{i}(I) \cap f_{i+1}(I)=f_{i m^{u}}(I)=f_{(i+1) 1^{v}}(I)$, this gives 
$\pi\left(i m^{u-1} d_{u+1} d_{u+2} \ldots\right) \in f_{i m^{u}}(I) \cap E \quad$ or $\quad \pi\left((i+1) 1^{v-1} d_{v+1} d_{v+2} \ldots\right) \in f_{(i+1) 1^{v}}(I) \cap E$.

Whence,

$$
\pi\left(d_{u+1} d_{u+2} \ldots\right) \in f_{m}(I) \cap E \quad \text { or } \quad \pi\left(d_{v+1} d_{v+2} \ldots\right) \in f_{1}(I) \cap E .
$$

Therefore, to establish (4.5) it suffices to prove the following claim.

Claim: $f_{m}(I) \cap E=f_{m}(E)$ and $f_{1}(I) \cap E=f_{1}(E)$.

Since the proof for the second equality is similar, we only prove the first equality $f_{m}(I) \cap E=f_{m}(E)$. If $f_{m-1}(I) \cap f_{m}(I)=\emptyset$, then it is easy to verify $f_{m}(I) \cap E=f_{m}(E)$. Now suppose $f_{m-1}(I) \cap f_{m}(I) \neq \emptyset$. Then there exist positive integers $p, q$ such that $f_{m-1}(I) \cap f_{m}(I)=f_{(m-1) m^{p}}(I)=f_{m 1^{q}}(I)$. It is clear that $f_{m}(E) \subset f_{m}(I) \cap E$. To prove that this is indeed an equality let us assume for some $y \in\left(f_{m}(I) \cap E\right) \backslash f_{m}(E)$. Then by Lemma $2.3 y$ must have a coding $\left(s_{i}\right)$ beginning with $s_{1} \ldots s_{p}=(m-1) m^{p-1}$. Since $y \notin f_{m}(E)$, we must have $s_{p+1}=m-1$, for otherwise we will have a substitution $(m-1) m^{p} \sim m 1^{q}$ which leads to $y \in f_{m}(E)$. Furthermore, $\pi\left(s_{p+1} s_{p+2} \ldots\right) \in f_{m-1}(I) \cap$ $f_{m}(I) \cap E$. By the same argument we can also deduce that

$$
\begin{aligned}
& s_{p+1} \ldots s_{2 p}=(m-1) m^{p-1}, \quad s_{2 p+1}=m-1 \text { and } \\
& \pi\left(s_{2 p+1} s_{2 p+2} \ldots\right) \in f_{m-1}(I) \cap f_{m}(I) \cap E .
\end{aligned}
$$

Iterating this argument we conclude that $y$ has a coding

$$
s_{1} s_{2} \ldots=\left((m-1) m^{p-1}\right)^{\infty} .
$$

By Lemma 3.3 it follows that $y$ also has a coding $m 1^{\infty}$, which implies $y \in f_{m}(E)$, leading to a contradiction. Hence, the equality $f_{m}(I) \cap E=f_{m}(E)$ holds.

Proposition 4.5. Let $\Phi=\left(E,\left\{f_{i}\right\}_{i=1}^{m}\right) \in \mathcal{E}$ with $I=\operatorname{conv}(E)$. If $f_{1}(I) \cap f_{2}(I)=f_{m-1}(I) \cap$ $f_{m}(I)=\emptyset$, then $\mathcal{U}_{k}(\Phi)$ is closed if and only if $k \neq 2^{\ell}$ with $\ell \in \mathbb{N}$. In particular, $\mathcal{U}_{1}(\Phi)$ is closed.

Proof. First we prove the necessity. Suppose $k=2^{\ell}$ for some integer $\ell \geq 1$. By Conditions $(\mathrm{C})$ and $(\mathrm{D})$ there exists $i \in\{2, \ldots, m-2\}$ such that $f_{i}(I) \cap f_{i+1}(I)=f_{i m^{u}}(I)=$ $f_{(i+1) 1^{v}}(I)$. Then by Lemma 4.3 it follows that

$$
x_{n}:=\pi\left(1^{n}\left(i m^{u}\right)^{\ell} 1^{\infty}\right) \in \mathcal{U}_{k}(\Phi) \quad \forall n \in \mathbb{N}
$$

However, the limit $x_{\infty}:=\lim _{n \rightarrow \infty} x_{n}=\pi\left(1^{\infty}\right)=a \in \mathcal{U}_{1}(\Phi)$. So, $\mathcal{U}_{k}(\Phi)$ is not closed for any $k=2^{\ell}$ with $\ell \in \mathbb{N}$.

To prove the sufficiency, in view of Lemma 2.7, it suffices to prove that $\mathcal{U}_{1}(\Phi)$ is closed. Let $T: \bigcup_{i=1}^{m} f_{i}(I) \rightarrow I$ be the inverse map of the IFS $\left\{f_{i}\right\}_{i=1}^{m}$, i.e., 


$$
T(x)=f_{i}^{-1}(x) \quad \text { if } \quad x \in f_{i}(I)
$$

Then $T$ is a multivalued map satisfying $E=T(E)$. So, if $x$ has a coding $\left(d_{i}\right) \in$ $\{1, \ldots, m\}^{\mathbb{N}}$, then $T(x)$ has a coding $\sigma\left(\left(d_{i}\right)\right)=\left(d_{i+1}\right)$, where $\sigma$ is the left-shift map. Note that $f_{1}(I) \cap f_{2}(I)=f_{m-1}(I) \cap f_{m}(I)=\emptyset$. Then

$$
g:=\min \left\{\operatorname{dist}\left(f_{1}(I), f_{2}(I)\right), \operatorname{dist}\left(f_{m-1}(I), f_{m}(I)\right)\right\}>0 .
$$

By Condition (D) it follows that if $f_{i}(I) \cap f_{i+1}(I) \neq \emptyset$, then there exist $u_{i}, v_{i} \in \mathbb{N}$ such that $f_{i}(I) \cap f_{i+1}(I)=f_{i m^{u_{i}}}(I)=f_{(i+1) 1^{v_{i}}}(I)$. Let

$$
\delta:=\min \left\{r_{i} r_{m}^{u_{i}} g: f_{i}(I) \cap f_{i+1}(I) \neq \emptyset\right\}>0 .
$$

Now we claim that

$$
\mathcal{U}_{1}(\Phi)=\left\{x \in E: T^{n}(x) \notin O_{\delta} \forall n \geq 0\right\}
$$

where $O_{\delta}$ is the $\delta$-neighborhood of $O:=\bigcup_{i=1}^{m-1}\left(f_{i}(I) \cap f_{i+1}(I)\right)$.

First we prove the inclusion ' $\supset$ ' in (4.6). Take $x \notin \mathcal{U}_{1}(\Phi)$. Then $x$ has (at least) two different codings $\left(d_{i}\right),\left(d_{i}^{\prime}\right)$ such that

$$
d_{1} \ldots d_{N}=d_{1}^{\prime} \ldots d_{N}^{\prime} \quad \text { and } \quad\left|d_{N+1}-d_{N+1}^{\prime}\right|=1
$$

for some integer $N \geq 0$. This implies $T^{N}(x) \in f_{d_{N+1}}(I) \cap f_{d_{N+1}^{\prime}}(I) \subset O_{\delta}$, and then establishes the inclusion ' $\supset$ '.

To prove the inverse inclusion in (4.6) it suffices to prove that any $x \in O_{\delta} \cap E$ has at least two different codings. By the definition of $\delta$ it follows that

$$
O_{\delta} \cap E=O \cap E
$$

Take $x \in O \cap E$. Then $x \in f_{i}(I) \cap f_{i+1}(I) \cap E$ for some $i \in\{2, \ldots, m-2\}$. By Condition (D) there exist $u=u_{i}, v=v_{i} \in \mathbb{N}$ such that

$$
f_{i}(I) \cap f_{i+1}(I)=f_{i m^{u}}(I)=f_{(i+1) 1^{v}}(I),
$$

which implies $f_{i m^{u}}=f_{(i+1) 1^{v}}$ by Lemma 2.1. So by Lemma 4.4 any $x \in f_{i}(I) \cap f_{i+1}(I) \cap E$ has at least two different codings: one begins with $i m^{u}$, and the other begins with $(i+1) 1^{v}$. This establishes the claim.

Note that $O_{\delta}$ is the finite union of open intervals, and then it is an open set. By (4.6) it follows that

$$
\mathcal{U}_{1}(\Phi)=E \backslash \bigcup_{n=0}^{\infty} T^{-n}\left(O_{\delta}\right)
$$

and then it is closed. This completes the proof. 
Proof of (i) $\Leftrightarrow(\mathbf{v})$ in Theorem 1 (A) and (B). The equivalence (i) $\Leftrightarrow(v)$ follows by Propositions 4.2 and 4.5 .

\section{Geometrical structure of $E$ and $\mathcal{U}_{1}(\Phi)$}

In this section we show that the self-similar set $E$ and the set $\mathcal{U}_{1}(\Phi)$ of points with a unique coding can be both described as the strongly connected graph-directed set satisfying the OSC (see [25]).

\subsection{Geometrical structure of $\mathcal{U}_{1}(\Phi)$}

Given $\Phi=\left(E,\left\{f_{i}\right\}_{i=1}^{m}\right) \in \mathcal{E}$, first we consider the graph-directed structure of the univoque set $\mathcal{U}_{1}(\Phi)$. Let

$$
\mathbf{F}:=\bigcup_{f_{i}(I) \cap f_{i+1}(I)=f_{i m^{u_{i}}}(I)=f_{(i+1) 1} v_{i}(I)}\left\{i m^{u_{i}},(i+1) 1^{v_{i}}\right\}
$$

be the set of forbidden blocks, and let

$$
X_{\mathbf{F}}:=\left\{\left(d_{i}\right) \in\{1, \ldots, m\}^{\mathbb{N}}:\left(d_{i}\right) \text { does not contain any block from } \mathbf{F}\right\}
$$

Then $\left(X_{\mathbf{F}}, \sigma\right)$ is a $N$-step subshift of finite type (cf. [24]), where $N+1$ is the length of the longest word in $\mathbf{F}$, and $\sigma$ is the left shift map. For $n \in \mathbb{N} \cup\{0\}$ we denote by $B_{n}\left(X_{\mathbf{F}}\right)$ the set of all length $n$ admissible words in $X_{\mathbf{F}}$, i.e.,

$$
B_{n}\left(X_{\mathbf{F}}\right)=\left\{\mathbf{d}=d_{1} \ldots d_{n}: \mathbf{d} \text { occurs in some sequence of } X_{\mathbf{F}}\right\}
$$

In particular, for $n=0$ the set $B_{0}\left(X_{\mathbf{F}}\right)$ is the singleton consisting of the empty word $\epsilon$. We will show that $\left(X_{\mathbf{F}}, \sigma\right)$ is transitive, which means any two admissible words can be connected in $X_{\mathbf{F}}$.

Now we construct a directed graph $\mathcal{G}=\left(B_{N}\left(X_{\mathbf{F}}\right), \mathbf{E}\right)$ for the set $\mathcal{U}_{1}(\Phi)$. Let $B_{N}\left(X_{\mathbf{F}}\right)$ be the set of vertices of our graph $\mathcal{G}$. So each admissible word of length $N$ in $X_{\mathbf{F}}$ is a vertex of $\mathcal{G}$. For two vertices $\mathbf{c}=c_{1} \ldots c_{N}, \mathbf{d}=d_{1} \ldots d_{N} \in B_{N}\left(X_{\mathbf{F}}\right)$ we draw a directed edge from $\mathbf{c}$ to $\mathbf{d}$, denoted by $\overrightarrow{\mathbf{c d}}$, if

$$
c_{2} \ldots c_{N}=d_{1} \ldots d_{N-1} \quad \text { and } \quad c_{1} \ldots c_{N} d_{N} \in B_{N+1}\left(X_{\mathbf{F}}\right)
$$

In this case the corresponding map for the edge $\overrightarrow{\mathbf{c d}}$ is denoted by $f_{\overrightarrow{\mathbf{c d}}}=f_{c_{1}}$. Let $\mathbf{E}$ be the set of all directed edges in $\mathcal{G}$. We will show that the set $\mathcal{U}_{1}(\Phi)$ can be identical to the graph-directed set satisfying the OSC.

Note that the set $\mathcal{U}_{1}(\Phi)$ is not alway closed (see Proposition 4.2). We define a countable subset $\mathcal{N}$ of $\pi\left(X_{\mathbf{F}}\right)$ which is not included in $\mathcal{U}_{1}(\Phi)$. 
Definition 5.1. The subset $\mathcal{N}$ of $\pi\left(X_{\mathbf{F}}\right)$ is defined as follows:

- If $f_{1}(I) \cap f_{2}(I)=f_{21^{v}}(I)$ and $f_{m-1}(I) \cap f_{m}(I)=\emptyset$, then

$$
\mathcal{N}=\bigcup_{n=0}^{\infty} \bigcup_{\mathbf{d} \in B_{n}\left(X_{\mathbf{F}}\right)}\left\{\pi\left(\mathbf{d}\left(21^{v-1}\right)^{\infty}\right)\right\}
$$

- If $f_{1}(I) \cap f_{2}(I)=\emptyset$ and $f_{m-1}(I) \cap f_{m}(I)=f_{(m-1) m^{p}}(I)$, then

$$
\mathcal{N}=\bigcup_{n=0}^{\infty} \bigcup_{\mathbf{d} \in B_{n}\left(X_{\mathbf{F}}\right)}\left\{\pi\left(\mathbf{d}\left((m-1) m^{p-1}\right)^{\infty}\right)\right\}
$$

- If $f_{1}(I) \cap f_{2}(I)=f_{21^{v}}(I)$ and $f_{m-1}(I) \cap f_{m}(I)=f_{(m-1) m^{p}}(I)$, then

$$
\mathcal{N}=\bigcup_{n=0}^{\infty} \bigcup_{\mathbf{d} \in B_{n}\left(X_{\mathbf{F}}\right)}\left\{\pi\left(\mathbf{d}\left(21^{v-1}\right)^{\infty}\right), \pi\left(\mathbf{d}\left((m-1) m^{p-1}\right)^{\infty}\right)\right\}
$$

- If $f_{1}(I) \cap f_{2}(I)=f_{m-1}(I) \cap f_{m}(I)=\emptyset$, then $\mathcal{N}=\emptyset$.

In fact, by Proposition 3.4 it follows that any point in $\mathcal{N}$ has countably many codings. For each word $\mathbf{c}=c_{1} \ldots c_{N} \in B_{N}\left(X_{\mathbf{F}}\right)$ we set

$$
\mathcal{U}_{\mathbf{c}}:=\left\{\pi\left(\left(d_{i}\right)\right):\left(d_{i}\right) \in X_{\mathbf{F}}, d_{1} \ldots d_{N}=\mathbf{c}\right\}
$$

where $\pi$ is the projection map defined in (1.1). By Lemma 2.1 and our construction of $X_{\mathbf{F}}$ it follows that

$$
\mathcal{U}_{1}(\Phi) \cup \mathcal{N}=\pi\left(X_{\mathbf{F}}\right)=\bigcup_{\mathbf{c} \in B_{N}\left(X_{\mathbf{F}}\right)} \mathcal{U}_{\mathbf{c}}
$$

where the union in the above equation is pairwise disjoint. Furthermore, for each $\mathbf{c} \in$ $B_{N}\left(X_{\mathbf{F}}\right)$ we have

$$
\mathcal{U}_{\mathbf{c}}=\bigcup_{\mathbf{c d} \in \mathbf{E}} f_{\mathbf{c d}}\left(\mathcal{U}_{\mathbf{d}}\right)
$$

One can also verify that the union in (5.2) is pairwise disjoint. Therefore, by (5.1) and (5.2) it follows that up to a countable set $\mathcal{N}$ the set $\mathcal{U}_{1}(\Phi)$ is a graph-directed set satisfying the OSC.

Lemma 5.2. The subshift of finite type $X_{\mathbf{F}}$ is transitive. Or equivalently, the directed graph $\mathcal{G}$ is strongly connected. 
Proof. By our construction it is clear that $X_{\mathbf{F}}$ is a $N$-step subshift of finite type. So it suffices to prove that $X_{\mathbf{F}}$ is transitive.

By Condition (C) there exists $j \in\{1, \ldots, m-1\}$ such that

$$
f_{j}(I) \cap f_{j+1}(I)=\emptyset .
$$

For any two admissible words $\mathbf{c}=c_{1} \ldots c_{p}$ and $\mathbf{d}=d_{1} \ldots d_{q}$ in $X_{\mathbf{F}}$ we will construct a word $\eta$ such that $\mathbf{c} \eta \mathbf{d}$ is still an admissible word in $X_{\mathbf{F}}$. To rephrase it, the word $\mathbf{c} \eta \mathbf{d}$ does not contain any block from $\mathbf{F}$. Note that $X_{\mathbf{F}}$ is a $N$-step subshift of finite type. We prove the transitivity in the following two steps. In the first step we show that $\mathbf{c}$ can be extended to the right which gives an admissible word $\mathbf{c} w$ ending with $m^{N}$; in the second step we show that the word $m^{N}$ can be connected to $\mathbf{d}$ via a word $w^{\prime}$. Then by using [24, Theorem 2.1.8] it follows that $\mathbf{c} w w^{\prime} \mathbf{d}$ is an admissible word in $X_{\mathbf{F}}$.

Step I. We extend the word $\mathbf{c}$ to the right such that the new word $\mathbf{c} w$ is admissible in $X_{\mathbf{F}}$ and ends with $m^{N}$. This will be verified by the following five cases.

(i). $\mathbf{c}=c_{1} \ldots c_{p}$ ends with $i m^{k}$ for some $i \neq m$ and $k \in\{1, \ldots, p-1\}$. If $f_{i}(I) \cap$ $f_{i+1}(I)=\emptyset$, then we can choose $w=m^{N}$, and one can easily check that $\mathbf{c} w m^{\infty} \in X_{\mathbf{F}}$. If $f_{i}(I) \cap f_{i+1}(I) \neq \emptyset$, then there exist $u=u_{i}, v=v_{i} \in \mathbb{N}$ such that

$$
f_{i}(I) \cap f_{i+1}(I)=f_{i m^{u}}(I)=f_{(i+1) 1^{v}}(I) .
$$

Since $\mathbf{c}=c_{1} \ldots c_{p-k-1} i m^{k}$ is an admissible word, we have $k<u$. By (5.3) it follows that

- if $j \neq 1$, then we can take $w=j m^{N}$, and thus $\mathbf{c} w m^{\infty}=c_{1} \ldots c_{p-k-1} i m^{k} j m^{\infty} \in X_{\mathbf{F}}$;

- if $j=1$, then we can choose $w=(j+1) j m^{N}=21 m^{N}$, and thus $\mathbf{c} w m^{\infty}=$ $c_{1} \ldots c_{p-k-1} i m^{k} 21 m^{\infty} \in X_{\mathbf{F}}$.

(ii). $\mathbf{c}=m^{p}$. Then by an argument similar to the proof in Case (i) one can verify that $\mathbf{c} m^{\infty} \in X_{\mathbf{F}}$. So we can take $w=m^{N}$ in this case.

(iii). $\mathbf{c}=c_{1} \ldots c_{p}$ ends with $i 1^{\ell}$ for some $i \neq 1$ and $\ell \in\{1, \ldots, p-1\}$. If $f_{i-1}(I) \cap$ $f_{i}(I)=\emptyset$, then in view of (5.3) we can take

$$
w=\left\{\begin{array}{lll}
(j+1) j m^{N} & \text { if } & j+1 \neq m, \\
j m^{N}=(m-1) m^{N} & \text { if } \quad j+1=m .
\end{array}\right.
$$

Indeed, one can check that $\mathbf{c} w m^{\infty}=c_{1} \ldots c_{p-\ell-1} i 1^{\ell}(j+1) j m^{\infty} \in X_{\mathbf{F}}$ if $j+1 \neq m$, and $\mathbf{c} w m^{\infty}=c_{1} \ldots c_{p-\ell-1} i 1^{\ell}(m-1) m^{\infty} \in X_{\mathbf{F}}$ if $j+1=m$.

If $f_{i-1}(I) \cap f_{i}(I) \neq \emptyset$, then there exist $u=u_{i-1}, v=v_{i-1} \in \mathbb{N}$ such that

$$
f_{i-1}(I) \cap f_{i}(I)=f_{(i-1) m^{u}}(I)=f_{i 1^{v}}(I) .
$$

Since $\mathbf{c}=c_{1} \ldots c_{p-\ell-1} i 1^{\ell}$ is admissible, we have $\ell<v$. Then we can take 


$$
w= \begin{cases}j m^{N} & \text { if } \quad j \neq 1 \\ (j+1) j m^{N}=21 m^{N} & \text { if } \quad j=1\end{cases}
$$

One can also verify that $\mathbf{c} w m^{\infty} \in X_{\mathbf{F}}$.

(iv). $\mathbf{c}=1^{p}$. By an argument similar to the proof in Case (iii) we can take the word $w$ as defined in (5.4). One can check that $\mathbf{c} w m^{\infty} \in X_{\mathbf{F}}$.

(v). $\mathbf{c}=c_{1} \ldots c_{p}$ with $c_{p}=i \notin\{1, m\}$. If $f_{i}(I) \cap f_{i+1}(I)=\emptyset$, then we can take $w=m^{N}$ since $\mathbf{c} w m^{\infty}=c_{1} \ldots c_{p-1} i m^{\infty} \in X_{\mathbf{F}}$. If $f_{i}(I) \cap f_{i+1}(I) \neq \emptyset$, then in view of (5.3) we can take $w$ as defined in (5.4). Again, one can verify that $\mathbf{c} w m^{\infty} \in X_{\mathbf{F}}$.

Step II. There exists a word $w^{\prime}$ such that $m^{N} w^{\prime} \mathbf{d}$ is admissible in $X_{\mathbf{F}}$. Suppose $\left(d_{i}\right) \in$ $X_{\mathbf{F}}$ begins with $\mathbf{d}$. If $d_{1} \neq 1$, then take $w^{\prime}=m$, and one can check that $m^{N} w^{\prime} d_{1} d_{2} \ldots=$ $m^{N+1} d_{1} d_{2} \ldots \in X_{\mathbf{F}}$. If $d_{1}=1$, then we take $w^{\prime}=j+1$, and thus by (5.3) it follows that $m^{N}(j+1) d_{1} d_{2} \ldots \in X_{\mathbf{F}}$.

By Steps I and II it follows that $X_{\mathbf{F}}$ is a transitive subshift of finite type. So the directed graph $\mathcal{G}$ is strongly connected.

By (5.1), (5.2) and Lemma 5.2 it follows that $\mathcal{U}_{1}(\Phi)$ is identical to a strongly connected graph-directed set satisfying the OSC. Then by using [25, Theorem 3] the Hausdorff dimension of $\mathcal{U}_{1}(\Phi)$ can be calculated via the spectral radius of the corresponding adjacency matrix of $\mathcal{G}$, and the corresponding Hausdorff measures of $\mathcal{U}_{1}(\Phi)$ are positive and finite.

Proposition 5.3. Let $\Phi=\left(E,\left\{f_{i}\right\}_{i=1}^{m}\right) \in \mathcal{E}$. Then up to a countable set the univoque set $\mathcal{U}_{1}(\Phi)$ is a strongly connected graph-directed set satisfying the OSC. So, for $s=$ $\operatorname{dim}_{H} \mathcal{U}_{1}(\Phi)$ the s-dimensional Hausdorff measure of $\mathcal{U}_{1}(\Phi)$ is positive and finite.

\subsection{Geometrical structure of $E$}

Similar to the construction of $\mathcal{G}$, we construct a directed graph $\mathcal{G}_{*}$ for the self-similar set $E$. Let

$$
\mathbf{F}_{*}:=\left\{i m^{u_{i}}: f_{i}(I) \cap f_{i+1}(I)=f_{i m^{u_{i}}}(I)=f_{(i+1) 1^{v_{i}}}(I)\right\}
$$

be the set of forbidden blocks, and let

$$
X_{\mathbf{F}_{*}}:=\left\{\left(d_{i}\right) \in\{1, \ldots, m\}^{\mathbb{N}}:\left(d_{i}\right) \text { does not conatin any word from } \mathbf{F}_{*}\right\} .
$$

Then $\left(X_{\mathbf{F}_{*}}, \sigma\right)$ is a $N_{*}$-step subshift of finite type, where $N_{*}+1$ is the length of the longest word in $\mathbf{F}_{*}$.

Now we describe the graph-directed structure of $E$ based on the $N_{*}$-step subshift of finite type $X_{\mathbf{F}_{*}}$. We construct a directed graph $\mathcal{G}_{*}=\left(B_{N_{*}}\left(X_{\mathbf{F}_{*}}\right), \mathbf{E}_{*}\right)$ in the following way. Let $B_{N_{*}}\left(X_{\mathbf{F}_{*}}\right)$ be the set of vertices of our graph $\mathcal{G}_{*}$. For two vertices $\mathbf{c}=c_{1} \ldots c_{N_{*}}$ and $\mathbf{d}=d_{1} \ldots d_{N_{*}} \in B_{N_{*}}\left(X_{\mathbf{F}_{*}}\right)$ we connect a directed edge from $\mathbf{c}$ to $\mathbf{d}$, denoted by $\overrightarrow{\mathbf{c d}}$, if $c_{2} \ldots c_{N_{*}}=d_{1} \ldots d_{N_{*}-1}$ and $c_{1} \ldots c_{N_{*}} d_{N_{*}} \in B_{N_{*}+1}\left(X_{\mathbf{F}_{*}}\right)$. In this case we write the 
corresponding map $f_{\overrightarrow{\mathbf{c d}}}=f_{c_{1}}$. Let $\mathbf{E}_{*}$ be the collection of all directed edges in the graph $\mathcal{G}_{*}$. For each word $\mathbf{c}=c_{1} \ldots c_{N_{*}} \in B_{N_{*}}\left(X_{\mathbf{F}_{*}}\right)$ let

$$
E_{\mathbf{c}}:=\left\{\pi\left(\left(d_{i}\right)\right):\left(d_{i}\right) \in X_{\mathbf{F}_{*}}, d_{1} \ldots d_{N_{*}}=c_{1} \ldots c_{N_{*}}\right\}
$$

Then the self-similar set $E$ can be written as

$$
E=\pi\left(X_{\mathbf{F}_{*}}\right)=\bigcup_{\mathbf{c} \in B_{N_{*}}\left(X_{\mathbf{F}_{*}}\right)} E_{\mathbf{c}} .
$$

By Lemma 2.1 and our construction of $X_{\mathbf{F}_{*}}$ it follows that the union in (5.5) is pairwise disjoint. Furthermore, for each $\mathbf{c} \in B_{N_{*}}\left(X_{\mathbf{F}_{*}}\right)$ we have

$$
E_{\mathbf{c}}=\bigcup_{\overrightarrow{\mathbf{c d}} \in \mathbf{E}_{*}} f_{\mathbf{c d}}\left(E_{\mathbf{d}}\right)
$$

One can also verify that the union in (5.6) is pairwise disjoint. Therefore, by (5.5) and (5.6) it follows that $E$ is a graph-directed set satisfying the OSC which can be represented by the directed graph $\mathcal{G}_{*}$.

By an argument similar to the proof of Lemma 5.2 one can show that the graph $\mathcal{G}_{*}$ is strongly connected.

Lemma 5.4. The subshift of finite type $\left(X_{\mathbf{F}_{*}}, \sigma\right)$ is transitive. Or equivalently, the graph $\mathcal{G}_{*}$ is strongly connected.

Hence, by (5.5), (5.6), Lemma 5.4 and using [25, Theorem 3] it follows that the Hausdorff dimension of $E$ can be calculated via the spectral radius of the adjacency matrix of $\mathcal{G}_{*}$, and then the corresponding Hausdorff measure of $E$ is positive and finite.

Proposition 5.5. Let $\Phi=\left(E,\left\{f_{i}\right\}_{i=1}^{m}\right) \in \mathcal{E}$. Then the set $E$ is a strongly connected graphdirected set satisfying the OSC. So, for $t=\operatorname{dim}_{H} E$ the $t$-dimensional Hausdorff measure of $E$ is positive and finite.

In the next section we will explicitly determine the Hausdorff dimensions of $\mathcal{U}_{1}(\Phi)$ and $E$.

\section{Hausdorff dimensions and Hausdorff measures of $E$ and $\mathcal{U}_{k}(\Phi)$}

Let $\Phi=\left(E,\left\{f_{i}\right\}_{i=1}^{m}\right) \in \mathcal{E}$. In this section we will investigate the Hausdorff dimensions and Hausdorff measures of $E$ and $\mathcal{U}_{k}(\Phi)$, and prove Theorem 2. 


\subsection{Hausdorff dimensions of $E$ and $\mathcal{U}_{k}(\Phi)$}

Recall from Propositions 5.3 and 5.5 that both the self-similar set $E$ and the univoque set $\mathcal{U}_{1}(\Phi)$ can be identical to strongly connected graph-directed sets satisfying the OSC, and thus the corresponding Hausdorff measures of $E$ and $\mathcal{U}_{1}(\Phi)$ are positive and finite. Based on this and by using Bonferroni inequality we are able to determine the explicit formulae for the Hausdorff dimensions of $E$ and $\mathcal{U}_{k}(\Phi)$, respectively. Moreover, we prove that the Hausdorff measure of $\mathcal{U}_{k}(\Phi)$ is infinite for any $k \geq 2$ satisfying $\mathcal{U}_{k}(\Phi) \neq \emptyset$.

Given $\Phi=\left(E,\left\{f_{i}\right\}_{i=1}^{n}\right) \in \mathcal{E}$, by Propositions 5.3 and 5.5 it follows that the corresponding Hausdorff measures of $E$ and $\mathcal{U}_{1}(\Phi)$ are both positive and finite, i.e., for $s:=\operatorname{dim}_{H} \mathcal{U}_{1}(\Phi)$ and $t:=\operatorname{dim}_{H} E$ we have

$$
\mathcal{H}^{s}\left(\mathcal{U}_{1}(\Phi)\right) \in(0, \infty) \quad \text { and } \quad \mathcal{H}^{t}(E) \in(0, \infty)
$$

Furthermore, note that $\mathcal{U}_{1}(\Phi)$ is a proper subset of $E$, and $E$ is a strongly connected graph-directed set satisfying the OSC. Then $\operatorname{dim}_{H} \mathcal{U}_{1}(\Phi)<\operatorname{dim}_{H} E$. Note that

$$
E=\mathcal{U}_{2^{\aleph_{0}}}(\Phi) \cup \mathcal{U}_{\aleph_{0}}(\Phi) \cup \bigcup_{k=1}^{\infty} \mathcal{U}_{k}(\Phi)
$$

So, by (6.1) and Theorem 1 it follows that

$$
\operatorname{dim}_{H} \mathcal{U}_{2^{\aleph_{0}}}(\Phi)=\operatorname{dim}_{H} E, \quad \text { and } \quad \mathcal{H}^{t}\left(\mathcal{U}_{2^{\aleph_{0}}}(\Phi)\right)=\mathcal{H}^{t}(E) \in(0, \infty) .
$$

In the remaining part of this subsection we will focus on explicit formulae for the Hausdorff dimensions of $E$ and $\mathcal{U}_{1}(\Phi)$, respectively. This will be done by the Bonferroni inequality (cf. [9, Exercise 3.12]).

For $\Phi=\left(E,\left\{f_{i}\right\}_{i=1}^{m}\right) \in \mathcal{E}$ we recall from Definition 1.2 that the overlapping vectors $\mathbf{u}=\left(u_{1}, \ldots, u_{m}\right), \mathbf{v}=\left(v_{1}, \ldots, v_{m}\right)$ of $\Phi$ are defined as follows: if $f_{i}(I) \cap f_{i+1}(I) \neq \emptyset$, then there exist $u, v \in \mathbb{N}$ such that $f_{i}(I) \cap f_{i+1}(I)=f_{i m^{u}}(I)=f_{(i+1) 1^{v}}(I)$, and in this case $u_{i}=u$ and $v_{i}=v$. Otherwise, $u_{i}=v_{i}=\infty$. In particular, $u_{m}=v_{m}=\infty$.

Proposition 6.1. Let $\Phi=\left(E,\left\{f_{i}\right\}_{i=1}^{m}\right) \in \mathcal{E}$ with $f_{i}(x)=r_{i} x+b_{i}$ for $1 \leq i \leq m$. Then the Hausdorff dimension $t=\operatorname{dim}_{H} E$ satisfies

$$
\sum_{i=1}^{m} r_{i}^{t}\left(1-r_{m}^{u_{i} t}\right)=1
$$

Proof. Note that $E=\bigcup_{i=1}^{m} f_{i}(E)$, and by Condition (B) that any three basic intervals have empty intersection. Then by Bonferroni inequality it follows that

$$
\mathcal{H}^{t}(E)=\mathcal{H}^{t}\left(\bigcup_{i=1}^{m} f_{i}(E)\right)=\sum_{i=1} \mathcal{H}^{t}\left(f_{i}(E)\right)-\sum_{i=1}^{m-1} \mathcal{H}^{t}\left(f_{i}(E) \cap f_{i+1}(E)\right),
$$


where $t=\operatorname{dim}_{H} E$. Observe by Condition (D) that if $f_{i}(I) \cap f_{i+1}(I) \neq \emptyset$, then $f_{i}(I) \cap$ $f_{i+1}(I)=f_{i m^{u}}(I)$ for some positive integer $u_{i}$. By Lemma 4.4 this also implies

$$
f_{i}(E) \cap f_{i+1}(E)=f_{i m^{u_{i}}}(E)
$$

Since the Hausdorff measure $\mathcal{H}^{t}$ is translation invariant, using the scaling property of the Hausdorff measure in (6.3) it follows that

$$
\begin{aligned}
\mathcal{H}^{t}(E) & =\sum_{i=1}^{m} \mathcal{H}^{t}\left(f_{i}(E)\right)-\sum_{f_{i}(I) \cap f_{i+1}(I) \neq \emptyset} \mathcal{H}^{t}\left(f_{i m^{u_{i}}}(E)\right) \\
& =\sum_{i=1}^{m} r_{i}^{t} \mathcal{H}^{t}(E)-\sum_{f_{i}(I) \cap f_{i+1}(I) \neq \emptyset} r_{i}^{t} r_{m}^{u_{i} t} \mathcal{H}^{t}(E) \\
& =\sum_{i=1}^{m} r_{i}^{t}\left(1-r_{m}^{u_{i} t}\right) \mathcal{H}^{t}(E),
\end{aligned}
$$

where the last equality holds by using that $r_{m}^{u_{i} t}=0$ if $f_{i}(I) \cap f_{i+1}(I)=\emptyset$. Note by $(6.1)$ that $\mathcal{H}^{t}(E) \in(0, \infty)$. Then the lemma follows by dividing $\mathcal{H}^{t}(E)$ on both sides of the above equation.

Now we turn to determine the explicit formula for the Hausdorff dimension of $\mathcal{U}_{1}(\Phi)$. First we need the following lemma. Set $\mathcal{U}:=\mathcal{U}_{1}(\Phi)$, and for a word $\mathbf{w} \in\{1, \ldots, m\}^{*}$ write $\mathcal{U}(\mathbf{w}):=\mathcal{U} \cap f_{\mathbf{w}}(E)$. Then any $x \in \mathcal{U}(\mathbf{w})$ has a unique coding with a prefix $\mathbf{w}$.

Lemma 6.2. Let $\Phi=\left(E,\left\{f_{i}\right\}_{i=1}^{m}\right) \in \mathcal{E}$ with $f_{i}(x)=r_{i} x+b_{i}$ for $1 \leq i \leq m$.

(i) For any $i \in\{1, \ldots, m\}$,

$$
\begin{aligned}
& \mathcal{U}(i)= \\
& \begin{cases}f_{i}(\mathcal{U}) & \text { if } f_{i-1}(I) \cap f_{i}(I)=f_{i}(I) \cap f_{i+1}(I)=\emptyset, \\
f_{i}(\mathcal{U}) \backslash f_{i}\left(\mathcal{U}\left(1^{v_{i-1}}\right)\right) & \text { if } f_{i-1}(I) \cap f_{i}(I) \neq \emptyset \text { and } f_{i}(I) \cap f_{i+1}(I)=\emptyset, \\
f_{i}(\mathcal{U}) \backslash f_{i}\left(\mathcal{U}\left(m^{u_{i}}\right)\right) & \text { if } f_{i-1}(I) \cap f_{i}(I)=\emptyset \text { and } f_{i}(I) \cap f_{i+1}(I) \neq \emptyset, \\
f_{i}(\mathcal{U}) \backslash\left(f_{i}\left(\mathcal{U}\left(1^{v_{i-1}}\right)\right) \cup f_{i}\left(\mathcal{U}\left(m^{u_{i}}\right)\right)\right) & \text { if } f_{i-1}(I) \cap f_{i}(I) \neq \emptyset \text { and } f_{i}(I) \cap f_{i+1}(I) \neq \emptyset .\end{cases}
\end{aligned}
$$

(ii) For any $u, v \in \mathbb{N}$ we have

$$
\mathcal{U}\left(m^{u}\right)= \begin{cases}f_{m^{u}}(\mathcal{U}) & \text { if } \quad f_{m-1}(I) \cap f_{m}(I)=\emptyset \\ f_{m^{u}}(\mathcal{U}) \backslash f_{m^{u}}\left(\mathcal{U}\left(1^{v_{m-1}}\right)\right) & \text { if } \quad f_{m-1}(I) \cap f_{m}(I) \neq \emptyset\end{cases}
$$

and

$$
\mathcal{U}\left(1^{v}\right)=\left\{\begin{array}{lll}
f_{1^{v}}(\mathcal{U}) & \text { if } & f_{1}(I) \cap f_{2}(I)=\emptyset \\
f_{1^{v}}(\mathcal{U}) \backslash f_{1^{v}}\left(\mathcal{U}\left(m^{u_{1}}\right)\right) & \text { if } & f_{1}(I) \cap f_{2}(I) \neq \emptyset
\end{array}\right.
$$


Proof. Let $i \in\{1,2, \ldots, m\}$. Since the proofs for different cases in (i) are similar, we only prove that

$$
\mathcal{U}(i)=f_{i}(\mathcal{U}) \backslash\left(f_{i}\left(\mathcal{U}\left(1^{v_{i-1}}\right)\right) \cup f_{i}\left(\mathcal{U}\left(m^{u_{i}}\right)\right)\right)
$$

assuming that $f_{i-1}(I) \cap f_{i}(I) \neq \emptyset$ and $f_{i}(I) \cap f_{i+1}(I) \neq \emptyset$.

Take $x \in \mathcal{U}(i)$. Then $x$ has a unique coding $\pi^{-1}(x)=x_{1} x_{2} \ldots$ with $x_{1}=i$. Clearly, $\pi\left(x_{2} x_{3} \ldots\right) \in \mathcal{U}$. This implies $x=\pi\left(i x_{2} x_{3} \ldots\right) \in f_{i}(\mathcal{U})$. Since by Condition (D) that $f_{i-1}(I) \cap f_{i}(I)=f_{(i-1) m^{u_{i-1}}}(I)=f_{i 1^{v_{i-1}}}(I)$, any point in $f_{i}\left(\mathcal{U}\left(1^{v_{i-1}}\right)\right)$ has at least two different codings: one begins with $i 1^{v_{i-1}}$ and another begins with $(i-1) m^{u_{i-1}}$. This gives $x \notin f_{i}\left(\mathcal{U}\left(1^{v_{i-1}}\right)\right)$. Similarly, note by Condition (D) that $f_{i}(I) \cap f_{i+1}(I)=$ $f_{i m^{u_{i}}}(I)=f_{(i+1) 1^{v_{i}}}(I)$. Then any point in $f_{i}\left(\mathcal{U}\left(m^{u_{i}}\right)\right)$ has at least two codings: one begins with $i m^{u_{i}}$ and another begins with $(i+1) 1^{v_{i}}$. So, $x \notin f_{i}\left(\mathcal{U}\left(m^{u_{i}}\right)\right)$. This proves $\mathcal{U}(i) \subset f_{i}(\mathcal{U}) \backslash\left(f_{i}\left(\mathcal{U}\left(1^{v_{i-1}}\right)\right) \cup f_{i}\left(\mathcal{U}\left(m^{u_{i}}\right)\right)\right)$.

To prove the reverse inclusion we take $y \in f_{i}(\mathcal{U}) \backslash\left(f_{i}\left(\mathcal{U}\left(1^{v_{i-1}}\right)\right) \cup f_{i}\left(\mathcal{U}\left(m^{u_{i}}\right)\right)\right)$. Then $y$ has a coding $y_{1} y_{2} \ldots$ satisfies

$$
y_{1}=i \quad \text { and } \quad \pi\left(y_{2} y_{3} \ldots\right) \in \mathcal{U} \text {. }
$$

Furthermore,

$$
y_{2} \ldots y_{1+v_{i-1}} \neq 1^{v_{i-1}} \text { and } y_{2} \ldots y_{1+u_{i}} \neq m^{u_{i}}
$$

It remains to prove $y \in \mathcal{U}$. Suppose on the contrary that $y$ has another coding $y_{1}^{\prime} y_{2}^{\prime} \ldots$ If $y_{1}^{\prime}=y_{1}=i$, then

$$
\pi\left(y_{2}^{\prime} y_{3}^{\prime} \ldots\right)=\pi\left(y_{2} y_{3} \ldots\right)
$$

has at least two different codings, leading to a contradiction with (6.4). So $y_{1}^{\prime} \neq y_{1}$. Note that $y_{1}=i$. This implies $y \in f_{i-1}(I) \cap f_{i}(I)$ or $y \in f_{i}(I) \cap f_{i+1}(I)$. If $y \in f_{i-1}(I) \cap f_{i}(I)$, then by (6.4) it follows that $y_{2} \ldots y_{1+v_{i-1}}=1^{v_{i-1}}$, leading to a contradiction with (6.5). If $y \in f_{i}(I) \cap f_{i+1}(I)$, then by (6.4) we obtain $y_{2} \ldots y_{1+u_{i}}=m^{u_{i}}$, again leading to a contradiction with (6.5). Therefore, $y_{1} y_{2} \ldots$ is the unique coding of $y$, i.e., $y \in \mathcal{U}(i)$. This completes the proof.

(ii) can be proved analogously to (i).

Proposition 6.3. Let $\Phi=\left(E,\left\{f_{i}\right\}_{i=1}^{m}\right) \in \mathcal{E}$ with $f_{i}(x)=r_{i} x+b_{i}$ for $1 \leq i \leq m$. Then the Haudorff dimension $s=\operatorname{dim}_{H} \mathcal{U}_{1}(\Phi)$ satisfies

$$
\sum_{i=1}^{m} r_{i}^{s}\left(1-\frac{r_{m}^{u_{i} s}\left(2-r_{1}^{v_{m-1} s}-r_{m}^{u_{1} s}\right)}{1-r_{1}^{v_{m-1} s} r_{m}^{u_{1} s}}\right)=1
$$


Proof. Suppose without loss of generality that $f_{1}(I) \cap f_{2}(I) \neq \emptyset$ and $f_{m-1}(I) \cap f_{m}(I) \neq \emptyset$. Let $\mathcal{U}=\mathcal{U}(\Phi)$, and for a word $\mathbf{w} \in\{1,2, \ldots, m\}^{*}$ we write $\mathcal{U}(\mathbf{w})=\mathcal{U} \cap f_{\mathbf{w}}(E)$. Note that

$$
\mathcal{U}=\bigcup_{i=1}^{m} \mathcal{U}(i)
$$

where the union is pairwise disjoint. By Lemma 6.2 (i) it follows that if $f_{i-1}(I) \cap f_{i}(I) \neq \emptyset$ and $f_{i}(I) \cap f_{i+1}(I) \neq \emptyset$, then $\mathcal{U}(i)$ can be written as

$$
\mathcal{U}(i)=f_{i}(\mathcal{U}) \backslash\left(f_{i}\left(\mathcal{U}\left(1^{v_{i-1}}\right) \cup f_{i}\left(\mathcal{U}\left(m^{u_{i}}\right)\right)\right)\right)
$$

where the union is disjoint. For other cases $\mathcal{U}(i)$ can be written analogously, see Lemma 6.2 (i). Let $s:=\operatorname{dim}_{H} \mathcal{U}$. Then the $s$-dimensional Hausdorff measure of $\mathcal{U}$ can be written as

$$
\begin{aligned}
\mathcal{H}^{s}(\mathcal{U})=\sum_{i=1}^{m} \mathcal{H}^{s}(\mathcal{U}(i)) & =\sum_{i=1}^{m}\left(\mathcal{H}^{s}\left(f_{i}(\mathcal{U})\right)-\mathcal{H}^{s}\left(f_{i}\left(\mathcal{U}\left(1^{v_{i-1}}\right)\right)\right)-\mathcal{H}^{s}\left(f_{i}\left(\mathcal{U}\left(m^{u_{i}}\right)\right)\right)\right) \\
& =\sum_{i=1}^{m} r_{i}^{s}\left(\mathcal{H}^{s}(\mathcal{U})-\mathcal{H}^{s}\left(\mathcal{U}\left(1^{v_{i-1}}\right)\right)-\mathcal{H}^{s}\left(\mathcal{U}\left(m^{u_{i}}\right)\right)\right),
\end{aligned}
$$

where we set $v_{0}:=\infty$. We emphasize that if $f_{i-1}(I) \cap f_{i}(I)=\emptyset$, then $v_{i-1}=\infty$ which implies $\mathcal{H}^{s}\left(\mathcal{U}\left(1^{v_{i-1}}\right)\right)=0$. Similarly, if $f_{i}(I) \cap f_{i+1}(I)=\emptyset$, then $u_{i}=\infty$ which gives $\mathcal{H}^{s}\left(\mathcal{U}\left(m^{u_{i}}\right)\right)=0$. So (6.6) holds for all cases independent of the locations of the basic intervals $f_{i-1}(I), f_{i}(I)$ and $f_{i+1}(I)$.

Since $f_{1}(I) \cap f_{2}(I) \neq \emptyset$ and $f_{m-1}(I) \cap f_{m}(I) \neq \emptyset$, by Lemma 6.2 (ii) it follows that

$$
\begin{aligned}
\mathcal{H}^{s}\left(\mathcal{U}\left(1^{v_{i-1}}\right)\right) & =\mathcal{H}^{s}\left(f_{1^{v_{i-1}}}(\mathcal{U})\right)-\mathcal{H}^{s}\left(f_{1^{v_{i-1}}}\left(\mathcal{U}\left(m^{u_{1}}\right)\right)\right) \\
& =r_{1}^{v_{i-1} s}\left(\mathcal{H}^{s}(\mathcal{U})-\mathcal{H}^{s}\left(\mathcal{U}\left(m^{u_{1}}\right)\right)\right) \\
& =r_{1}^{v_{i-1} s} \mathcal{H}^{s}(\mathcal{U})-r_{1}^{v_{i-1} s}\left(\mathcal{H}^{s}\left(f_{m^{u_{1}}}(\mathcal{U})\right)-\mathcal{H}^{s}\left(f_{m^{u_{1}}}\left(\mathcal{U}\left(1^{v_{m-1}}\right)\right)\right)\right) \\
& =r_{1}^{v_{i-1} s} \mathcal{H}^{s}(\mathcal{U})-r_{1}^{v_{i-1} s} r_{m}^{u_{1} s} \mathcal{H}^{s}(\mathcal{U})+r_{1}^{v_{i-1} s} r_{m}^{u_{1} s} \mathcal{H}^{s}\left(\mathcal{U}\left(1^{v_{m-1}}\right)\right) \\
& =\mathcal{H}^{s}(\mathcal{U}) r_{1}^{v_{i-1} s}\left(1-r_{m}^{u_{1} s}\right)+r_{1}^{v_{i-1} s} r_{m}^{u_{1} s} \mathcal{H}^{s}\left(\mathcal{U}\left(1^{v_{m-1}}\right)\right) .
\end{aligned}
$$

Repeating using Lemma 6.2 (ii) in the above equation we can deduce that for any $N \in \mathbb{N} \cup\{0\}$,

$$
\begin{aligned}
\mathcal{H}^{s}\left(\mathcal{U}\left(1^{v_{i-1}}\right)\right)=\mathcal{H}^{s}(\mathcal{U}) r_{1}^{v_{i-1} s}\left(1-r_{m}^{u_{1} s}\right) \sum_{k=0}^{N}\left(r_{1}^{v_{m-1} s} r_{m}^{u_{1} s}\right)^{k} \\
+r_{1}^{v_{i-1} s} r_{m}^{u_{1} s}\left(r_{1}^{v_{m-1} s} r_{m}^{u_{1} s}\right)^{N} \mathcal{H}^{s}\left(\mathcal{U}\left(1^{v_{m-1}}\right)\right) .
\end{aligned}
$$


Letting $N \rightarrow \infty$, and then $\left(r_{1}^{v_{m-1} s} r_{m}^{u_{1} s}\right)^{N} \rightarrow 0$, using that $\mathcal{H}^{s}\left(\mathcal{U}\left(1^{v_{m-1}}\right)\right) \leq \mathcal{H}^{s}(\mathcal{U})<\infty$ we obtain

$$
\mathcal{H}^{s}\left(\mathcal{U}\left(1^{v_{i-1}}\right)\right)=\mathcal{H}^{s}(\mathcal{U}) \frac{r_{1}^{v_{i-1} s}\left(1-r_{m}^{u_{1} s}\right)}{1-r_{1}^{v_{m-1} s} r_{m}^{u_{1} s}}
$$

Similarly, one can prove

$$
\mathcal{H}^{s}\left(\mathcal{U}\left(m^{u_{i}}\right)\right)=\mathcal{H}^{s}(\mathcal{U}) \frac{r_{m}^{u_{i} s}\left(1-r_{1}^{v_{m-1} s}\right)}{1-r_{1}^{v_{m-1} s} r_{m}^{u_{1} s}}
$$

Substituting (6.7) and (6.8) into (6.6), and using that $\mathcal{H}^{s}(\mathcal{U}) \in(0, \infty)$ it follows that

$$
1=\sum_{i=1}^{m} r_{i}^{s}-\frac{1-r_{m}^{u_{1} s}}{1-r_{1}^{v_{m-1} s} r_{m}^{u_{1} s}} \sum_{i=1}^{m} r_{i}^{s} r_{1}^{v_{i-1} s}-\frac{1-r_{1}^{v_{m-1} s}}{1-r_{1}^{v_{m-1} s} r_{m}^{u_{1} s}} \sum_{i=1}^{m} r_{i}^{s} r_{m}^{u_{i} s}
$$

Note that $v_{0}=\infty, u_{m}=\infty$, and by $f_{i 1^{v_{i-1}}}=f_{(i-1) m^{u_{i-1}}}$ that $r_{i} r_{1}^{v_{i-1}}=r_{i-1} r_{m}^{u_{i-1}}$. Rearranging the second summation in the above equation we conclude that the Hausdorff dimension $s=\operatorname{dim}_{H} \mathcal{U}$ satisfies

$$
\begin{aligned}
& 1=\sum_{i=1}^{m} r_{i}^{s}-\frac{1-r_{m}^{u_{1} s}}{1-r_{1}^{v_{m-1} s} r_{m}^{u_{1} s}} \sum_{i=1}^{m} r_{i}^{s} r_{m}^{u_{i} s}-\frac{1-r_{1}^{v_{m-1} s}}{1-r_{1}^{v_{m-1} s} r_{m}^{u_{1} s}} \sum_{i=1}^{m} r_{i}^{s} r_{m}^{u_{i} s} \\
& =\sum_{i=1}^{m} r_{i}^{s}\left(1-\frac{r_{m}^{u_{i} s}\left(2-r_{1}^{v_{m-1} s}-r_{m}^{u_{1} s}\right)}{1-r_{1}^{v_{m-1} s} r_{m}^{u_{1} s}}\right) \text {. }
\end{aligned}
$$

This completes the proof.

\subsection{Hausdorff measure of $\mathcal{U}_{k}(\Phi)$}

Given $\Phi=\left(E,\left\{f_{i}\right\}_{i=1}^{m}\right) \in \mathcal{E}$, in this subsection we will show that the corresponding Hausdorff measure of $\mathcal{U}_{k}(\Phi)$ is infinite for any $k \geq 2$ satisfying $\mathcal{U}_{k}(\Phi) \neq \emptyset$.

Let $\Phi=\left(E,\left\{f_{i}\right\}_{i=1}^{m}\right) \in \mathcal{E}$. By Lemmas 2.5 and 2.7 we construct a large subset of $\mathcal{U}_{k}(\Phi)$ as described in the following lemma. For simplicity we write $\mathcal{U}:=\mathcal{U}_{1}(\Phi)$ and $\mathbf{U}:=\pi^{-1}(\mathcal{U})$. Furthermore, we denote by $B_{n}(\mathbf{U})$ the set of all length $n$ prefixes of sequences from $\mathbf{U}$.

Lemma 6.4. Let $\Phi=\left(E,\left\{f_{i}\right\}_{i=1}^{m}\right) \in \mathcal{E}$ with $I=\operatorname{conv}(E)$, and let $k \in \mathbb{N}$.

(i) If $f_{1}(I) \cap f_{2}(I)=f_{1 m^{u}}(I)$ and $f_{i}(I) \cap f_{i+1}(I)=\emptyset$ for some $i \in\{2, \ldots, m-1\}$, then

$$
\pi\left(c_{1} \ldots c_{n} 1 m^{u(k-1)} \mathbf{d}\right) \in \mathcal{U}_{k}(\Phi)
$$

for any $c_{1} \ldots c_{n} \in B_{n}(\mathbf{U})$ with $c_{n}=i+1$ and for any $\mathbf{d}=d_{1} d_{2} \ldots \in \mathbf{U}$ with $d_{1}=i$. 
(ii) If $f_{m-1}(I) \cap f_{m}(I)=f_{m 1^{v}}(I)$ and $f_{i}(I) \cap f_{i+1}(I)=\emptyset$ for some $i \in\{1, \ldots, m-2\}$, then

$$
\pi\left(c_{1} \ldots c_{n} m 1^{v(k-1)} \mathbf{d}\right) \in \mathcal{U}_{k}(\Phi)
$$

for any $c_{1} \ldots c_{n} \in B_{n}(\mathbf{U})$ with $c_{n}=i$ and for any $\mathbf{d}=d_{1} d_{2} \ldots \in \mathbf{U}$ with $d_{1}=i+1$.

(iii) If $f_{1}(I) \cap f_{2}(I)=f_{m-1}(I) \cap f_{m}(I)=\emptyset$ and $f_{i}(I) \cap f_{i+1}(I)=f_{\text {im }^{u}}(I)$ for some $i \in\{2, \ldots, m-2\}$, then

$$
\pi\left(c_{1} \ldots c_{n}\left(i m^{u}\right)^{k} \mathbf{d}\right) \in \mathcal{U}_{2^{k}}(\Phi)
$$

for any $c_{1} \ldots c_{n} \in B_{n}(\mathbf{U})$ with $c_{n}=m$ and for any $\mathbf{d}=d_{1} d_{2} \ldots \in \mathbf{U}$ with $d_{1}=m$.

For $\Phi=\left(E,\left\{f_{i}\right\}_{i=1}^{m}\right) \in \mathcal{E}$ note by Proposition 5.3 that $\mathcal{U}_{1}(\Phi)$ is identical to a strongly connected graph-directed set satisfying the OSC. In [25] Mauldin and Williams showed that the Hausdorff dimension $s=\operatorname{dim}_{H} \mathcal{U}_{1}(\Phi)$ can be calculated via the spectral radius of the corresponding adjacency matrix $A(s)=\left(a_{i, j}(s)\right)$ which is defined in the following way. Recall from Section 5 that $\mathcal{U}_{1}(\Phi)$ can be represented by the directed graph $\mathcal{G}=$ $\left(B_{N}\left(X_{\mathbf{F}}\right), \mathbf{E}\right)$. The size of the matrix $A(s)$ is $\left|B_{N}\left(X_{\mathbf{F}}\right)\right| \times\left|B_{N}\left(X_{\mathbf{F}}\right)\right|$. For two vertices $\mathbf{c}=c_{1} \ldots c_{N}$ and $\mathbf{d}=d_{1} \ldots d_{N}$, if $\mathbf{c}$ is connected to $\mathbf{d}$, then we define the map for the edge $\overrightarrow{\mathbf{c d}}$ by $f_{\overrightarrow{\mathbf{c d}}}(x)=f_{c_{1}}(x)$. In this case, the corresponding entry of $A(s)$ is defined by

$$
a_{\mathbf{c}, \mathbf{d}}(s)=r_{c_{1}}^{s}
$$

If $\mathbf{c}$ is not connected to $\mathbf{d}$, then we define $a_{\mathbf{c}, \mathbf{d}}(s)=0$.

Note by Lemma 5.2 that the directed graph $\mathcal{G}$ is strongly connected. Then $A(s)$ is an irreducible non-negative matrix. So it has a unique largest non-negative eigenvalue $\rho(s)$, which is also called the Perron eigenvalue of $A(s)$. It was shown in [25, Theorem 3] that the Hausdorff dimension $s=\operatorname{dim}_{H} \mathcal{U}_{1}(\Phi)$ satisfies

$$
\rho(s)=1
$$

Proposition 6.5. Let $\Phi=\left(E,\left\{f_{i}\right\}_{i=1}^{m}\right) \in \mathcal{E}$ and let $s=\operatorname{dim}_{H} \mathcal{U}_{1}(\Phi)$. Then

$$
\mathcal{H}^{s}\left(\mathcal{U}_{k}(\Phi)\right)=\infty
$$

for any $k \geq 2$ satisfying $\mathcal{U}_{k}(\Phi) \neq \emptyset$.

Proof. Let $I=\operatorname{conv}(E)$ and let $k \geq 2$ with $\mathcal{U}_{k}(\Phi) \neq \emptyset$. Since the proof for different cases is similar, we assume without loss of generality that $f_{1}(I) \cap f_{2}(I) \neq \emptyset$ and $f_{i}(I) \cap$ $f_{i+1}(I)=\emptyset$ for some $i \in\{1, \ldots, m-1\}$. So there exists a positive integer $u$ such that $f_{1}(I) \cap f_{2}(I)=f_{1 m^{u}}(I)$. By Lemma 6.4 (i) it follows that 


$$
\bigcup_{n=1}^{\infty} \bigcup_{c_{1} \ldots c_{n} \in B_{n}(\mathbf{U}), c_{n}=i+1}\left\{\pi\left(c_{1} \ldots c_{n} 1 m^{u(k-1)} d_{1} d_{2} \ldots\right):\left(d_{i}\right) \in \mathbf{U}, d_{1}=i\right\} \subset \mathcal{U}_{k}(\Phi),
$$

where $\mathbf{U}=\pi^{-1}\left(\mathcal{U}_{1}(\Phi)\right)$. Furthermore, note that $k \geq 2, c_{1} \ldots c_{n}$ is an admissible word in $\mathbf{U}$, and $f_{i}(I) \cap f_{i+1}(I)=\emptyset$. Then one can verify that the union in the above equation is pairwise disjoint. Let $s=\operatorname{dim}_{H} \mathcal{U}_{1}(\Phi)$. Therefore,

$$
\begin{aligned}
& \mathcal{H}^{s}\left(\mathcal{U}_{k}(\Phi)\right) \\
& \geq \sum_{n=1}^{\infty} \sum_{c_{1} \ldots c_{n} \in B_{n}(\mathcal{U}), c_{n}=i+1} \mathcal{H}^{s}\left(\left\{\pi\left(c_{1} \ldots c_{n} 1 m^{u(k-1)} d_{1} d_{2} \ldots\right):\left(d_{i}\right) \in \mathbf{U}, d_{1}=i\right\}\right) \\
& \quad=D \sum_{n=1}^{\infty} \sum_{c_{1} \ldots c_{n} \in B_{n}(\mathbf{U}), c_{n}=i+1}\left(\prod_{j=1}^{n} r_{c_{j}}^{s}\right),
\end{aligned}
$$

where

$$
\begin{aligned}
D & :=\mathcal{H}^{s}\left(\left\{\pi\left(1 m^{u(k-1)} d_{1} d_{2} \ldots\right):\left(d_{i}\right) \in \mathbf{U}, d_{1}=i\right\}\right) \\
& =r_{1}^{s} r_{m}^{u(k-1) s} \mathcal{H}^{s}\left(f_{i}(E) \cap \mathcal{U}_{1}(\Phi)\right)>0
\end{aligned}
$$

using Proposition 5.3. Note that $D$ is a constant independent of the summation in (6.9). By using the Perron-Frobenius Theorem (cf. [24, Chapter 4]) it follows that

$$
\sum_{c_{1} \ldots c_{n} \in B_{n}(\mathbf{U}), c_{n}=i+1}\left(\prod_{j=1}^{n} r_{c_{j}}^{s}\right) \geq D_{0} \cdot \rho(s)^{n}
$$

for some constant $D_{0}>0$, where $\rho(s)$ is the Perron eigenvalue of the matrix $\mathbf{A}(s)$. Note that $\rho(s)=1$. By (6.9) and (6.10) we conclude that

$$
\mathcal{H}^{s}\left(\mathcal{U}_{k}(\Phi)\right) \geq \sum_{n=1}^{\infty} D \cdot D_{0}=\infty
$$

Proof of Theorem 2. The Hausdorff dimensions and Hausdorff measures of $E$ and $\mathcal{U}_{2 \aleph_{0}}(\Phi)$ follow by Propositions 5.5 and 6.1. This proves (i). For the Hausdorff dimension of $\mathcal{U}_{k}(\Phi)$ it can be deduced from Theorem 1 and Proposition 6.3. For the Hausdorff measures of $\mathcal{U}_{k}(\Phi)$ it follows from Propositions 5.3 and 6.5. This completes the proof.

\section{Local dimension of self-similar measure}

Let $\Phi=\left(E,\left\{f_{i}\right\}_{i=1}^{m}\right) \in \mathcal{E}$. Given a probability vector $\mathbf{p}=\left(p_{1}, \ldots, p_{m}\right)$ with each $p_{i}>0$, recall from the first section that $\mu_{\mathbf{p}}$ is the self-similar measure supported on $E$ satisfying 


$$
\mu_{\mathbf{p}}=\sum_{i=1}^{m} p_{i} \mu_{\mathbf{p}} \circ f_{i}^{-1}
$$

In this section we will determine the local dimension of $\mu_{\mathbf{p}}$ at points in $\mathcal{U}_{k}(\Phi)$ and $\mathcal{U}_{\aleph_{0}}(\Phi)$.

\subsection{Local dimension of $\mu_{\mathbf{p}}$ at points in $\mathcal{U}_{k}(\Phi)$}

First we consider the local dimension of $\mu_{\mathbf{p}}$ at points in $\mathcal{U}_{k}(\Phi)$. Recall that $B(x, r)=$ $(x-r, x+r)$ is the open interval with center at $x$ and radius $r$.

Lemma 7.1. Let $h(x)=\alpha x+b$ with $\alpha>0$. Then

$$
h(B(c, r))=B(h(c), \alpha r) .
$$

Proof. For any $z=h(y) \in h(B(c, r))$ with $|y-c|<r$ we have

$$
|z-h(c)|=\alpha|y-c|<\alpha r
$$

which implies that $z \in B(h(c), \alpha r)$. On the other hand, for any $z \in B(h(c), \alpha r)$ we have

$$
\alpha\left|\frac{z-b}{\alpha}-c\right|=\left|h\left(\frac{z-b}{\alpha}\right)-h(c)\right|=|z-h(c)|<\alpha r .
$$

Thus, $z=h\left(\frac{z-b}{\alpha}\right)$ with $\frac{z-b}{\alpha} \in B(c, r)$.

In the following proposition we show that the local dimension of $\mu_{\mathbf{p}}$ at each point in $\mathcal{U}_{k}(\Phi)$ is uniquely determined by a point in $\mathcal{U}_{1}(\Phi)$.

Proposition 7.2. Let $\Phi=\left(E,\left\{f_{i}\right\}_{i=1}^{m}\right) \in \mathcal{E}$ and $k \geq 2$. Then for any $x \in \mathcal{U}_{k}(\Phi)$ there exists a word $\mathbf{i} \in\{1, \ldots, m\}^{*}$ and a unique $y \in \mathcal{U}_{1}(\Phi)$ such that $x=f_{\mathbf{i}}(y)$, and

$$
\underline{\operatorname{dim}}_{l o c} \mu_{\mathbf{p}}(x)=\underline{\operatorname{dim}}_{l o c} \mu_{\mathbf{p}}(y), \quad \overline{\operatorname{dim}}_{l o c} \mu_{\mathbf{p}}(x)=\overline{\operatorname{dim}}_{l o c} \mu_{\mathbf{p}}(y) .
$$

Proof. Since the proofs for different cases are similar, we assume without loss of generality that $f_{1}(I) \cap f_{2}(I) \neq \emptyset$ and $f_{m-1}(I) \cap f_{m}(I) \neq \emptyset$, where $I=\operatorname{conv}(E)$ is the convex hull of $E$. Let

$$
S=\bigcup_{i=1}^{m-1}\left(f_{i}(E) \cap f_{i+1}(E)\right)
$$

Then any $x \in S$ has at least two different codings. Define the expanding map on $E$ by

$$
T: E \rightarrow E ; \quad x \mapsto T(x)=\left\{\begin{array}{lll}
f_{i}^{-1}(x) & \text { if } & x \in f_{i}(E) \backslash S \\
f_{i}^{-1}(x) & \text { if } & x \in f_{i}(E) \cap f_{i+1}(E)
\end{array}\right.
$$


Then $\mathcal{U}_{1}(\Phi)=\left\{x \in E: T^{n}(x) \notin S \forall n \geq 0\right\}$.

Fix an integer $k \geq 2$ and take $x \in \mathcal{U}_{k}(\Phi)$. Then there exists a smallest integer $k_{1} \geq 0$ such that $y_{0}:=T^{k_{1}}(x) \in S$. So there exists a unique block $i_{1} \ldots i_{k_{1}} \in\{1, \ldots, m\}^{k_{1}}$ (it is the empty block $\epsilon$ if $\left.k_{1}=0\right)$ such that $x=f_{i_{1} \ldots i_{k_{1}}}\left(y_{0}\right)$. Since $y_{0} \in S$, there exists $j_{1} \in\{1, \ldots, m-1\}$ such that

$$
y_{0} \in f_{j_{1}}(E) \cap f_{j_{1}+1}(E)=f_{j_{1} m^{u}}(E)=f_{\left(j_{1}+1\right) 1^{v}}(E)
$$

where $u=u_{j_{1}}, v=v_{j_{1}} \in \mathbb{N}$ are the overlapping indices. Note that $x \notin \mathcal{U}_{\aleph_{0}}(\Phi)$. By Corollary 3.4 it follows that $y_{0} \notin\left\{f_{j_{1} m^{u}}(a), f_{\left(j_{1}+1\right) 1^{v}}(b)\right\}$. So, either there exists an integer $\ell_{1} \geq 0$ such that

$$
y_{0} \in f_{j_{1} m^{u} 1^{\ell_{1}}}(E) \backslash f_{j_{1} m^{u} 1^{\ell_{1}+1}}(E)
$$

or there exists an integer $\ell_{1}^{\prime} \geq 0$ such that

$$
y_{0} \in f_{\left(j_{1}+1\right) 1^{v} m^{\ell_{1}^{\prime}}}(E) \backslash f_{\left(j_{1}+1\right) 1^{v} m^{\ell_{1}^{\prime}+1}}(E) .
$$

Since the proof for the case in (7.2) can be handled similarly, without loss of generality we assume (7.1) holds. Note that $f_{m-1}(I) \cap f_{m}(I)=f_{(m-1) m^{p}}(I)=f_{m 1^{q}}(I)$ for some positive integers $p=u_{m-1}, q=v_{m-1}$. Then by using $f_{j m^{u}=f_{(j+1) 1^{v}}}$ and $f_{m 1^{q}}=f_{(m-1) m^{p}}$ it follows that

$$
f_{\left(j_{1}+1\right) 1^{v+\ell_{1}}}=f_{j_{1} m^{u} 1^{\ell_{1}}}=f_{j_{1} m^{u-1}(m-1) m^{p} 1^{\ell_{1}-q}}=\cdots=f_{j_{1} m^{u-1}\left((m-1) m^{p-1}\right)^{s} m 1^{\ell_{1}-s q}}
$$

for all $s=0,1, \ldots,\left\lfloor\frac{\ell_{1}}{q}\right\rfloor$. Here $\lfloor r\rfloor$ stands for the integer part of a real number $r$. So, by (7.1) there exists a unique $y_{1} \in E$ and $N_{1}:=\left\lfloor\frac{\ell_{1}}{q}\right\rfloor+2$ different blocks

$$
\begin{aligned}
& W_{1,1}:=\left(j_{1}+1\right) 1^{v+\ell_{1}}, \\
& W_{1,2}:=j_{1} m^{u} 1^{\ell_{1}}, \\
& W_{1,3}:=j_{1} m^{u-1}(m-1) m^{p 1^{\ell_{1}-q}} \\
& \quad \vdots \\
& W_{1, s+2}:=j_{1} m^{u-1}\left((m-1) m^{p-1}\right)^{s} m 1^{\ell_{1}-s q}, \\
& \quad \vdots \\
& W_{1, N_{1}}:=j_{1} m^{u-1}\left((m-1) m^{p-1}\right)^{N_{1}-2} m 1^{l_{1}-\left(N_{1}-2\right) q},
\end{aligned}
$$

such that

$$
y_{0}=f_{W_{1, i}}\left(y_{1}\right) \text { for all } i \in\left\{1, \ldots, N_{1}\right\}
$$


This implies that $y_{0}$ has $N_{1}$ different codings landing on $y_{1}$. Furthermore, note that $f_{1}(I) \cap f_{m}(I)=\emptyset$. One can verify that $y_{0}$ has precisely $N_{1}$ different codings landing on $y_{1}$. Observe that $x=f_{i_{1} \ldots i_{k_{1}}}\left(y_{0}\right)$ has a unique coding landing on $y_{0}$. Therefore, $x$ has precisely $N_{1}$ different codings landing on $y_{1}$.

If $y_{1} \in \mathcal{U}_{1}(\Phi)$, then $k=N_{1}$ and the proof is complete by taking $y=y_{1}$. Otherwise, there exists a smallest integer $k_{2} \geq 0$ such that $T^{k_{2}}\left(y_{1}\right) \in S$. By the same argument as above to the point $T^{k_{2}}\left(y_{1}\right)$ we can find a unique point $y_{2} \in E$ and $N_{2}$ different blocks $W_{2,1}, W_{2,2}, \ldots, W_{2, N_{2}}$ such that $f_{W_{2, i}}=f_{W_{2, j}}$ for any $i \neq j$, and $y_{1}=f_{W_{2, i}}\left(y_{2}\right)$. In other words, $y_{1}$ has precisely $N_{2}$ different codings landing on $y_{2}$. This, combined with the discussion from $x$ to $y_{1}$, implies that $x$ has precisely $M_{2} \leq N_{1} \cdot N_{2}$ different codings landing on $y_{2}$. We emphasize that $M_{2}$ might be strictly smaller than $N_{1} \cdot N_{2}$ if $y_{1} \in S$.

Since $k$ is finite, proceeding the above arguments for finitely many times we can find a unique point $y_{J} \in \mathcal{U}_{1}(\Phi)$ and $M_{J}$ different blocks $W_{J, 1}, W_{J, 2}, \ldots, W_{J, M_{J}}$ such that

$$
x=f_{W_{J, 1}}\left(y_{J}\right), \quad \text { and } \quad f_{W_{J, i}}=f_{W_{J, j}} \quad \forall i \neq j .
$$

Furthermore, $x$ has precisely $M_{J}$ different codings landing on $y_{J}$. Since $y_{J} \in \mathcal{U}_{1}(\Phi)$, this implies that $k=M_{J}$.

In the following it suffices to prove that the local dimension of $\mu_{\mathbf{p}}$ at $x$ is the same as that at $y_{J}$. Note that the contraction ratios of $f_{W_{J, i}}, 1 \leq i \leq M_{J}$ are the same, denote it by $r_{J}$. Define

$$
C_{J}:=\left\{i_{1} \ldots i_{n} \in\{1, \ldots, m\}^{*}: \prod_{\ell=1}^{n} r_{i_{\ell}} \leq r_{J}<\prod_{\ell=1}^{n-1} r_{i_{\ell}}\right\}
$$

Then $W_{J, i} \in C_{J}$ for all $i \in\left\{1, \ldots, M_{J}\right\}$, and

$$
\{1, \ldots, m\}^{\mathbb{N}}=\bigcup_{\mathbf{i} \in C_{J}}[\mathbf{i}]
$$

where the union is pairwise disjoint. Here $[\mathbf{i}]$ is a cylinder set generated by the block $\mathbf{i}$. So, for $r>0$

$$
\begin{aligned}
\mu_{\mathbf{p}}(B(x, r)) & =\sum_{W \in C_{J}} p_{W} \mu_{\mathbf{p}} \circ f_{W}^{-1}(B(x, r)) \\
& =\sum_{i=1}^{M_{J}} p_{W_{J, i}} \mu_{\mathbf{p}} \circ f_{W_{J, i}}^{-1}(B(x, r))+\sum_{W \in C_{J} \backslash\left\{W_{J, i}\right\}_{i=1}^{M_{J}}} p_{W} \mu_{\mathbf{p}} \circ f_{W}^{-1}(B(x, r)) .
\end{aligned}
$$

Observe that for any $W \in C_{J} \backslash\left\{W_{J, i}\right\}_{i=1}^{M_{J}}$ and for sufficiently small $r>0$ the open set $B(x, r)$ is separated from $f_{W}(E)$. So the second summation in (7.4) will disappear for small $r>0$. By (7.3), (7.4) and Lemma 7.1 it follows that for sufficiently small $r>0$, 


$$
\mu_{\mathbf{p}}(B(x, r))=\sum_{i=1}^{M_{J}} p_{W_{J, i}} \mu_{\mathbf{p}}\left(B\left(f_{W_{J, i}}^{-1}(x), r_{W_{J, i}}^{-1} r\right)\right)=\mu_{\mathbf{p}}\left(B\left(y_{J}, r_{J}^{-1} r\right)\right) \sum_{i=1}^{M_{J}} p_{W_{J, i}}
$$

Observe that $\sum_{i=1}^{M_{J}} p_{W_{J, i}}$ and $r_{J}^{-1}$ are both positive constants independent of $r$. This implies

$$
\underline{\operatorname{dim}}_{l o c} \mu_{\mathbf{p}}(x)=\underline{\operatorname{dim}}_{l o c} \mu_{\mathbf{p}}\left(y_{J}\right) \quad \text { and } \quad \overline{\operatorname{dim}}_{l o c} \mu_{\mathbf{p}}(x)=\overline{\operatorname{dim}}_{l o c} \mu_{\mathbf{p}}\left(y_{J}\right) .
$$

By Proposition 7.2 the local dimension of $\mu_{\mathbf{p}}$ at points in $\mathcal{U}_{k}(\Phi)$ is uniquely determined by the local dimension of $\mu_{\mathbf{p}}$ at points in $\mathcal{U}_{1}(\Phi)$. In the following result we explicitly determine the local dimension of $\mu_{\mathbf{p}}$ at points in $\mathcal{U}_{1}(\Phi)$.

Proposition 7.3. Let $\Phi=\left(E,\left\{f_{i}\right\}_{i=1}^{m}\right) \in \mathcal{E}$ with $f_{i}(x)=r_{i} x+b_{i}$ for $1 \leq i \leq m$. Then for any $y \in \mathcal{U}_{1}(\Phi)$ with its unique coding $\left(j_{k}\right)=j_{1} j_{2} \ldots \in\{1, \ldots, m\}^{\mathbb{N}}$ we have

$$
\underline{\operatorname{dim}}_{l o c} \mu_{\mathbf{p}}(y)=\liminf _{n \rightarrow \infty} \frac{\sum_{k=1}^{n} \log p_{j_{k}}}{\sum_{k=1}^{n} \log r_{j_{k}}}, \quad \overline{\operatorname{dim}}_{l o c} \mu_{\mathbf{p}}(y)=\limsup _{n \rightarrow \infty} \frac{\sum_{k=1}^{n} \log p_{j_{k}}}{\sum_{k=1}^{n} \log r_{j_{k}}} .
$$

Proof. Let $y \in \mathcal{U}_{1}(\Phi)$ with its unique coding $\left(j_{k}\right)$. Note by Condition (C) that there exists at least one pair of disjoint neighboring basic intervals, i.e., $f_{i}(I) \cap f_{i+1}(I)=\emptyset$ for some $i \in\{1, \ldots, m-1\}$. Let $I=[a, b]$ be the convex hull of $E$, and let

$$
g:=\min \left\{f_{i+1}(a)-f_{i}(b): f_{i}(I) \cap f_{i+1}(I)=\emptyset\right\} .
$$

Then $g>0$ is the length of the smallest gap between the neighboring basic intervals. Denote by $r_{\max }:=\max _{1 \leq i \leq m} r_{i}$. Then there exists a large integer $N_{1} \geq 1$ such that

$$
r_{\max }^{N_{1}}(b-a)<g
$$

Recall from Definition 1.2 that $\left(u_{1}, \ldots, u_{m}\right)$ and $\left(v_{1}, \ldots, v_{m}\right)$ are the overlapping vectors. Let

$$
N_{2}:=\max \left\{\max _{1 \leq i \leq m, u_{i} \neq \infty} u_{i}, \max _{1 \leq i \leq m, v_{i} \neq \infty} v_{i}\right\}
$$

Take $n>N_{2}$ sufficiently large, and let

$$
R_{n}:=r_{\max }^{N_{1}}(b-a) \prod_{k=1}^{n} r_{j_{k}}
$$

Clearly, the ball $B\left(y, R_{n}\right)$ contains the interval $f_{j_{1} \ldots j_{n+N_{1}}}(I)$. On the other hand, by (7.5) we have $R_{n}<g \prod_{k=1}^{n} r_{j_{k}}$. Observe that the basic intervals of higher level have similar geometrical structure to that of the first level. So, $B\left(y, R_{n}\right) \cap I$ is included in the basic interval $f_{j_{1} \ldots j_{n-N_{2}}}(I)$. 
Therefore,

$$
f_{j_{1} \ldots j_{n+N_{1}}}(I) \subset B\left(y, R_{n}\right) \cap I \subset f_{j_{1} \ldots j_{n-N_{2}}}(I),
$$

which implies

$$
\prod_{k=1}^{n+N_{1}} p_{j_{k}} \leq \mu_{\mathbf{p}}\left(B\left(y, R_{n}\right)\right) \leq \prod_{k=1}^{n-N_{2}} p_{j_{k}}
$$

Here we emphasize that the integers $N_{1}, N_{2}$ are independent of $n$. Taking the logarithms and dividing by $\log R_{n}$ on both sides of Equation (7.6) yields

$$
\frac{\sum_{k=1}^{n-N_{2}} \log p_{j_{k}}}{\log R_{n}} \leq \frac{\log \mu_{\mathbf{p}}\left(B\left(y, R_{n}\right)\right)}{\log R_{n}} \leq \frac{\sum_{k=1}^{n+N_{1}} \log p_{j_{k}}}{\log R_{n}}
$$

Denote by $p_{\text {min }}:=\min _{1 \leq i \leq m} p_{i}$ and $r_{\text {min }}:=\min _{1 \leq i \leq m} r_{i}$. Using the inequalities $p_{i} \geq$ $p_{\min }$ and $r_{\min } \leq r_{i} \leq r_{\max }$ in (7.7) it follows that

$$
\begin{aligned}
& \frac{\log \mu_{\mathbf{p}}\left(B\left(y, R_{n}\right)\right)}{\log R_{n}} \geq \frac{\sum_{k=1}^{n-N_{2}} \log p_{j_{k}}}{\sum_{k=1}^{n-N_{2}} \log r_{j_{k}}+N_{2} \log r_{\text {min }}+N_{1} \log r_{\text {max }}+\log (b-a)} \\
& \frac{\log \mu_{\mathbf{p}}\left(B\left(y, R_{n}\right)\right)}{\log R_{n}} \leq \frac{\sum_{k=1}^{n-N_{2}} \log p_{j_{k}}+\left(N_{1}+N_{2}\right) \log p_{\min }}{\sum_{k=1}^{n-N_{2}} \log r_{j_{k}}+\left(N_{1}+N_{2}\right) \log r_{\max }+\log (b-a)} .
\end{aligned}
$$

Letting $n \rightarrow \infty$ we obtain

$$
\begin{gathered}
\liminf _{n \rightarrow \infty} \frac{\log \mu_{\mathbf{p}}\left(B\left(y, R_{n}\right)\right)}{\log R_{n}}=\liminf _{n \rightarrow \infty} \frac{\sum_{k=1}^{n} \log p_{j_{k}}}{\sum_{k=1}^{n} \log r_{j_{k}}} \\
\limsup _{n \rightarrow \infty} \frac{\log \mu_{\mathbf{p}}\left(B\left(y, R_{n}\right)\right)}{\log R_{n}}=\limsup _{n \rightarrow \infty} \frac{\sum_{k=1}^{n} \log p_{j_{k}}}{\sum_{k=1}^{n} \log r_{j_{k}}} .
\end{gathered}
$$

Now for $R_{n+1}<r \leq R_{n}$ we have

$$
\frac{\log \mu_{\mathbf{p}}\left(B\left(y, R_{n}\right)\right)}{\log R_{n+1}} \leq \frac{\log \mu_{\mathbf{p}}(B(y, r))}{\log r} \leq \frac{\log \mu_{\mathbf{p}}\left(B\left(y, R_{n+1}\right)\right)}{\log R_{n}}
$$

Since $r_{\min } \leq \frac{R_{n+1}}{R_{n}} \leq r_{\max }$ and $R_{n} \rightarrow 0$ as $n \rightarrow \infty$, we have $\frac{\log R_{n+1}}{\log R_{n}} \rightarrow 1$ as $n \rightarrow \infty$. By (7.8) and (7.9) we conclude that

$\underline{\operatorname{dim}}_{l o c} \mu_{\mathbf{p}}(y)=\liminf _{r \rightarrow 0} \frac{\log \mu_{\mathbf{p}}(B(y, r))}{\log r}=\liminf _{n \rightarrow \infty} \frac{\log \mu_{\mathbf{p}}\left(B\left(y, R_{n}\right)\right)}{\log R_{n}}=\liminf _{n \rightarrow \infty} \frac{\sum_{k=1}^{n} \log p_{j_{k}}}{\sum_{k=1}^{n} \log r_{j_{k}}}$, and similarly,

$$
\overline{\operatorname{dim}}_{l o c} \mu_{\mathbf{p}}(y)=\limsup _{n \rightarrow \infty} \frac{\sum_{k=1}^{n} \log p_{j_{k}}}{\sum_{k=1}^{n} \log r_{j_{k}}}
$$




\subsection{Local dimension of $\mu_{\mathbf{p}}$ at points in $\mathcal{U}_{\aleph_{0}}(\Phi)$}

Recall from Proposition 3.4 that any $x \in \mathcal{U}_{\aleph_{0}}(\Phi)$ must be of the form

$$
x=f_{\mathbf{i}}\left(f_{1}(b)\right) \quad \text { if } \quad f_{1}(I) \cap f_{2}(I) \neq \emptyset
$$

for some $\mathbf{i} \in\{1, \ldots, m\}^{*}$, or of the form

$$
x=f_{\mathbf{j}}\left(f_{m}(a)\right) \quad \text { if } \quad f_{m-1}(I) \cap f_{m}(I) \neq \emptyset
$$

for some $\mathbf{j} \in\{1, \ldots, m\}^{*}$.

The following lemma can be shown by an argument similar to that in the proof of Proposition 7.2.

Lemma 7.4. Let $\Phi=\left(E,\left\{f_{i}\right\}_{i=1}^{m}\right) \in \mathcal{E}$ with the convex hull conv $(E)=[a, b]$.

- If $x=f_{\mathbf{i}}\left(f_{1}(b)\right) \in \mathcal{U}_{\aleph_{0}}(\Phi)$ with $\mathbf{i} \in\{1, \ldots, m\}^{*}$, then

$$
\operatorname{dim}_{l o c} \mu_{\mathbf{p}}(x)=\operatorname{dim}_{l o c} \mu_{\mathbf{p}}\left(f_{1}(b)\right) .
$$

- If $x=f_{\mathbf{j}}\left(f_{m}(b)\right) \in \mathcal{U}_{\aleph_{0}}(\Phi)$ with $\mathbf{j} \in\{1, \ldots, m\}^{*}$, then

$$
\operatorname{dim}_{l o c} \mu_{\mathbf{p}}(x)=\operatorname{dim}_{l o c} \mu_{\mathbf{p}}\left(f_{m}(b)\right)
$$

By Lemma 7.4 it suffices to consider the local dimension of $\mu_{\mathbf{p}}$ at $f_{1}(b)$ and $f_{m}(a)$.

Proposition 7.5. Let $\Phi=\left(E,\left\{f_{i}\right\}_{i=1}^{m}\right) \in \mathcal{E}$ with $I=\operatorname{conv}(E)=[a, b]$, and let $\mathbf{u}=$ $\left(u_{1}, \ldots, u_{m}\right), \mathbf{v}=\left(v_{1}, \ldots, v_{m}\right)$ be the overlapping vector.

(i) If $f_{1}(I) \cap f_{2}(I) \neq \emptyset$, then

$$
\operatorname{dim}_{l o c} \mu_{\mathbf{p}}\left(f_{1}(b)\right)=\min \left\{\frac{\log p_{m}}{\log r_{m}}, \frac{\log p_{2}+\left(v_{1}-1\right) \log p_{1}}{u_{1} \log r_{m}}\right\} .
$$

(ii) If $f_{m-1}(I) \cap f_{m}(I) \neq \emptyset$, then

$$
\operatorname{dim}_{l o c} \mu_{\mathbf{p}}\left(f_{m}(a)\right)=\min \left\{\frac{\log p_{1}}{\log r_{1}}, \frac{\log p_{m-1}+\left(u_{m-1}-1\right) \log p_{m}}{v_{m-1} \log r_{1}}\right\} .
$$

Proof. Since the proof of (ii) is similar, we only prove (i). Suppose $f_{1}(I) \cap f_{2}(I) \neq \emptyset$. Then there exist integers $u_{1}, v_{1} \geq 1$ such that $f_{1}(I) \cap f_{2}(I)=f_{1 m^{u_{1}}}(I)=f_{21^{v_{1}}}(I)$. We will determine the local dimension of $\mu_{\mathbf{p}}$ at $x=f_{1}(b) \in \mathcal{U}_{\aleph_{0}}(\Phi)$.

Denote by $\rho:=\min \left\{b-f_{m}(a), b-f_{m-1}(b)\right\}$. Then $\rho>0$, and the ball $B(b, \rho)$ has empty intersection with $f_{i}(I)$ for any $i \in\{1, \ldots, m-1\}$. This implies $B(b, \rho) \subset f_{m}(I)$. 
On the other hand, since the set sequence $f_{m^{n}}(I)$ decreases to $\{b\}$ as $n \rightarrow \infty$, there exists a large integer $N$ such that $B(b, \rho) \supset f_{m^{N}}(I)$. Therefore,

$$
f_{m^{N}}(I) \subset B(b, \rho) \subset f_{m}(I)
$$

Take a large integer $n$ such that $R_{n}:=r_{1} r_{m}^{n} \rho \in(0,1)$. Note that $x=f_{1}(b)=f_{1 m^{n}}(b)$. Then by Lemma 7.1 and the above inclusions it follows that

$$
f_{1 m^{n+N}}(I) \subset f_{1 m^{n}}(B(b, \rho))=B\left(x, R_{n}\right) \subset f_{1 m^{n+1}}(I) .
$$

Observe that $f_{1 m^{u_{1}}}=f_{21^{v_{1}}}$. Then for any $k \in \mathbb{N}_{\geq u_{1}}$,

$$
f_{1 m^{k}}(I)=f_{\left(21^{v_{1}-1}\right)^{s} 1 m^{k-s u_{1}}}(I) \quad \text { for all } s=0,1, \ldots,\left\lfloor\frac{k}{u_{1}}\right\rfloor .
$$

Applying (7.11) to (7.10) yields that for $n \geq u_{1}$

$$
\bigcup_{s=0}^{\left\lfloor\frac{n+N}{u_{1}}\right\rfloor} f_{\left(21^{v_{1}-1}\right)^{s} 1 m^{n+N-s u_{1}}}(I) \subset B\left(x, R_{n}\right) \subset \bigcup_{s=0}^{\left\lfloor\frac{n+1}{u_{1}}\right\rfloor} f_{\left(21^{v_{1}-1}\right)^{s} 1 m^{n+1-s u_{1}}}(I) .
$$

This implies

$$
\max _{0 \leq s \leq\left\lfloor\frac{n+N}{u_{1}}\right\rfloor}\left(p_{2} p_{1}^{v_{1}-1}\right)^{s} p_{1} p_{m}^{n+N-s u_{1}} \leq \mu_{\mathbf{p}}\left(B\left(x, R_{n}\right)\right) \leq \sum_{s=0}^{\left\lfloor\frac{n+1}{u_{1}}\right\rfloor}\left(p_{2} p_{1}^{v_{1}-1}\right)^{s} p_{1} p_{m}^{n+1-s u_{1}}
$$

Now we split the proof into the following two cases.

Case I. $p_{2} p_{1}^{v_{1}-1} \leq p_{m}^{u_{1}}$. Then taking the logarithms and dividing by $\log R_{n}$ on both sides of (7.12) it follows that

$$
\begin{aligned}
\frac{\log \mu_{\mathbf{p}}\left(B\left(x, R_{n}\right)\right)}{\log R_{n}} & \leq \frac{\log \left(\max _{0 \leq s \leq\left\lfloor\frac{n+N}{u_{1}}\right\rfloor} p_{1}\left(\frac{p_{2} p_{1}^{v_{1}-1}}{p_{m}^{u_{1}}}\right)^{s}\right)+(n+N) \log p_{m}}{n \log r_{m}+\log \left(r_{1} \rho\right)} \\
\frac{\log \mu_{\mathbf{p}}\left(B\left(x, R_{n}\right)\right)}{\log R_{n}} \geq & \geq \frac{\log \left(\sum_{s=0}^{\left\lfloor\frac{n+1}{u_{1}}\right\rfloor} p_{1}\left(\frac{p_{2} p_{1}^{v_{1}-1}}{p_{m}^{u_{1}}}\right)^{s}\right)+(n+1) \log p_{m}}{n \log r_{m}+\log \left(r_{1} \rho\right)} .
\end{aligned}
$$

Note that $p_{2} p_{1}^{v_{1}-1} \leq p_{m}^{u_{1}}$. Letting $n \rightarrow \infty$ in the above equation gives

$$
\lim _{n \rightarrow \infty} \frac{\log \mu_{\mathbf{p}}\left(B\left(x, R_{n}\right)\right)}{\log R_{n}}=\frac{\log p_{m}}{\log r_{m}}
$$


Case II. $p_{2} p_{1}^{v_{1}-1}>p_{m}^{u_{1}}$. Then the inequalities in (7.12) can be rearranged as

$$
\begin{aligned}
& \mu_{\mathbf{p}}\left(B\left(x, R_{n}\right)\right) \geq\left(p_{2} p_{1}^{v_{1}-1}\right)^{\frac{n+N}{u_{1}}} \max _{0 \leq s \leq\left\lfloor\frac{n+N}{u_{1}}\right\rfloor} p_{1}\left(\frac{p_{m}^{u_{1}}}{p_{2} p_{1}^{v_{1}-1}}\right)^{\frac{n+N-s u_{1}}{u_{1}}}, \\
& \mu_{\mathbf{p}}\left(B\left(x, R_{n}\right)\right) \leq\left(p_{2} p_{1}^{v_{1}-1}\right)^{\frac{n+1}{u_{1}}} \sum_{s=0}^{\left\lfloor\frac{n+1}{u_{1}}\right\rfloor} p_{1}\left(\frac{p_{m}^{u_{1}}}{p_{2} p_{1}^{v_{1}-1}}\right)^{\frac{n+1-s u_{1}}{u_{1}}} .
\end{aligned}
$$

By using $p_{2} p_{1}^{v_{1}-1}>p_{m}^{u_{1}}$ and an argument similar to the proof in Case I one can verify that

$$
\lim _{n \rightarrow \infty} \frac{\log \mu_{\mathbf{p}}\left(B\left(x, R_{n}\right)\right)}{\log R_{n}}=\frac{\log p_{2}+\left(v_{1}-1\right) \log p_{1}}{u_{1} \log r_{m}}
$$

By (7.13) and (7.14) it follows that

$$
\lim _{n \rightarrow \infty} \frac{\log \mu_{\mathbf{p}}\left(B\left(x, R_{n}\right)\right)}{\log R_{n}}=\min \left\{\frac{\log p_{m}}{\log r_{m}}, \frac{\log p_{2}+\left(v_{1}-1\right) \log p_{1}}{u_{1} \log r_{m}}\right\}
$$

Note that $R_{n+1} / R_{n}$ is bounded away from zero and infinity, and $R_{n} \rightarrow 0$ as $n \rightarrow \infty$. Therefore, we can conclude from the above equation that

$$
\operatorname{dim}_{l o c} \mu_{\mathbf{p}}(x)=\lim _{r \rightarrow 0} \frac{\log \mu_{\mathbf{p}}(B(x, r))}{\log r}=\min \left\{\frac{\log p_{m}}{\log r_{m}}, \frac{\log p_{2}+\left(v_{1}-1\right) \log p_{1}}{u_{1} \log r_{m}}\right\}
$$

Proof of Theorem 3. For the local dimension of $\mu_{\mathbf{p}}$ at points in $\mathcal{U}_{k}(\Phi)$ it follows from Propositions 7.2 and 7.3. And for the local dimension of $\mu_{\mathbf{p}}$ at points in $\mathcal{U}_{\aleph_{0}}(\Phi)$ it can be deduced from Lemma 7.4 and Proposition 7.5.

\section{Final remarks}

We believe some of the results obtained in this paper can be extended to a much more general class of SIFS (see e.g., [18]). Observe that Condition (D) in our class $\mathcal{E}$, also called the complete overlap condition, is very strong. For a possible extension one might think of dropping out this complete overlap condition.

Example 8.1. Let $E$ be the attractor of the IFS $\left\{f_{i}(x)\right\}_{i=1}^{m}$ with $m \geq 5$. Denote the convex hull of $E$ by $I=[a, b]$. Suppose $\Phi=\left(E,\left\{f_{i}\right\}_{i=1}^{m}\right)$ satisfies the following conditions.

- $f_{1 m}=f_{21}$;

- $f_{2}(I) \cap f_{i}(I)=\emptyset$ for any $i>2$;

- $f_{m} \cap f_{j}(I)=\emptyset$ for any $j<m$;

- $f_{i}(I) \subset\left(f_{2}(b), f_{m}(a)\right)$ for any $i \in\{3, \ldots, m-1\}$. 
Observe by the last condition that we have a lot of flexibility for the locations of $f_{i}(I)$ for $3 \leq i \leq m-1$. Then $\Phi$ does not necessarily belong to $\mathcal{E}$. But one can still show that $\operatorname{dim}_{H} \mathcal{U}_{k}(\Phi)=\operatorname{dim}_{H} \mathcal{U}_{1}(\Phi)$ for any $k \in \mathbb{N}$.

The object studied in this paper is in one dimension. It would be interesting to consider a higher dimensional analogue.

\section{Acknowledgments}

The authors wish to thank the anonymous referee for many useful comments, which greatly improved the presentation of the paper. They also thank Junjie Miao for many discussions on the earlier version of Theorem 3. Jiang was supported by NSFC No. 11701302, Zhejiang Provincial Natural Science Foundation of China with No. LY20A010009, and the K.C. Wong Magna Fund in Ningbo University. Kong was supported by NSFC No. 11971079 and the Fundamental and Frontier Research Project of Chongqing No. cstc2019jcyj-msxmX0338 and No. cx2019067. Li was supported by NSFC No. 11671147, 11571144 and Science and Technology Commission of Shanghai Municipality (STCSM) No. 18dz2271000. Xi was supported by NSFC No. 11831007.

\section{References}

[1] R. Alcaraz Barrera, S. Baker, D. Kong, Entropy, topological transitivity, and dimensional properties of unique q-expansions, Trans. Am. Math. Soc. 371 (5) (2019) 3209-3258.

[2] S. Baker, On small bases which admit countably many expansions, J. Number Theory 147 (2015) $515-532$.

[3] K. Dajani, M. de Vries, Invariant densities for random $\beta$-expansions, J. Eur. Math. Soc. 9 (1) (2007) $157-176$.

[4] K. Dajani, K. Jiang, D. Kong, W. Li, Multiple expansions of real numbers with digits set $\{0,1, q\}$, Math. Z. 291 (3-4) (2019) 1605-1619.

[5] K. Dajani, D. Kong, Y. Yao, On the structure of $\lambda$-cantor set with overlaps, Adv. Appl. Math. 108 (2019) 97-125.

[6] M. de Vries, V. Komornik, Unique expansions of real numbers, Adv. Math. 221 (2) (2009) 390-427.

[7] M. de Vries, V. Komornik, Expansions in non-integer bases, in: Combinatorics, Words and Symbolic Dynamics, in: Encyclopedia Math. Appl., vol. 159, Cambridge Univ. Press, Cambridge, 2016, pp. $18-58$.

[8] Q. Deng, J. Harding, T. Hu, Hausdorff dimension of self-similar sets with overlaps, Sci. China Ser. A 52 (1) (2009) 119-128.

[9] R. Durrett, Probability: Theory and Examples, fourth edition, Cambridge Series in Statistical and Probabilistic Mathematics, vol. 31, Cambridge University Press, Cambridge, 2010.

[10] P. Erdős, I. Joó, V. Komornik, Characterization of the unique expansions $1=\sum_{i=1}^{\infty} q^{-n_{i}}$ and related problems, Bull. Soc. Math. Fr. 118 (1990) 377-390.

[11] P. Erdős, M. Horváth, I. Joó, On the uniqueness of the expansions $1=\sum q^{-n_{i}}$, Acta Math. Hung. 58 (3-4) (1991) 333-342.

[12] P. Erdős, I. Joó, On the number of expansions $1=\sum q^{-n_{i}}$, Ann. Univ. Sci. Bp. Eötvös, Sect. Math. 35 (1992) 129-132.

[13] K. Falconer, Fractal Geometry: Mathematical Foundations and Applications, third edition, John Wiley \& Sons, Ltd., Chichester, 2014.

[14] D.-J. Feng, K.-S. Lau, Multifractal formalism for self-similar measures with weak separation condition, J. Math. Pures Appl. (9) 92 (4) (2009) 407-428.

[15] P. Glendinning, N. Sidorov, Unique representations of real numbers in non-integer bases, Math. Res. Lett. 8 (2001) 535-543. 
[16] M. Hochman, On self-similar sets with overlaps and inverse theorems for entropy, Ann. Math. 180 (2) (2014) 773-822.

[17] J.E. Hutchinson, Fractals and self-similarity, Indiana Univ. Math. J. 30 (5) (1981) 713-747.

[18] K. Jiang, K. Dajani, Subshifts of finite type and self-similar sets, Nonlinearity 30 (2) (2017) 659-686.

[19] R. Kenyon, Projecting the one-dimensional Sierpinski gasket, Isr. J. Math. 97 (1997) 221-238.

[20] V. Komornik, Expansions in noninteger bases, Integers 11B:Paper No. A9 (2011) 30.

[21] V. Komornik, D. Kong, Bases in which some numbers have exactly two expansions, J. Number Theory 195 (2019) 226-268.

[22] V. Komornik, D. Kong, W. Li, Hausdorff dimension of univoque sets and Devil's staircase, Adv. Math. 305 (2017) 165-196.

[23] K.-S. Lau, S.-M. Ngai, Multifractal measures and a weak separation condition, Adv. Math. 141 (1) (1999) 45-96.

[24] D. Lind, B. Marcus, An Introduction to Symbolic Dynamics and Coding, Cambridge University Press, Cambridge, 1995.

[25] R.D. Mauldin, S.C. Williams, Hausdorff dimension in graph directed constructions, Trans. Am. Math. Soc. 309 (2) (1988) 811-829.

[26] S.M. Ngai, Y. Wang, Hausdorff dimension of self-similar sets with overlaps, J. Lond. Math. Soc. 63 (2001) 655-672.

[27] S.-M. Ngai, Y. Xie, $L^{q}$-spectrum of self-similar measures with overlaps in the absence of second-order identities, J. Aust. Math. Soc. 106 (1) (2019) 56-103.

[28] W. Parry, On the $\beta$-expansions of real numbers, Acta Math. Acad. Sci. Hung. 11 (1960) 401-416.

[29] H. Rao, Z.-Y. Wen, A class of self-similar fractals with overlap structure, Adv. Appl. Math. 20 (1) (1998) 50-72.

[30] A. Rényi, Representations for real numbers and their ergodic properties, Acta Math. Acad. Sci. Hung. 8 (1957) 477-493.

[31] N. Sidorov, Almost every number has a continuum of $\beta$-expansions, Am. Math. Mon. 110 (9) (2003) 838-842.

[32] N. Sidorov, Expansions in non-integer bases: lower, middle and top orders, J. Number Theory 129 (4) (2009) $741-754$.

[33] Y. Zou, J. Lu, W. Li, Unique expansion of points of a class of self-similar sets with overlaps, Mathematika 58 (2) (2012) 371-388. 